\title{
UM MODELO DE REGRESSÃO BETA: TEORIA E APLICAÇÕES
}

\author{
Marcos Santos de Oliveira
}

\author{
Dissertação Apresentada ho \\ Instituto de MATEmática e Estatístea \\ da Universidade de Sĩo Pálo

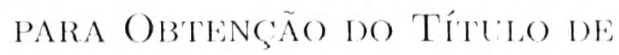 \\ Mestre EM ESTATÍSTICA
}

Área de Concentração: Estatística
Orientadora: Profa. Dra. Silvia Lopes de Paula Ferrari

Durante a elaboraça deste trabalho o autor

receber apoto financeiro do CNPq 


\title{
UM MODELO DE REGRESSÃO BETA: TEORIA E APLICAÇÕES
}

\author{
Este exemplar corresponde à redação final da \\ dissertação de mestrado devidamente corrigida \\ e defendida por Marcos Santos de Oliveira \\ e aprovada pela comissão julgadora.
}

São Paulo, 12 de abril de 2004.

Banca examinadora:

- Profa. Dra. Silvia Lopes de Paula Ferrari (Orientadora) - IME - USP

- Prof. Dr. Gilberto Alvarenga Paula - IME - USP

- Profa. Dra. Clarice Garcia Borges Demétrio - ESALQ - USP 
Aos meus pais, que com muito

sacrificio e persistência

colocaram-me no caminho

do saber.

A Daniela Carine Ramires, que me ajudou a acreditar que os limites individuais são apenas obstáculos a serem superados continuamente em nossa vida.

A todos os meus amigos, que nas horas críticas me impulsionaram e me ensinaram que o amanhã se conquista hoje. 


\section{Agradecimentos}

- Primeiramente a Deus, por permitir que eu conquistasse esta etapa.

- Ao meu pai, Albertino, e à minha querida mãe, Maria Aparecida, por todo o incentivo que sempre me deram, sem o qual eu não teria conseguido concluir esta etapa tão importante.

- Um agradecimento muito especial à Daniela Carine Ramires, pelo amor, carinho e constante apoio nas horas mais difíceis.

- Aos meus familiares, que mesmo à distância, sempre me incentivaram.

- Ao amigo e eterno Professor, Haroldo Santos Nogueira, por ter me descoberto na infância e me incentivado, em todos os sentidos, até os dias atuais.

- À Profa. Dra. Vilma Mayumi Tachibana, que durante a graduação me orientou com brilhantismo em projetos de iniciação científica e para a vida.

- Aos professores do Departamento de Estatística do IME - USP, por suas contribuições à minha formação como Estatístico.

- A todos os amigos e colegas do IME - USP, em especial ao amigo Cléber da Costa Figueiredo, pela amizade, apoio e convívio durante esses dois anos.

- Finalmente, aos participantes da banca examinadora pelas valiosas contribuições. 


\section{Agradecimento Especial}

À Silvia Lopes de Paula Ferrari, Professora Doutora do Departamento de Estatística do Instituto de Matemática e Estatística da Universidade de São Paulo USP, que, dotada de apurada sensibilidade no trato com seus semelhantes, tem possibilitado o desenvolvimento daqueles que a cercam.

Ao $\mathrm{CNPq}$, pelos recursos financeiros disponibilizados desde o início dos meus estudos de pós-graduação, sem os quais este trabalho não seria concretizado.

“... um discípulo nunca pode imitar os passos de seu guia, pois cada um tem a sua maneira de ver a vida, de conviver com as dificuldades e com as conquistas. Ensinar é mostrar que é possivel. Aprender é tornar a si mesmo".

"Vejo as coisas como são e me pergunto por que? Sonho as coisas como as quero e me pergunto por que não?" 


\section{Resumo}

Esta dissertação compreende o estudo de um modelo de regressão adequado para situações em que a variável resposta é medida de forma contínua no intervalo unitário como, por exemplo, dados de taxas ou proporções. O trabalho foi baseado no artigo de Ferrari e Cribari-Neto (2004) que propõe um modelo de regressão em que a resposta tem distribuição beta utilizando uma parametrização da lei beta que é indexada pela média e um parâmetro de dispersão. Nossa contribuição está, primeiramente, na avaliação, via simulação, da qualidade das aproximações utilizadas nas inferências sobre os parâmetros em amostras finitas. Em particular, avaliamos o viés do estimador de máxima verossimilhança, a proximidade de sua distribuição à distribuição normal de referència, a distorção do tamanho dos testes de razão de verossimilhanças, escore e Wald e a qualidade da aproximação das distribuições das estatísticas dos testes por uma distribuição qui-quadrado adequada. Desenvolvemos também uma análise e discussão de duas novas aplicações do modelo de regressão beta a conjuntos de dados reais. 


\section{Abstract}

This dissertation deals with the study of a regression model that is tailored for situations where the response variable is measured continuously on the standard unit interval, such as rates or proportions. We follow the work by Ferrari and Cribari-Neto (2004) who proposed a regression model where the response is beta distributed using a parameterization of the beta law that is indexed by mean and dispersion parameters. Our contribution is the evaluation, by simulation, of the goodness of the approximations that are used in statistical inference in finite samples. In particular, we evaluate the bias of the maximum likelihood estimator, the agreement of the distribution of this estimator and the reference normal distribution, the size distortion of the usual likelihood ratio, score and Wald tests and the goodness of the approximation of the distribution of the test statistics by the appropriate chi-squared distribution. Finally, two new practical applications that employ real data are presented and discussed. 


\section{Índice}

1 Introdução 1

1.1 Organização da Dissertação . . . . . . . . . . . . . . 2

2 Distribuição Beta 4

2.1 Introdução . . . . . . . . . . . . . . . . . . . 4

2.2 Definição e Propriedades ... . . . . . . . . . . . . . . 4

2.3 Estimação dos Parâmetros . . . . . . . . . . . . . . . . . . 9

2.3.1 Método de Máxima Verossimilhança . . . . . . . . . . . 9

2.3.2 Método dos Momentos ... . . . . . . . . . . . 11

2.3 .3 Eficiencia Assintótica . . . . . . . . . . . . . . . . 13

2.4 Exemplos ............................ 16

2.4 .1 Oxidação de Amônia . . . . . . . . . . . . . . . 16

2.4.2 Contaminação de Amendoim . . . . . . . . . . . . . 18 
Indice

3 Modelo de Regressão Beta 21

3.1 Introdução . . . . . . . . . . . . . . . . . . . . 21

3.2 Definição. . . . . . . . . . . . . . . . . . . . . . 22

3.3 Funções de Ligação . . . . . . . . . . . . . . . . . . . . . . . . . . . . 24

3.4 Função Escore e Matriz de Informação . . . . . . . . . . . . . . . 26

3.5 Estimação dos Parâmetros . . . . . . . . . . . . . . . . . . . . 31

3.6 Testes de Hipóteses . . . . . . . . . . . . . . . . . . . . . . . 34

3.6.1 Teste da Razão de Verossimilhanças . . . . . . . . . . . . . . 34

3.6 .2 Teste Escore . . . . . . . . . . . . . . . . . 35

3.6 .3 Teste de Wald . . . . . . . . . . . . . . . . . . . 35

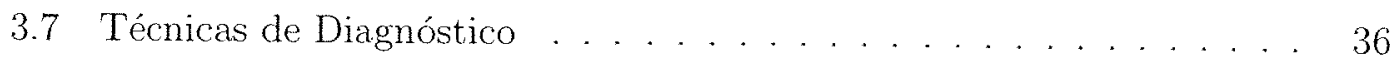

3.7 .1 Resíduo Componente do Desvio . . . . . . . . . . . . 38

3.7.2 Gráficos de Probabilidade Meio-Normal com Envelopes . . . . 39

3.7 .3 Leverage Generalizado . . . . . . . . . . . . . . . 40

3.7 .4 Influência . . . . . . . . . . . . . . . . 44

4 Avaliação de Resultados Assintóticos em Amostras Finitas 45

4.1 Introdução . . . . . . . . . . . . . . . . . . . . . . 45

4.2 Detalhes Metodológicos . . . . . . . . . . . . . . . . . 46

4.3 Comportamento dos Estimadores . . . . . . . . . . . . 47

4.4 Tamanho dos Testes ..................... 54 
Indice

5 Aplicações 63

5.1 Oxidação de Amônia . . . . . . . . . . . . . . . . . . 63

5.2 Contaminação de Amendoim . . . . . . . . . . . . . . . . 69

$\begin{array}{lll}6 & \text { Considerações Finais } & 78\end{array}$

A Informação de Fisher para $(p, q) \quad 80$

B Matriz de Covariâncias Assintóticas para os EMM 82

$\begin{array}{ll}\text { C Programas } & 85\end{array}$

$\begin{array}{ll}\text { Referências Bibliográficas } & 96\end{array}$ 


\section{Capítulo 1}

\section{Introdução}

Em muitas situações práticas, o interesse é analisar o comportamento de uma variável em função de outras. Nesses casos, os pesquisadores normalmente utilizam a teoria de modelos de regressão para identificar as possíveis relações entre as variáveis. Em particular, o modelo de regressão linear é geralmente utilizado nas aplicações. No entanto, ele não é apropriado para situações em que a resposta é restrita ao intervalo unitário $(0,1)$, pois pode ajustar valores para a variável de interesse que exceda o limite inferior ou superior desse intervalo. Uma possível solução é transformar a variável dependente de tal forma que esta assuma valores em toda a reta e então, modelar a média da resposta transformada como um preditor linear baseado em um conjunto de variáveis explicativas. No entanto, esse enfoque apresenta algumas desvantagens, sendo uma delas o fato de que os parâmetros do modelo não podem ser facilmente interpretados em termos da resposta original.

Ferrari e Cribari-Neto (2004) propuseram um modelo de regressāo para situações em que a variável dependente $(y)$ é medida de forma contínua no intervalo unitário padrão, isto é, $0<y<1$. O modelo proposto é baseado na suposição que a resposta tem distribuição beta utilizando uma parametrização da lei beta que é indexada pela média e um parâmetro de dispersão. Como se sabe, a distribuição beta é muito 
flexível para modelar proporções, pois sua densidade pode assumir diferentes formas dependendo dos valores dos dois parâmetros que indexam a distribuição. Johnson, Kotz e Balakrishnam (1995, p. 235) relatam que a distribuição beta é muito versátil e uma variedade de incertezas podem ser modeladas por ela, sendo que sua flexibilidade encoraja seu uso empírico nas aplicações. Algumas aplicações da distribuição beta são discutidas por Bury (1999) e por Johnson, Kotz e Balakrishnam (1995). Contudo, essas aplicações não envolvem situações em que o investigador necessita impor uma estrutura de regressão para a variável resposta. Em nosso trabalho, o interesse está em situações em que o comportamento das respostas possa ser modelado como uma função de um conjunto de variáveis explicativas.

O principal objetivo deste trabalho é discutir o modelo de regressão beta no que diz respeito a sua definição, resultados de inferência e aplicaçōes. Adicionalmente, apresentamos um estudo de simulação que procurou avaliar a qualidade das aproximações utilizadas nas inferências sobre os parâmetros em amostras finitas.

\subsection{Organização da Dissertação}

A presente dissertação de mestrado está dividida em seis capítulos. No segundo capítulo, apresentamos um estudo sobre a distribuição beta no que diz respeito a sua definição, propriedades e inferências sobre seus parâmetros. Além disso, são apresentados os resultados assintóticos acerca dos estimadores de máxima verossimilhança e do método dos momentos, tais como normalidade, consistência e eficiência.

No terceiro capítulo, apresentamos o modelo de regressão beta proposto por Ferrari e Cribari-Neto (2004), algumas funções de ligação, a função escore e matriz de informação, a estimação dos parâmetros de regressão, testes de hipóteses, intervalos 
de confiança e algumas medidas de diagnóstico.

No quarto capítulo, avaliamos numericamente a qualidade das aproximações utilizadas nas inferências sobre os parâmetros do modelo de regressão beta em amostras finitas. Nesse capítulo, o interesse é avaliar o viés do estimador de máxima verossimilhança, a proximidade de sua distribuição à distribuição normal de referência, a distorção de tamanho dos testes da razão de verossimilhanças, escore e Wald e também verificar a qualidade da aproximação das distribuiçōes das estatísticas dos testes por uma distribuição qui-quadrado adequada.

No quinto capítulo, analisamos dois conjuntos de dados reais utilizando o modelo de regressão beta. A primeira aplicação refere-se a um estudo químico sobre oxidação de amônia e a segunda é da área de agricultura, um estudo sobre contaminação de amendoins.

Por fim, no sexto capítulo apresentamos conclusões de todos os resultados obtidos em cada capítulo. 


\section{Distribuição Beta}

\subsection{Introdução}

Este capítulo apresenta um estudo sobre a distribuição beta no que diz respeito a sua definição, propriedades, inferências sobre seus parâmetros e aplicações. As principais referências bibliográficas utilizadas para este capítulo foram os livros de Bury (1999, Capítulo 14) e Johnson, Kotz e Balakrishnan (1995, Capítulo 25). Além disso, são apresentados os resultados assintóticos acerca dos estimadores de máxima verossimilhança e do método dos momentos, tais como normalidade, consistência e eficiência.

\subsection{Definição e Propriedades}

A família de distribuições beta é composta de todas as distribuições com função densidade de probabilidade (f.d.p.) da forma

$$
f(y ; p, q)=\frac{\Gamma(p+q)}{\Gamma(p) \Gamma(q)} y^{p-1}(1-y)^{q-1}, \quad 0<y<1, \quad p>0, \quad q>0,
$$


em que $\Gamma(p)$ é a função gama avaliada no ponto $p$, ou seja,

$$
\Gamma(p)=\int_{0}^{\infty} y^{p-1} e^{-y} d y
$$

A f.d.p. em (2.1) é a forma padrão da distribuição beta com parâmetros $p$ e $q$ e é a forma que será usada na maior parte deste capítulo. Note que a distribuição uniforme é um caso particular de (2.1) quando $p=q=1$. Esta distribuição é um modelo estatístico importante para variáveis aleatórias que têm seus valores restritos ao intervalo unitário.

Este modelơ não apresenta a estrutura dos modelos de locação-escala, pois ambos $p$ e $q$ são parâmetros de forma. Um fato interessante da distribuição beta é que

$$
y \sim \operatorname{Beta}(p, q) \quad \Rightarrow \quad 1-y \sim \operatorname{Beta}(q, p) .
$$

Além disso,

$$
y_{1} \sim \operatorname{Gama}(p, \beta) \text { e } y_{2} \sim \operatorname{Gama}(q, \beta), y_{1} \text { e } y_{2} \text { ind. } \Rightarrow \frac{y_{1}}{y_{1}+y_{2}} \sim \operatorname{Beta}(p, q) .
$$

A função de distribuição acumulada (f.d.a.) de uma variável aleatória (v.a.) com distribuição $\operatorname{Beta}(a, b)$ é dada por

$$
F(y ; p, q)=\frac{\Gamma(p+q)}{\Gamma(p) \Gamma(q)} \int_{0}^{y} z^{p-1}(1-z)^{q-1} d z
$$

A inversa da razão de funções gamas na expressão (2.3),

$$
B(p, q)=\frac{\Gamma(p) \Gamma(q)}{\Gamma(p+q)}
$$

é chamada função beta e a integral em (2.3),

$$
B_{y}(p, q)=\int_{0}^{y} z^{p-1}(1-z)^{q-1} d z
$$

é denominada função beta incompleta. Note que $B_{y}(p, q) \rightarrow B(p, q)$, quando $y \rightarrow 1$. A f.d.a. de y pode assim ser expressa como a razão da função beta incompleta pela 
função beta, ou seja,

$$
F(y ; p, q)=\frac{B_{y}(p, q)}{B(p, q)}
$$

e de (2.2) segue que

$$
F(y ; p, q)=F(1-y ; q, p)
$$

Existem tabelas disponíveis para a função beta (ver Pearson e Johnson, 1968). No entanto, essas funções são facilmente calculadas numericamente. Quando $p$ e $q$ são números inteiros, a f.d.a. da distribuição beta pode ser avaliada usando a identidade

$$
F(y ; p, q)=1-\sum_{t=0}^{p-1}\left(\begin{array}{c}
p+q-1 \\
t
\end{array}\right) y^{t}(1-y)^{p+q-1-t} .
$$

O r-ésimo momento populacional de $y$ em relação à origem é dado por

$$
\begin{aligned}
\mu_{r}^{\prime}(y) & =\frac{B(p+r, q)}{B(p, q)}=\frac{\Gamma(p+r) \Gamma(p+q)}{\Gamma(p) \Gamma(p+q+r)} \\
& =\frac{p^{[r]}}{(p+q)^{[r]}} \quad \text { (se } r \text { é um valor inteiro) }
\end{aligned}
$$

em que $x^{[r]}=x(x+1) \cdots(x+r-1)$ é o fatorial crescente. Em particular, o valor esperado da variável aleatória y é dado por

$$
\mu_{1}^{\prime}(y)=\mathrm{E}(y)=\frac{p}{p+q},
$$

e sua variância é

$$
\mu_{2}(y)=\operatorname{Var}(y)=\frac{p q}{(p+q)^{2}(p+q+1)} .
$$

Pham-Gia (1994) estabeleceu alguns limites para Var(y). Especificamente, ele mostrou que $\operatorname{Var}(y)<1 / 4$, e se a densidade de $y$ é unimodal (isto é, $p>1$ e $q>1$ ) $\operatorname{Var}(y)<1 / 12$; adicionalmente, se a densidade de $y$ é de formato $U$ (isto é, $p<1 \mathrm{e}$ $q<1$ ), ele provou que $1 / 12<\operatorname{Var}(y)<1 / 4$. 
O coeficiente de variação de $y$ é dado por

$$
\operatorname{cv}(y)=\frac{\sqrt{\operatorname{Var}(y)}}{\mathrm{E}(y)}=\sqrt{\frac{q}{p(p+q+1)}} .
$$

Essa medida caracteriza a dispersão da distribuição de $y$ em termos relativos à sua média.

A distribuição beta possui um valor modal quando $p+q>2$ e $p \geq 1$ dado por

$$
y_{m}=\frac{p-1}{p+q-2} .
$$

O coeficiente de assimetria é dado por

$$
\gamma_{1}=\frac{2(q-p)}{p+q+2} \sqrt{\frac{p+q+1}{p q}} .
$$

Esta medida caracteriza como e quanto a distribuição se afasta da condição de simetria. Se $p=q$, então $\gamma_{1}=0$ e a f.d.p. é simétrica. Se $q>p$ então $\gamma_{1}>0$ e a f.d.p. é assimétrica à direita. Similarmente, $q<p$ fornece $\gamma_{1}<0$ para uma distribuição assimétrica à esquerda.

O coeficiente de curtose é dado por

$$
\gamma_{2}=\frac{3(p+q+1)\left[2(p+q)^{2}+p q(p+q-6)\right]}{p q(p+q+2)(p+q+3)}
$$

e é uma medida que procura caracterizar o formato da distribuição quanto ao seu achatamento, sendo menor do que 3 para as distribuições platicúrticas, igual a 3 para uma distribuição mesocúrtica e maior do que 3 para as distribuições leptocúrticas. Mais detalhes sobre formatos de distribuições ver Costa Neto (1985, pg. 32). Segundo Bury (1999), para o caso simétrico $(p=q=\lambda), \gamma_{2} \rightarrow 3$ quando $\lambda$ cresce, fazendo a função densidade da distribuição beta se aproximar da distribuição normal com média $1 / 2$ e variância $1 /[4(2 \lambda+1)]$.

Como a distribuição beta é caracterizada por dois parâmetros de forma, existe muita flexibilidade no formato da densidade. A Figura 2.1 ilustra esta flexibilidade 

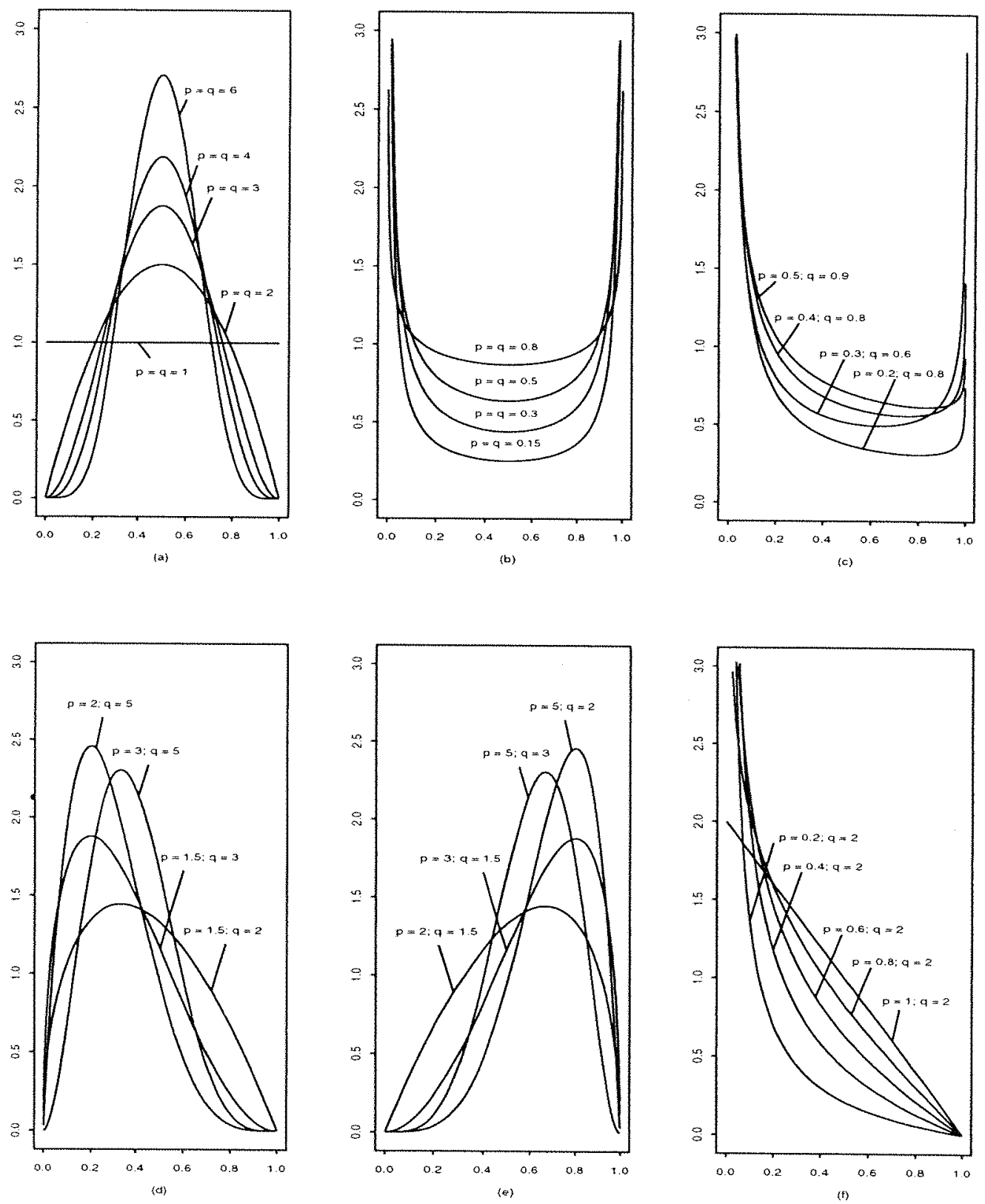

Figura 2.1: Densidades beta para diferentes combinaçöes de $(p, q)$.

para várias combinações de valores dos parâmetros $p$ e $q$. As Figuras 2.1 (a) e (b) apresentam as funções densidades de probabilidades que são simétricas $(p=q)$, sendo unimodal em (a) e de formato $U(p, q<1)$ em (b). As Figuras 2.1 (c), (d) e (e) 
apresentam funções densidades de probabilidades assimétricas $(p \neq q)$. O formato $U$ é caracterizado novamente em (c), enquanto que em (d) e (e) temos a representação unimodal de assimetria à direita e à esquerda, respectivamente. Finalmente, na Figura 2.1 (f) temos a densidade de formato $J$; aqui $(p-1)(q-1)<0$.

\subsection{Estimação dos Parâmetros}

Nesta seção discutimos a estimação dos parâmetros para a distribuição beta através de dois métodos clássicos. O primeiro é o método de máxima verossimilhança em que os estimadores são obtidos a partir da maximização da função de verossimilhança. O segundo método considerado é o método dos momentos em que os estimadores são obtidos igualando-se os momentos amostrais aos correspondentes momentos populacionais. Adicionalmente, são apresentados os resultados de convergências para os estimadores de máxima verossimilhança e dos momentos, juntamente com uma análise da eficiência relativa assintótica para estes estimadores.

\subsubsection{Método de Máxima Verossimilhança}

Sejam $y_{1}, \ldots, y_{n}$ uma amostra aleatória de tamanho $n$ da variável aleatória $y$ com função densidade (ou de probabilidade) beta definida em $(2.1) \operatorname{com} \theta=(p, q) \in$ $\Theta=\mathbb{R}^{+} \times \mathbb{R}^{+}$, sendo $\Theta$ o espaço paramétrico. A função de verossimilhança de $\theta$ correspondente à amostra observada $y_{1}, \ldots, y_{n}$ é dada por

$$
L(\theta)=L(p, q)=\prod_{t=1}^{n} \frac{y_{t}^{p-1}\left(1-y_{t}\right)^{q-1}}{B(p, q)}=[B(p, q)]^{-n} \prod_{t=1}^{n} y_{t}^{p-1} \prod_{t=1}^{n}\left(1-y_{t}\right)^{q-1}
$$


em que $B(p, q)$ é a função beta definida em (2.4). Aplicando o logaritmo natural em ambos os lados da equação (2.8) tem-se

$\ell(p, q)=\log L(p, q)=-n \cdot \log B(p, q)+(p-1) \sum_{t=1}^{n} \log y_{t}+(q-1) \sum_{t=1}^{n} \log \left(1-y_{t}\right)$

As equações de máxima verossimilhança são obtidas derivando-se $\ell(p, q)$ em relação a cada parâmetro e igualando-se o resultado a zero. Assim, temos as seguintes equações:

$$
\psi(\widehat{p})-\psi(\widehat{p}+\widehat{q})=\frac{1}{n} \sum_{t=1}^{n} \log y_{t} \quad \text { e } \psi(\widehat{q})-\psi(\widehat{p}+\widehat{q})=\frac{1}{n} \sum_{t=1}^{n} \log \left(1-y_{t}\right),
$$

em que $\psi(\hat{\lambda})$ é a função digama definida como

$$
\psi(\lambda)=\frac{\mathrm{d} \log \Gamma(\lambda)}{\mathrm{d} \lambda}=\frac{\Gamma^{\prime}(\lambda)}{\Gamma(\lambda)}, \quad \lambda>0
$$

Soluções analíticas explícitas das equações de máxima verossimilhança não podem ser obtidas para os parâmetros da distribuição beta. Neste caso, os estimadores de máxima verossimilhança são facilmente obtidos por meio de métodos numéricos de maximização, como por exemplo, o método escore de Fisher (ver Cordeiro, 1992, Seção 1.4). Para $\widehat{p}$ e $\widehat{q}$ grandes, as expressões dadas em (2.9) podem ser aproximadas via $\psi(y) \doteq \log \left(y-\frac{1}{2}\right)$, ou seja,

$$
\log \frac{\widehat{p}-\frac{1}{2}}{\widehat{p}+\widehat{q}-\frac{1}{2}} \doteq \log \prod_{t=1}^{n} y_{t}^{1 / n} \quad \text { e } \log \frac{\widehat{q}-\frac{1}{2}}{\widehat{p}+\widehat{q}-\frac{1}{2}} \doteq \log \prod_{t=1}^{n}\left(1-y_{t}\right)^{1 / n}
$$

Resolvendo as duas equações acima, encontramos valores aproximados para $\widehat{p}$ e $\widehat{q}$ dados por

$$
\begin{gathered}
\widehat{p} \doteq \frac{1}{2}\left\{\frac{1-\prod_{t=1}^{n}\left(1-y_{t}\right)^{1 / n}}{1-\prod_{t=1}^{n} y_{t}^{1 / n}-\prod_{t=1}^{n}\left(1-y_{t}\right)^{1 / n}}\right\} \mathrm{e} \\
\widehat{q} \doteq \frac{1}{2}\left\{\frac{1-\prod_{t=1}^{n} y_{t}^{1 / n}}{1-\prod_{t=1}^{n} y_{t}^{1 / n}-\prod_{t=1}^{n}\left(1-y_{t}\right)^{1 / n}}\right\} .
\end{gathered}
$$

Iniciando com esses valores, podemos encontrar soluçōes de (2.9) através de um método iterativo. Outro meio para obtenção das estimativas iniciais dos parâmetros 
é utilizar as estimativas do método dos momentos, tópico este que será abordado na próxima seção.

Resultados de convergência para os estimadores de máxima verossimilhança dos parâmetros da distribuição $\operatorname{Beta}(p, q)$ são fornecidos a seguir. Sob condições gerais de regularidade (ver, por exemplo, Sen e Singer, 1993, Capítulo 5), quando o tamanho da amostra é grande, temos que

$$
\left(\begin{array}{c}
\widehat{p} \\
\widehat{q}
\end{array}\right) \sim \mathcal{N}_{2}\left(\left(\begin{array}{c}
p \\
q
\end{array}\right), K^{-1}(p, q)\right)
$$

aproximadamente, em que $\widehat{p}$ e $\widehat{q}$ são os estimadores de máxima verossimilhança para $p$ e $q$, respectivamente. A matriz de covariâncias assintótica dos estimadores de máxima verossimilhança $K^{-1}(p, q)$ (ver Apêndice A) é dada por

$$
\begin{aligned}
K^{-1}(p, q) & =n^{-1}\left[\psi^{\prime}(p) \psi^{\prime}(q)-\psi^{\prime}(p+q)\left\{\psi^{\prime}(p)+\psi^{\prime}(q)\right\}\right]^{-1} \\
& \times\left(\begin{array}{cc}
\psi^{\prime}(q)-\psi^{\prime}(p+q) & \psi^{\prime}(p+q) \\
\psi^{\prime}(p+q) & \psi^{\prime}(p)-\psi^{\prime}(p+q)
\end{array}\right)
\end{aligned}
$$

em que $\psi^{\prime}(\lambda)$ é a função trigama, definida por

$$
\psi^{\prime}(\lambda)=\frac{\mathrm{d} \psi(\lambda)}{\mathrm{d} \lambda}=\frac{\mathrm{d}^{2} \log \Gamma(\lambda)}{\mathrm{d} \lambda^{2}}=\frac{\Gamma^{\prime \prime}(\lambda) \Gamma(\lambda)-\left[\Gamma^{\prime}(\lambda)\right]^{2}}{[\Gamma(\lambda)]^{2}}, \quad \lambda>0 .
$$

De (2.11) e (2.12), verifica-se que os estimadores de máxima verossimilhança são assintoticamente não viciados e consistentes. Também é importante notar que $(\widehat{p}, \widehat{q})$ dependem dos dados apenas através da estatística suficiente $\left(\prod_{t=1}^{n} y_{t}, \prod_{t=1}^{n}\left(1-y_{t}\right)\right)$, ou equivalentemente $\left(\sum_{t=1}^{n} \log y_{t}, \sum_{t=1}^{n} \log \left(1-y_{t}\right)\right)$.

\subsubsection{Método dos Momentos}

O método dos momentos é um dos métodos de estimação mais simples e antigo. Este método tem sido utilizado desde o século XIX, sendo introduzido por Pearson 
(1895). Seja

$$
m_{r}=\frac{1}{n} \sum_{t=1}^{n} y_{t}^{r}, \quad r \geq 1,
$$

o $r$-ésimo momento amostral de uma amostra aleatória $y_{1}, \ldots, y_{n}$ da distribuição $\operatorname{Beta}(p, q)$. Seja

$$
\mu_{r}^{\prime}=\mathrm{E}\left[y^{r}\right], \quad r \geq 1
$$

o r-ésimo momento populacional, definido em (2.5). O método dos momentos consiste na obtenção de estimadores para os parâmetros $p$ e $q$ a partir das soluçôes das equações

$$
m_{r}=\mu_{r}^{\prime} \quad r=1,2 .
$$

Das equações acima, encontram-se as seguintes soluções:

$$
\widetilde{p}=\frac{m_{1}^{2}-m_{1} m_{2}}{m_{2}-m_{1}^{2}} \quad \text { e } \tilde{q}=\frac{m_{1}-m_{2}}{m_{2}-m_{1}^{2}}-\widetilde{p}
$$

De forma alternativa, podemos reescrever os estimadores de momentos como

$$
\widetilde{p}=\frac{\bar{y}^{2}(1-\bar{y})-s^{2} \bar{y}}{s^{2}} \text { e } \widetilde{q}=\frac{\bar{y}(1-\bar{y})^{2}-s^{2}(1-\bar{y})}{s^{2}}
$$

com

$$
\bar{y}=\frac{1}{n} \sum_{t=1}^{n} y_{t} \quad \text { e } \quad s^{2}=\frac{1}{n} \sum_{t=1}^{n}\left(y_{t}-\bar{y}\right)^{2} .
$$

Os resultados de convergência para os estimadores do método dos momentos (EMM) dos parâmetros da distribuição $\operatorname{Beta}(p, q)$ são dados a seguir. Analogamente aos estimadores de máxima verossimilhança, sob condições gerais de regularidade (ver Sen e Singer, 1993, Capítulo 5), quando o tamanho da amostra é grande, temos que

$$
\left(\begin{array}{c}
\widetilde{p} \\
\widetilde{q}
\end{array}\right) \sim \mathcal{N}_{2}\left(\left(\begin{array}{c}
p \\
q
\end{array}\right), A(p, q)\right),
$$


aproximadamente, em que $\widetilde{p}$ e $\widetilde{q}$ são os estimadores de momentos para $p$ e $q$, respectivamente. A matriz de covariâncias assintótica dos estimadores do método dos momentos $A(p, q)$ (ver Apêndice B) é dada por

$$
A(p, q)=n^{-1}\left(\begin{array}{cc}
A_{p p} & A_{p q} \\
A_{q p} & A_{q q}
\end{array}\right)
$$

em que

$$
\begin{gathered}
A_{p p}=\frac{p(p+1)(p+q)\left(2 q^{3}+2 p^{2} q+4 p q^{2}+3 p^{2}+4 q^{2}+7 p q+4 p+5 q+1\right)}{q(p+q+1)(p+q+2)(p+q+3)}, \\
A_{p q}=A_{q p}=\frac{(p+1)(q+1)(p+q)\left(2 p^{2}+2 q^{2}+4 p q+p+q+1\right)}{(p+q+1)(p+q+2)(p+q+3)} \mathrm{e} \\
A_{q q}=\frac{q(q+1)(p+q)\left(2 p^{3}+2 p q^{2}+4 p^{2} q+3 q^{2}+4 p^{2}+7 p q+4 q+5 p+1\right)}{p(p+q+1)(p+q+2)(p+q+3)} .
\end{gathered}
$$

Como verificado para os estimadores de máxima verossimilhança, temos que os estimadores do método dos momentos são assintoticamente não viciados e de (2.14), assintoticamente consistentes.

\subsubsection{Eficiência Assintótica}

Nesta seção, faremos uma análise da eficiência relativa assintótica dos estimadores do método dos momentos com respeito aos estimadores de máxima verossimilhança (ver, por exemplo, Sen e Singer, 1993, Seção 5.5).

Como visto anteriormente, os estimadores de máxima verossimilhança e dos momentos têm distribuições normais assintoticamente em tomo dos verdadeiros valores dos parâmetros. Assim, para escolher entre estes estimadores necessitamos de algum

critério adicional. Considere, por exemplo, seqüencias $\left\{\widehat{\theta}_{n}^{(1)}\right\}$ e $\left\{\widehat{\theta}_{n}^{(2)}\right\}$ de estimadores 
tais que $\sqrt{n}\left(\widehat{\theta}_{n}^{(1)}-\theta\right) \stackrel{\mathcal{D}}{\rightarrow} \mathcal{N}\left(0, \sigma_{1}^{2}\right)$ e $\sqrt{n}\left(\widehat{\theta}_{n}^{(2)}-\theta\right) \stackrel{\mathcal{D}}{\rightarrow} \mathcal{N}\left(0, \sigma_{2}^{2}\right)$. Podemos utilizar as correspondentes variâncias assintóticas $\sigma_{1}^{2}$ e $\sigma_{2}^{2}$ para definir a eficiência relativa assintótica (ERA) de $\widehat{\theta}_{n}^{(2)}$ com respeito a $\widehat{\theta}_{n}^{(1)}$ como

$$
\operatorname{ERA}\left(\widehat{\theta}_{n}^{(2)} \mid \widehat{\theta}_{n}^{(1)}\right)=\frac{\sigma_{1}^{2}}{\sigma_{2}^{2}}
$$

Se $\operatorname{ERA}\left(\widehat{\theta}_{n}^{(2)} \mid \widehat{\theta}_{n}^{(1)}\right)<1$, dizemos que $\widehat{\theta}_{n}^{(1)}$ é assintoticamente mais eficiente do que $\widehat{\theta}_{n}^{(2)}$.

Em nosso caso, temos interesse em verificar a eficiência relativa assintótica dos estimadores de momentos $(\widetilde{p}, \widetilde{q})$ em relação aos estimadores de máxima verossimilhança $(\widehat{p}, \widehat{q})$, ou seja, verificar se

$$
\operatorname{ERA}(\widehat{p} \mid \widehat{p})=\frac{\operatorname{Var}(\widehat{p})}{\operatorname{Var}(\widehat{p})} \quad \text { e } \operatorname{ERA}(\widehat{q} \mid \widehat{q})=\frac{\operatorname{Var}(\widehat{q})}{\operatorname{Var}(\widetilde{q})}
$$

são menores ou maiores do que 1. Os valores para $\operatorname{Var}(\widehat{p})$ e $\operatorname{Var}(\widehat{q})$ são fornecidos de (2.12), enquanto que os valores de $\operatorname{Var}(\widetilde{p})$ e $\operatorname{Var}(\widetilde{q})$ vêm de (2.14). Como o cálculo analítico para as duas quantidades é muito trabalhoso, decidimos analisar o comportamento das duas quantidades para diferentes valores de $p$ e $q$.

A Tabela 2.1 apresenta o resultado obtido no estudo de eficiência relativa assintótica do estimador do método dos momentos em relação ao estimador de máxima verossimilhança para o parâmetro $p$. O resultado para o parâmetro $q$ é o mesmo obtido para o parâmetro $p$, mas com os parâmetros $p$ e $q$ permutados, ou seja, com o parâmetro $p$ nas colunas e o parâmetro $q$ nas linhas. Um fato importante observado na Tabela 2.1 é que os valores obtidos são todos menores do que ou iguais a 1 , indicando que os estimadores de momentos $(\widetilde{p}, \widetilde{q})$ são, em geral, assintoticamente menos eficientes que os estimadores de máxima verossimilhança $(\widehat{p}, \widehat{q})$. Os valores destacados na Tabela 2.1 indicam para quais valores de $p$ e $q$ os estimadores dos momentos são comparáveis aos estimadores de máxima verossimilhança em relação à eficiència assintótica (ERA > 0,990). 
Tabela 2.1

Eficiência relativa assintótica do estimador do método dos momentos

$(\widehat{p})$ em relação ao estimador de máxima verossimilhança $(\widehat{p})$.

\begin{tabular}{cccccccc}
\hline \hline & \multicolumn{7}{c}{$q$} \\
\cline { 2 - 8 }$p$ & 0,01 & 0,50 & 1 & 10 & 50 & 100 & 1000 \\
\hline \hline 0,01 & 0,042 & 0,027 & 0,020 & 0,007 & 0,005 & 0,005 & 0,005 \\
0,50 & 0,562 & 0,648 & 0,609 & 0,312 & 0,245 & 0,236 & 0,228 \\
1 & 0,629 & 0,774 & 0,803 & 0,523 & 0,418 & 0,403 & 0,389 \\
10 & 0,416 & 0,539 & 0,656 & 0,994 & 0,934 & 0,911 & 0,883 \\
50 & 0,352 & 0,446 & 0,544 & 0,938 & 1,000 & 0,995 & 0,978 \\
100 & 0,343 & 0,434 & 0,527 & 0,915 & 0,996 & 1,000 & $\mathbf{0 , 9 9 0}$ \\
1000 & 0,335 & 0,422 & 0,512 & 0,888 & 0,978 & 0,990 & $\mathbf{1 , 0 0 0}$ \\
\hline \hline
\end{tabular}

Adicionalmente à definição de eficiência relativa assintótica de um estimador em relação a outro, podemos definir a eficiência assintótica de um estimador $\widehat{\theta}$, não viciado para o parâmetro $\theta$, como

$$
e(\widehat{\theta})=\frac{L I(\theta)}{\operatorname{Var}(\widehat{\theta})}
$$

em que $L I(\theta)$ é o limite inferior de Cramér-Rao para a variância dos estimadores não viciados de $\theta$. Convém notar que se $e(\widehat{\theta})=1$, ou seja, quando a variância de $\widehat{\theta}$ coincide com o limite inferior da variância dos estimadores não viciados de $\theta, \widehat{\theta}$ é dito ser eficiente.

Sob condições gerais de regularidade (Sen e Singer, 1993), os estimadores de máxima verossimilhança são eficientes assintoticamente, ou seja, verifica-se que a variância assintótica dos estimadores de máxima verossimilhança coincide com o limite inferior de Cramér-Rao para a variância dos estimadores não viciados. Assim, a. eficiência assintótica do estimador do método dos momentos $\widetilde{p}$ para diversos valores de $p$ e $q$ são os mesmos dados na Tabela 2.1. 


\subsection{Exemplos}

Nesta seção apresentamos dois exemplos nos quais a variável de interesse é limitada ao intervalo unitário. Os dois exemplos ilustram conjuntos de dados reais que serão analisados no Capítulo 5 através do modelo de regressão beta.

\subsubsection{Oxidação de Amônia}

Os dados da Tabela 2.2 foram obtidos em 21 dias de processos de oxidação de amônia como um estágio para a produção do ácido nítrico em uma planta industrial. O ácido nítrico é utilizado na produção de fertilizantes, corantes, medicamentos, explosivos, etc.

Tabela 2.2

Dados do estudo de Oxidação de Amônia.

\begin{tabular}{ccccc}
\hline \hline Observação & $\begin{array}{c}\text { Perda na } \\
\text { conversāo } \\
y\end{array}$ & $\begin{array}{c}\text { Corrente } \\
\text { de ar } \\
x_{1}\end{array}$ & $\begin{array}{c}\text { Temperatura } \\
\text { da água } \\
x_{2}\end{array}$ & $\begin{array}{c}\text { Concentração } \\
\text { de ácido } \\
x_{3}\end{array}$ \\
\hline 1 & 0,042 & 80 & 27 & 89 \\
2 & 0,037 & 80 & 27 & 88 \\
3 & 0,037 & 75 & 25 & 90 \\
4 & 0,028 & 62 & 24 & 87 \\
5 & 0,018 & 62 & 22 & 87 \\
6 & 0,018 & 62 & 23 & 87 \\
7 & 0,019 & 62 & 24 & 93 \\
8 & 0,020 & 62 & 24 & 93 \\
9 & 0,015 & 58 & 23 & 87 \\
10 & 0,014 & 58 & 18 & 80 \\
11 & 0,014 & 58 & 18 & 89 \\
12 & 0,013 & 58 & 17 & 88 \\
13 & 0,011 & 58 & 18 & 82 \\
14 & 0,012 & 58 & 19 & 93 \\
15 & 0,008 & 50 & 18 & 89 \\
16 & 0,007 & 50 & 18 & 86 \\
17 & 0,008 & 50 & 19 & 72 \\
18 & 0,008 & 50 & 19 & 79 \\
19 & 0,009 & 50 & 20 & 80 \\
20 & 0,015 & 56 & 20 & 82 \\
21 & 0,015 & 70 & 20 & 91 \\
\hline \hline
\end{tabular}


Brownlee (1965, p. 454) apresenta este conjunto de dados como um exemplo de aplicação de regressão linear múltipla.

A variável dependente, perda na conversão de amônia, é a proporção de amônia que não foi convertida em ácido nítrico, isto é, uma medida inversa da eficiência total da planta industrial. As variáveis explicativas são: a corrente de ar, a temperatura da água utilizada para o resfriamento da reação e a concentração de ácido, esta dada por $10 \times($ concentração de ácido - 50). Por exemplo, 89 corresponde a $58,9 \%$ de concentração de ácido. Esses dados foram extensivamente analisados na literatura estatística. Algumas referências são dadas no Capítulo 5.

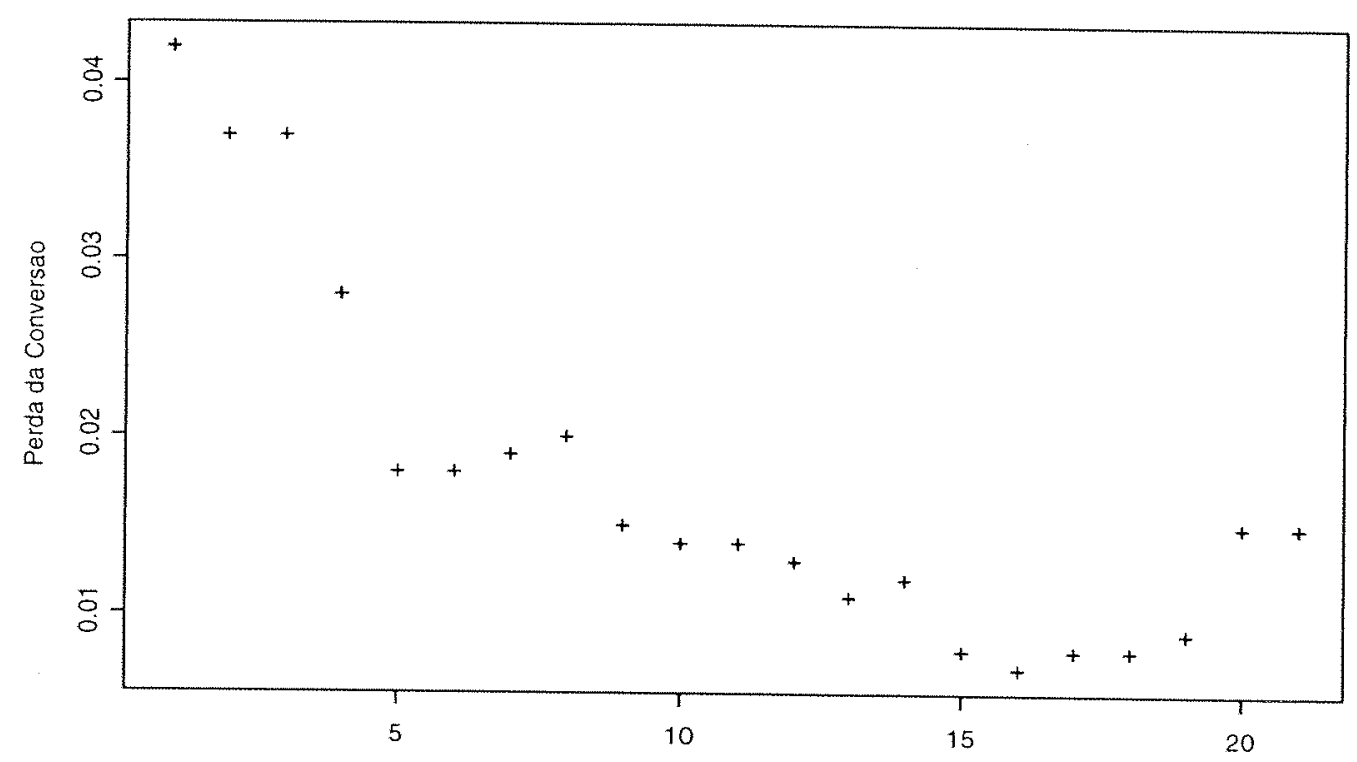

Figura 2.2: Gráfico das perdas de conversão versus o índice das observaçôes.

Na Figura 2.2 apresentamos o gráfico das perdas de conversão versus o índice das observaçōes. Atkinson $(1985$, p. 130) comenta que, como a variável correspondente à perda na conversão é necessariamente não negativa, é possível que uma transformação 
da variável resposta seja conveniente para uma análise de regressão linear. A nossa proposta é analisar este conjunto de dados assumindo uma distribuição beta para a variável resposta original, sem transformação, ajustando então, o modelo de regressão beta proposto no próximo capítulo. No Capítulo 5 discutiremos com mais detalhes esse conjunto de dados.

\subsubsection{Contaminação de Amendoim}

Draper e Smith (1998, p. 101-102) apresentam um conjunto de dados que consiste de 34 lotes de 120 libras (aproximadamente $54 \mathrm{~kg}$ ) de amendoins. Os dados estão apresentados na Tabela 2.3 .

Tabela 2.3

Dados do estudo da Contaminação de Amendoim.

\begin{tabular}{cccccc}
\hline Obs. & $\begin{array}{c}\text { Proporção de amendoins } \\
\text { não contaminados }\end{array}$ & $\begin{array}{c}\text { Nível de } \\
\text { aflatoxina } \\
x\end{array}$ & $\begin{array}{c}\text { Obs. } \\
y\end{array}$ & $\begin{array}{c}\text { Proporção de amendoins } \\
\text { nāo contaminados }\end{array}$ & $\begin{array}{c}\text { Nível de } \\
\text { aflatoxina } \\
x\end{array}$ \\
\hline \hline 1 & 0,99971 & 3,0 & 18 & 0,99933 & 24,2 \\
2 & 0,99979 & 4,7 & 19 & 0,99858 & 25,8 \\
3 & 0,99982 & 8,3 & 20 & 0,99987 & 30,6 \\
4 & 0,99971 & 9,3 & 21 & 0,99958 & 36,2 \\
5 & 0,99957 & 9,9 & 22 & 0,99909 & 39,8 \\
6 & 0,99961 & 11,0 & 23 & 0,99859 & 44,3 \\
7 & 0,99956 & 12,3 & 24 & 0,99863 & 46,8 \\
8 & 0,99972 & 12,5 & 25 & 0,99811 & 46,8 \\
9 & 0,99889 & 12,6 & 26 & 0,99877 & 58,1 \\
10 & 0,99961 & 15,9 & 27 & 0,99798 & 62,3 \\
11 & 0,99982 & 16,7 & 28 & 0,99855 & 70,6 \\
12 & 0,99975 & 18,8 & 29 & 0,99788 & 71,1 \\
13 & 0,99942 & 18,8 & 30 & 0,99821 & 71,3 \\
14 & 0,99932 & 18,9 & 31 & 0,99830 & 83,2 \\
15 & 0,99908 & 21,7 & 32 & 0,99718 & 83,6 \\
16 & 0,99970 & 21,9 & 33 & 0,99642 & 99,5 \\
17 & 0,99985 & 22,8 & 34 & 0,99658 & 111,2 \\
\hline \hline
\end{tabular}

Para cada lote é fornecido o nível (médio) de aflatoxina ${ }^{1}(x)$, em partes por bilhào

\footnotetext{
${ }^{1}$ Aflatoxina é a denominação dada a um grupo de substancias, muito semelhantes, e que são tóxicas para o homem e para os animais.
} 
(ppb), e é medida a proporção de amendoins não contaminados no lote $(y)$. O objetivo é investigar a relação ente as duas variáveis e predizer $y$ através de $x$.

Na Figura 2.3 ilustramos o gráfico da variável resposta "proporção de amendoins não contaminados" contra a variável explicativa "nível de aflatoxina". Podemos observar que todos os valores da variável resposta se encontram muito próximos de 1 . Nota-se que, com o aumento do nível de aflatoxina, há uma tendência de decréscimo na proporção de amendoins não contaminados, como esperado.

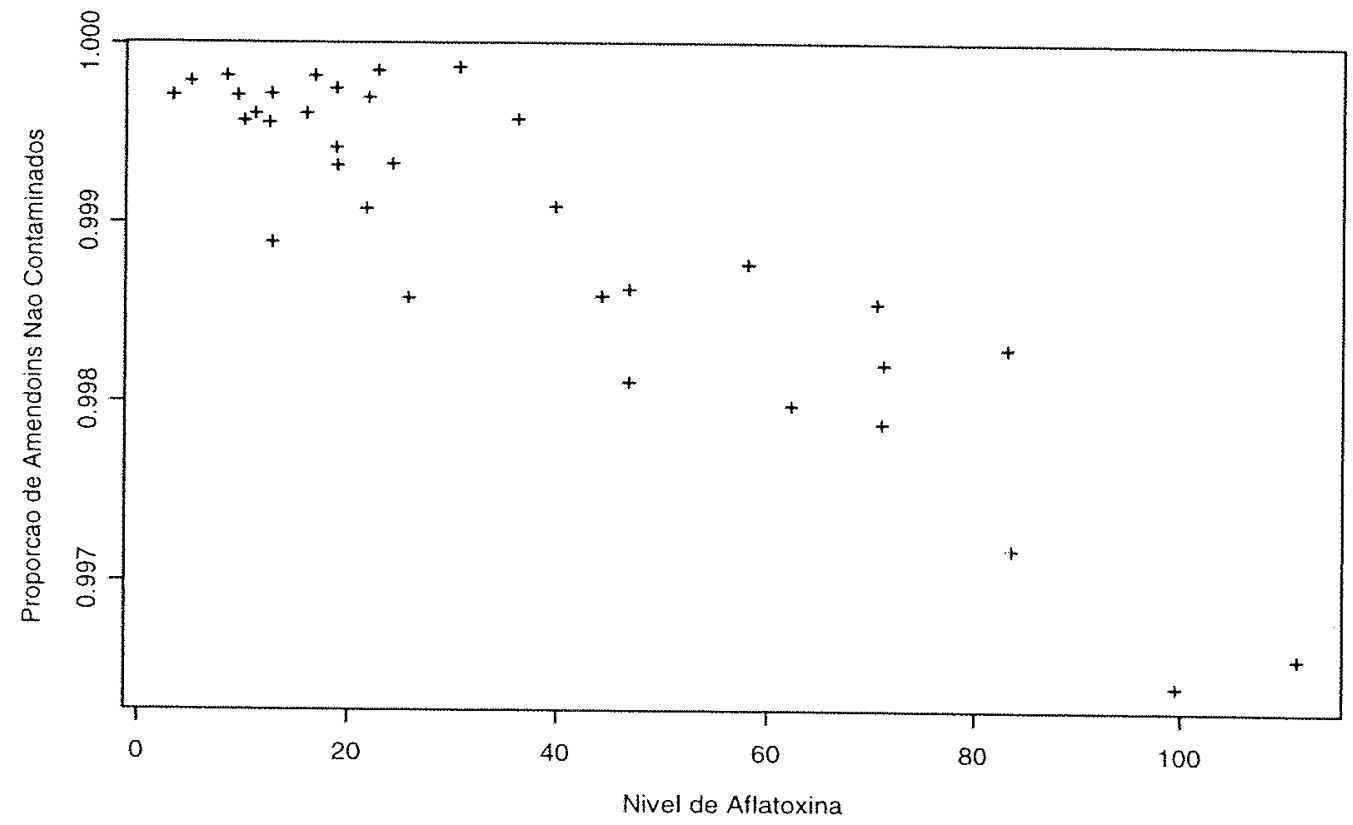

Figura 2.3: Gráfico das proporções de amendoins năo contaminados contra o nivel de aflatoxina.

Draper e Smith (1981) propöem analisar este conjunto de dados através de uma regressão linear simples. No Capítulo 5, analisaremos este conjunto através da regressão linear e da regressão beta a fim de comparar os ajustes. Realizaremos uma análise adicional, simulando a variável resposta y de uma distribuição beta a 
partir da variável explicativa $x$, para ilustrar que a regressão linear pode produzir valores ajustados fora do intervalo $(0,1)$. Nessa situação, apresentaremos o ajuste da regressão beta e compararemos novamente os resultados. 
Capítulo 3

\section{Modelo de Regressão Beta}

\subsection{Introdução}

Neste capítulo apresentamos o modelo de regressão beta proposto por Ferrari e Cribari-Neto (2004). Este modelo é utilizado para situações em que a variável de interesse é contínua e restrita ao intervalo $(0,1)$ e está relacionada a outras variáveis numa estrutura de regressão, como é o caso dos exemplos discutidos na Seção 2.4.

Este capítulo foi escrito baseado primordialmente no artigo de Ferrari e CribariNeto (2004). Em seu desenvolvimento, apresentamos além do modelo de regressão beta, algumas funções de ligação, a função escore e matriz de informação, a estimação dos parâmetros de regressão, testes de hipóteses, intervalos de confiança e algumas medidas de diagnóstico. 


\subsection{Definição}

A densidade da distribuição beta definida em (2.1) é indexada por dois parâmetros: $p$ e $q$. No entanto, para análise de regressão é usual modelar a média da resposta em termos de outros parâmetros e variáveis explicativas e também definir um modelo que contenha um parâmetro de precisão (ou dispersão). Com o objetivo de obter uma estrutura de regressão para a média da resposta juntamente com um parâmetro de precisão, uma parametrização diferente da densidade beta foi sugerida por Ferrari e Cribari-Neto (2004). Seja $\mu=p /(p+q)$ e $\phi=p+q$, isto é, $p=\mu \phi$ e $q=(1-\mu) \phi$. Segue de (2.6) e (2.7) que

$$
\mathrm{E}(y)=\mu \quad \text { e } \operatorname{Var}(y)=\frac{V(\mu)}{1+\phi},
$$

em que $V(\mu)=\mu(1-\mu)$, de tal forma que $\mu$ é a média da variável $y$ e $\phi$ pode ser interpretado como um parâmetro de precisāo no sentido que, para $\mu$ fixado, quanto maior o valor de $\phi$, menor a variância de $y$. A densidade de $y$ pode ser escrita, na nova parametrização como

$$
f(y ; \mu, \phi)=\frac{\Gamma(\phi)}{\Gamma(\mu \phi) \Gamma((1-\mu) \phi)} y^{\mu \phi-1}(1-y)^{(1-\mu) \phi-1}, \quad 0<y<1,
$$

em que $0<\mu<1$ e $\phi>0$.

A Figura 3.1 apresenta algumas densidades beta, juntamente com os correspondentes valores de $(\mu, \phi)$. Nota-se que a densidade beta pode apresentar diferentes formas dependendo dos valores dos parâmetros $(\mu, \phi)$. Em particular, pode ser simétrica, quando $\mu=1 / 2$, ou assimétrica, quando $\mu \neq 1 / 2$. Adicionalmente, a dispersão da distribuição, para $\mu$ fixado, decresce quando $\phi$ cresce (ver Figuras 3.1 (a), (b) e (c)). Outro fato interessante é que a Figura 3.1 (a) apresenta uma densidade com o formato $J$ e outra densidade com o formato $J$ invertido. Este mesmo fato ocorre na Figura 3.1 (b). Na Figura 3.1 (d) é apresentado o caso uniforme $(\mu=0.5 ; \phi=2)$ e as densidades com formato $U$, simétricas e assimétricas. 

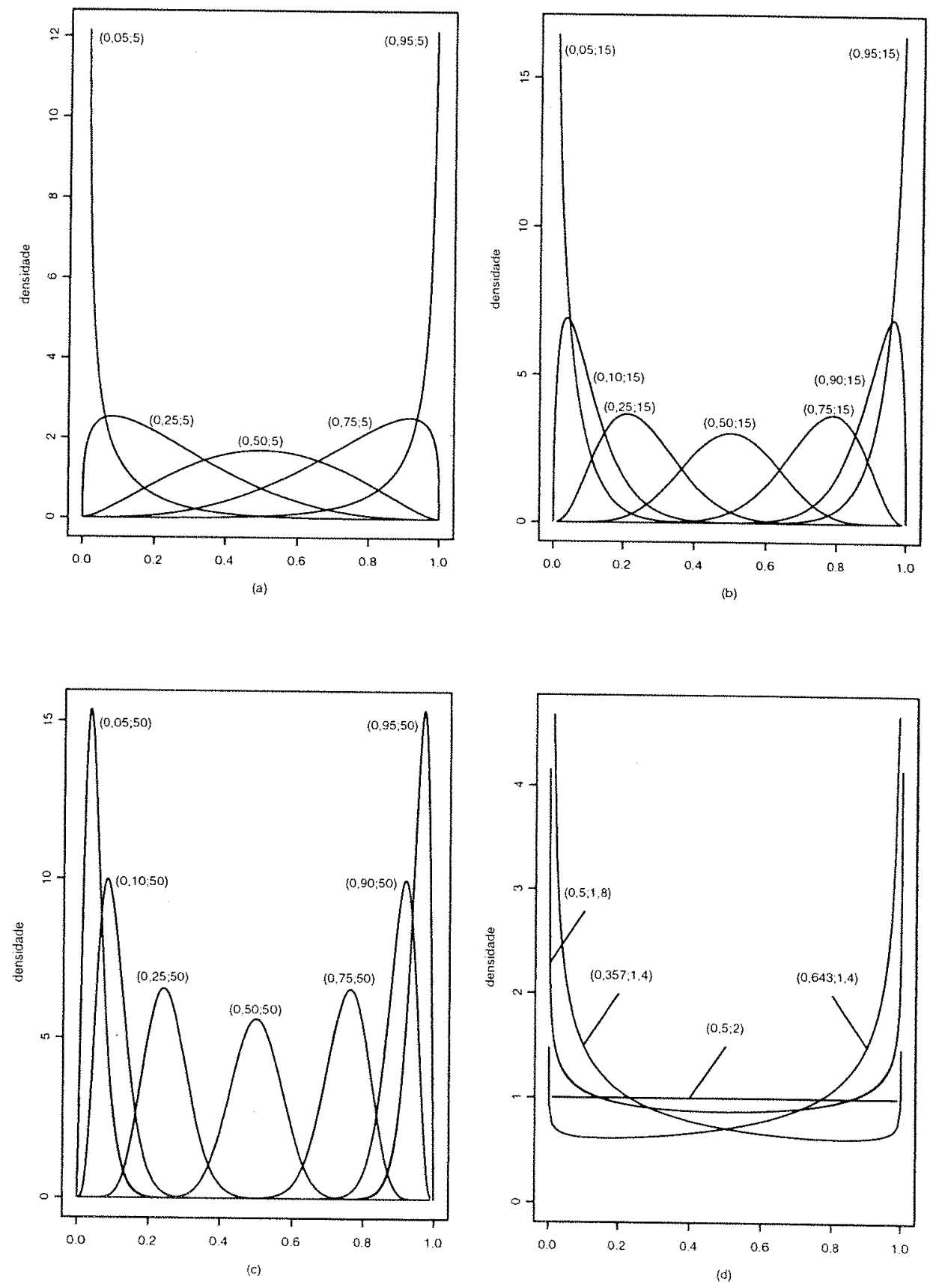

Figura 3.1: Densidades beta para diferentes combinações de $(\mu, \phi)$ 
Assumimos que a variável resposta está restrita ao intervalo unitário padrão $(0,1)$. No entanto, o modelo proposto é utilizado em situações mais gerais, em que a resposta é restrita ao intervalo $(a, b)$ e $a$ e $b$ são constantes conhecidas, $a<b$. Neste caso, modela-se $(y-a) /(b-a)$ em vez de modelar $y$ diretamente.

Sejam $y_{1}, \ldots, y_{n}$ variáveis aleatórias independentes, em que cada $y_{t}, t=1, \ldots, n$, tem a densidade como em (3.1) com média $\mu_{t}$ e parâmetro de precisão desconhecido $\phi$. O modelo de regressão beta é definido por (3.1) e pelo componente sistemático

$$
g\left(\mu_{t}\right)=\sum_{i=1}^{k} x_{i i} \beta_{i}=\eta_{t}
$$

em que $\eta_{t}=x_{t}^{\top} \beta$ é o preditor linear, $\beta=\left(\beta_{1}, \ldots, \beta_{k}\right)^{\top}$ é um vetor de parâmetros desconhecidos a serem estimados $\left(\beta \in \mathbb{R}^{k}\right), x_{t}^{\top}=\left(x_{t 1}, \ldots, x_{t k}\right)$ representa os valores de $k(k<n)$ variáveis explicativas que são assumidas fixas e conhecidas e $g(\cdot)$ é uma função estritamente monótona e duplamente diferenciável que transforma valores do intervalo $(0,1)$ em $\mathbb{R}$, denominada função de ligação. Note que a variância de $y_{t}$ é uma função de $\mu_{t}$ e, como uma conseqüência de (3.2), dos valores das variáveis explicativas. Logo, variáveis respostas $\left(y_{t}\right)$ de variâncias não constantes são naturalmente acomodadas no modelo.

\subsection{Funções de Ligação}

Existem algumas possíveis escolhas para a função de ligação $g(\cdot)$. Por exemplo, podemos utilizar a especificação $\operatorname{logito} g(\mu)=\log \{\mu /(1-\mu)\}$, a função probito $g(\mu)=\Phi^{-1}(\mu)$, em que $\Phi(\cdot)$ é a função de distribuição acumulada de uma variável aleatória normal padrão, a ligação complemento $\log -\log g(\mu)=\log \{-\log (1-\mu)\}$, a ligação $\log -\log g(\mu)=-\log \{-\log (\mu)\}$, entre outras. Todas essas quatro funçōes de ligações são contínuas e estritamente crescentes em $(0,1)$. Uma comparação dessas 
funçōes de ligação foi feita por McCullagh e Nelder (1989, Seção 4.3.1). Mais detalhes sobre outras transformações, ver Atkinson (1985, Cap. 7).

Utilizando a função de ligação logito em (3.2), podemos escrever

$$
g\left(\mu_{t}\right)=\log \left(\frac{\mu_{t}}{1-\mu_{t}}\right)=x_{i}^{\top} \beta, \quad t=1, \ldots, n .
$$

Equivalentemente, o modelo pode ser escrito como

$$
\frac{\mu_{t}}{1-\mu_{t}}=\exp \left(x_{t}^{\top} \beta\right)
$$

ou ainda como

$$
\mu_{t}=\frac{\exp \left(x_{t}^{\top} \beta\right)}{1+\exp \left(x_{t}^{\top} \beta\right)}
$$

A equação (3.3) é a função inversa de $g\left(\mu_{t}\right)$. Aqui, os parâmetros de regressão têm uma importante interpretação. Suponha que o valor da $i$-ésima variável regressora é aumentado por $c$ unidades e todas as outras variáveis independentes permanecem inalteradas. Seja $\mu^{\dagger}$ a média de $y$ sob este novo valor das covariadas, uma vez que $\mu$ denota a média de $y$ sob o valor original das covariadas. Temos então que,

$$
\frac{\mu^{\dagger}}{1-\mu^{\dagger}}=\exp \left(x_{t 1} \beta_{1}+\cdots+\left(x_{t i}+c\right) \beta_{i}+\cdots+x_{t k} \beta_{k}\right)
$$

e agora é fácil verificar que

$$
\exp \left(c \beta_{i}\right)=\frac{\mu^{\dagger} /\left(1-\mu^{\dagger}\right)}{\mu /(1-\mu)}
$$

isto é, $\exp \left\{c \beta_{i}\right\}$ é a razão de chances (odds ratio). Considere o exemplo de oxidação de amônia, introduzido na Seção 2.4.1, e defina a chance de não conversão de amônia, como sendo o quociente entre a proporção média de amônia não convertida dividida pela proporção média de amônia convertida. Como uma ilustraçāo, se, em média, $10 \%$ da amônia não é convertida em ácido nítrico, então a chance de não conversão é $1 / 9$. Um quociente menor do que 1 significa que ocorre mais conversão do que 
não conversão, mas se o valor for maior do que 1 , significa que ocorre menos conversão do que não conversão, sendo este fato desfavorável para a planta industrial. Suponha que a temperatura da água, utilizada para o resfriamento da reação em que a amônia é convertida em ácido nítrico, aumente em $2^{\circ} \mathrm{C}$, então duas vezes o parâmetro de regressão associado para esta covariável pode ser interpretado como o logaritmo da razão de chances de não conversão da amônia, com todas as outras variáveis permanecendo constantes.

\subsection{Função Escore e Matriz de Informação}

Esta seção apresenta a função escore e a matriz de informação de Fisher para o vetor de parâmetros $\beta$ e também para o parâmetro de precisão $\phi$.

O logaritmo natural da função de verossimilhança baseada em uma amostra de $n$. observaçōes independentes é

$$
\ell(\beta, \phi)=\sum_{t=1}^{n} \ell_{t}\left(\mu_{t}, \phi\right)
$$

em que

$$
\begin{aligned}
\ell_{t}\left(\mu_{t}, \phi\right) & =\log \Gamma(\phi)-\log \Gamma\left(\mu_{t} \phi\right)-\log \Gamma\left(\left(1-\mu_{t}\right) \phi\right)+\left(\mu_{t} \phi-1\right) \log y_{t} \\
& +\left\{\left(1-\mu_{t}\right) \phi-1\right\} \log \left(1-y_{t}\right)
\end{aligned}
$$

$\operatorname{com} \mu_{t}=g^{-1}\left(\eta_{t}\right)$ definido em (3.2). A função escore é obtida derivando o logaritmo da função de verossimilhança com respeito aos parâmetros desconhecidos. Segue que, para $i=1, \ldots, k$,

$$
\frac{\partial \ell(\beta, \phi)}{\partial \beta_{i}}=\sum_{t=1}^{n} \frac{\partial \ell_{t}\left(\mu_{t}, \phi\right)}{\partial \mu_{t}} \frac{\mathrm{d} \mu_{t}}{\mathrm{~d} \eta_{t}} \frac{\partial \eta_{t}}{\partial \beta_{i}}
$$

com

$$
\frac{\partial \ell_{t}\left(\mu_{t}, \phi\right)}{\partial \mu_{t}}=\phi\left[\log \frac{y_{t}}{1-y_{t}}-\left\{\psi\left(\mu_{t} \phi\right)-\psi\left(\left(1-\mu_{t}\right) \phi\right)\right\}\right]
$$


em que $\psi(\cdot)$ é a função digama, definida em (2.10). Note que $\mathrm{d} \mu_{t} / \mathrm{d} \eta_{t}=1 / g^{\prime}\left(\mu_{t}\right)$ e também $\partial \eta_{t} / \partial \beta_{i}=x_{t i}$.

Antes de obter a expressão final para (3.5), sejam $y_{t}^{*}=\log \left\{y_{t} /\left(1-y_{t}\right)\right\}$ e $\mu_{t}^{*}=$ $\psi\left(\mu_{t} \phi\right)-\psi\left(\left(1-\mu_{t}\right) \phi\right)$. Assim, a expressão (3.6) pode ser reescrita como

$$
\frac{\partial \ell_{t}\left(\mu_{t}, \phi\right)}{\partial \mu_{t}}=\phi\left(y_{t}^{*}-\mu_{t}^{*}\right)
$$

e, finalmente, (3.5) reduz-se a

$$
\frac{\partial \ell(\beta, \phi)}{\partial \beta_{i}}=\phi \sum_{t=1}^{n}\left(y_{t}^{*}-\mu_{t}^{*}\right) \frac{1}{g^{\prime}\left(\mu_{t}\right)} x_{t i} .
$$

Note também que, assumindo condiçōes usuais de regularidade (ver, Sen e Singer, 1993, Capítulo 7$)$, o valor esperado de $\partial \ell_{t}\left(\mu_{t}, \phi\right) / \partial \mu_{t}$ é igual a zero. Mas,

$$
\mathrm{E}\left[\frac{\partial \ell_{t}\left(\mu_{t}, \phi\right)}{\partial \mu_{t}}\right]=0 \Leftrightarrow \mathrm{E}\left[\phi\left(y_{t}^{*}-\mu_{t}^{*}\right)\right]=0 \Leftrightarrow \mathrm{E}\left[y_{t}^{*}\right]=\mu_{t}^{*},
$$

ou seja, o valor esperado da variável transformada $y_{t}^{*}$ iguala-se a $\mu_{t}^{*}$.

A função escore para $\beta$, o vetor coluna $U_{\beta}(\beta, \phi)$ de dimensão $k$, pode ser escrita na seguinte forma matricial:

$$
U_{\beta}(\beta, \phi)=\phi X^{\top} T\left(y^{*}-\mu^{*}\right)
$$

em que $X$ é uma matriz $(n \times k)$ cuja t-ésima linha é $x_{t}^{\top}, T=\operatorname{diag}\left\{1 / g^{\prime}\left(\mu_{1}\right), \ldots\right.$, $\left.1 / g^{\prime}\left(\mu_{n}\right)\right\}, y^{*}=\left(y_{1}^{*}, \ldots, y_{n}^{*}\right)^{\top}$ e $\mu^{*}=\left(\mu_{1}^{*}, \ldots, \mu_{n}^{*}\right)^{\top}$.

Analogamente, a derivada de $1^{a}$ ordem do logaritmo da função de verossimilhança em relação a $\phi$ é dada por

$$
\frac{\partial \ell(\beta, \phi)}{\partial \phi}=\sum_{t=1}^{n} \frac{\partial \ell_{t}\left(\mu_{t}, \phi\right)}{\partial \phi}
$$

com

$$
\begin{aligned}
\frac{\partial \ell_{t}\left(\mu_{t,} \phi\right)}{\partial \phi}= & \mu_{t}\left[\log \frac{y_{t}}{1-y_{t}}-\psi\left(\mu_{t} \phi\right)+\psi\left(\left(1-\mu_{t}\right) \phi\right)\right] \\
& +\log \left(1-y_{t}\right)-\psi\left(\left(1-\mu_{t}\right) \phi\right)+\psi(\phi) .
\end{aligned}
$$


Portanto, a função escore para o parâmetro de precisão $\phi$ é dada por

$$
U_{\phi}(\beta, \phi)=\sum_{t=1}^{n}\left\{\mu_{t}\left(y_{t}^{*}-\mu_{t}^{*}\right)+\log \left(1-y_{t}\right)-\psi\left(\left(1-\mu_{t}\right) \phi\right)+\psi(\phi)\right\},
$$

sendo $U_{\phi}(\beta, \phi)$ um escalar.

O nosso próximo passo é obter a matriz de informação de Fisher para $(\beta, \phi)$. De (3.5), as derivadas de $2^{a}$ ordem de $\ell(\beta, \phi)$ com respeito a $\beta_{i}$ e $\beta_{j}$ são dadas por

$$
\begin{aligned}
\frac{\partial^{2} \ell(\beta, \phi)}{\partial \beta_{i} \partial \beta_{j}} & =\sum_{t=1}^{n} \frac{\partial}{\partial \mu_{t}}\left(\frac{\partial \ell_{t}\left(\mu_{t}, \phi\right)}{\partial \mu_{t}} \frac{\mathrm{d} \mu_{t}}{\mathrm{~d} \eta_{t}}\right) \frac{\mathrm{d} \mu_{t}}{\mathrm{~d} \eta_{t}} \frac{\partial \eta_{t}}{\partial \beta_{j}} x_{t i} \\
& =\sum_{t=1}^{n}\left\{\frac{\partial^{2} \ell_{t}\left(\mu_{t}, \phi\right)}{\partial \mu_{t}^{2}} \frac{\mathrm{d} \mu_{t}}{\mathrm{~d} \eta_{t}}+\frac{\partial \ell_{t}\left(\mu_{t}, \phi\right)}{\partial \mu_{t}} \frac{\partial}{\partial \mu_{t}}\left(\frac{\mathrm{d} \mu_{t}}{\mathrm{~d} \eta_{t}}\right)\right\} \frac{\mathrm{d} \mu_{t}}{\mathrm{~d} \eta_{t}} x_{t i} x_{t j}
\end{aligned}
$$

Como $\mathrm{E}\left(\partial \ell_{t}\left(\mu_{t}, \phi\right) / \partial \mu_{t}\right)=0$, temos

$$
\mathrm{E}\left(\frac{\partial^{2} \ell(\beta, \phi)}{\partial \beta_{i} \partial \beta_{j}}\right)=\sum_{t=1}^{n} \mathrm{E}\left(\frac{\partial^{2} \ell_{t}\left(\mu_{t}, \phi\right)}{\partial \mu_{t}^{2}}\right)\left(\frac{\mathrm{d} \mu_{t}}{\mathrm{~d} \eta_{t}}\right)^{2} x_{t i} x_{t j}
$$

Agora, de (3.6) temos

$$
\frac{\partial^{2} \ell_{t}\left(\mu_{t}, \phi\right)}{\partial \mu_{t}^{2}}=-\phi^{2}\left\{\psi^{\prime}\left(\mu_{t} \phi\right)+\psi^{\prime}\left(\left(1-\mu_{t}\right) \phi\right)\right\}
$$

em que $\psi^{\prime}(\cdot)$ é a função trigama definida em (2.13) e assim

$$
\mathrm{E}\left(\frac{\partial^{2} \ell(\beta, \phi)}{\partial \beta_{i} \partial \beta_{j}}\right)=-\phi \sum_{t=1}^{n} w_{t} x_{t i} x_{t j}
$$

$\mathrm{com}$

$$
w_{t}=\phi\left\{\psi^{\prime}\left(\mu_{t} \phi\right)+\psi^{\prime}\left(\left(1-\mu_{t}\right) \phi\right)\right\} \frac{1}{\left\{g^{\prime}\left(\mu_{t}\right)\right\}^{2}} .
$$

Em forma matricial, temos

$$
\mathrm{E}\left(\frac{\partial^{2} \ell(\beta, \phi)}{\partial \beta \partial \beta^{\top}}\right)=-\phi X^{\top} W X
$$

$\operatorname{com} W=\operatorname{diag}\left\{w_{1}, \ldots, w_{n}\right\}$ 
De (3.7), a derivada de $2^{\underline{a}}$ ordem de $\ell(\beta, \phi)$ com respeito a $\beta_{i}$ e $\phi$ pode ser escrita como

$$
\frac{\partial^{2} \ell(\beta, \phi)}{\partial \beta_{i} \partial \phi}=\sum_{t=1}^{n}\left[\left(y_{t}^{*}-\mu_{t}^{*}\right)-\phi \frac{\partial \mu_{t}^{*}}{\partial \phi}\right] \frac{1}{g^{\prime}\left(\mu_{t}\right)} x_{t i}
$$

Como $\mathrm{E}\left(y_{t}^{*}\right)=\mu_{t}^{*}$ e $\partial \mu_{t}^{*} / \partial \phi=\psi^{\prime}\left(\mu_{t} \phi\right) \mu_{t}-\psi^{\prime}\left(\left(1-\mu_{t}\right) \phi\right)\left(1-\mu_{t}\right)$, chegamos a

$$
\mathrm{E}\left(\frac{\partial^{2} \ell(\beta, \phi)}{\partial \beta_{i} \partial \phi}\right)=-\sum_{t=1}^{n} c_{t} \frac{1}{g^{\prime}\left(\mu_{t}\right)} x_{t i}
$$

em que $c_{t}=\phi\left\{\psi^{\prime}\left(\mu_{t} \phi\right) \mu_{t}-\psi^{\prime}\left(\left(1-\mu_{t}\right) \phi\right)\left(1-\mu_{t}\right)\right\}$. Em notação matricial,

$$
\mathrm{E}\left(\frac{\partial^{2} \ell(\beta, \phi)}{\partial \beta \partial \phi}\right)=-X^{\top} T c
$$

$\operatorname{com} c=\left(c_{1}, \ldots, c_{n}\right)^{\top}$

Finalmente, temos que $\partial^{2} \ell(\beta, \phi) / \partial \phi^{2}$ pode ser obtido diferenciando a expressão em (3.9) com respeito a $\phi$, ou seja,

$$
\begin{aligned}
\frac{\partial^{2} \ell(\beta, \phi)}{\partial \phi^{2}} & =\sum_{t=1}^{n} \frac{\partial^{2} \ell_{t}\left(\mu_{t}, \phi\right)}{\partial \phi^{2}} \\
& =-\sum_{t=1}^{n}\left[\psi^{\prime}\left(\mu_{t} \phi\right) \mu_{t}^{2}+\psi^{\prime}\left(\left(1-\mu_{t}\right) \phi\right)\left(1-\mu_{t}\right)^{2}-\psi^{\prime}(\phi)\right] .
\end{aligned}
$$

Seja $d_{t}=\psi^{\prime}\left(\mu_{t} \phi\right) \mu_{t}^{2}+\psi^{\prime}\left(\left(1-\mu_{t}\right) \phi\right)\left(1-\mu_{t}\right)^{2}-\psi^{\prime}(\phi)$. Logo,

$$
\mathrm{E}\left(\frac{\partial^{2} \ell(\beta, \phi)}{\partial \phi^{2}}\right)=-\sum_{t=1}^{n} d_{t}
$$

que, em notação matricial, pode ser escrito como

$$
E\left(\frac{\partial^{2} \ell(\beta, \phi)}{\partial \phi^{2}}\right)=-\operatorname{tr}(D)
$$

em que $D=\operatorname{diag}\left(d_{1}, \ldots, d_{n}\right) \operatorname{etr}(D)$ indica o traço da matriz $D$.

Finalmente, a matriz de informação de Fisher para $(\beta, \phi)$ é dada por

$$
K=K(\beta, \phi)=\left(\begin{array}{cc}
K_{\beta \beta} & K_{\beta \phi} \\
K_{\phi \beta} & K_{\phi \phi}
\end{array}\right)
$$


em que $K_{\beta \beta}=\phi X^{\top} W X, K_{\beta \phi}=K_{\phi \beta}^{\top}=X^{\top} T c$, e $K_{\phi \phi}=\operatorname{tr}(D)$. Note que os parâmetros $\beta$ e $\phi$ não são ortogonais, em contraste ao que é verificado na classe de modelos lineares generalizados (McCullagh e Nelder, 1989).

Sob condições gerais de regularidade (ver Sen e Singer, 1993, Capítulo 7), quando o tamanho da amostra é grande,

$$
\left(\begin{array}{l}
\widehat{\beta} \\
\widehat{\phi}
\end{array}\right) \sim \mathcal{N}_{k+1}\left(\left(\begin{array}{l}
\beta \\
\phi
\end{array}\right), K^{-1}\right),
$$

aproximadamente, em que $\widehat{\beta}$ e $\widehat{\phi}$ são os estimadores de máxima verossimilhança de $\beta$ e $\phi$, respectivamente. Assim, é útil obter uma expressão para $K^{-1}$ que pode ser utilizada para obter os erros padrão assintóticos para as estimativas de máxima verossimilhança. Utilizando expressões padrões para inversas de matrizes subdivididas (ver, por exemplo, Rao, 1973, p. 29), obtemos

$$
K^{-1}=K^{-1}(\beta, \phi)=\left(\begin{array}{cc}
K^{\beta \beta} & K^{\beta \phi} \\
K^{\phi \beta} & K^{\phi \phi}
\end{array}\right)
$$

em que

$$
\begin{gathered}
K^{\beta \beta}=\frac{1}{\phi}\left(X^{\top} W X\right)^{-1}\left\{I_{k}+\frac{X^{\top} T c c^{\top} T^{\top} X\left(X^{\top} W X\right)^{-1}}{\gamma \phi}\right\}, \\
\operatorname{com} \gamma=\operatorname{tr}(D)-\phi^{-1} c^{\top} T^{\top} X\left(X^{\top} W X\right)^{-1} X^{\top} T c \\
K^{\beta \phi}=\left(K^{\phi \beta}\right)^{\top}=-\frac{1}{\gamma \phi}\left(X^{\top} W X\right)^{-1} X^{\top} T c,
\end{gathered}
$$

e $K^{\phi \varphi}=\gamma^{-1}$. Aqui $I_{k}$ é a matriz identidade de ordem $k$.

Apresentamos a seguir algumas aproximações para $w_{t}$ e $\mu_{t}^{*}, t=1, \ldots, n$, quando $\mu_{t} \phi$ e $\left(1-\mu_{t}\right) \phi$ são grandes. Em princípio, note que (Abramowitz e Stegun, 1965, pg. 259), com $z \rightarrow \infty$,

$$
\psi(z)=\log (z)-\frac{1}{2 z}-\frac{1}{12 z^{2}}+\frac{1}{120 z^{4}}+\ldots
$$




$$
\psi^{\prime}(z)=\frac{1}{z}+\frac{1}{2 z^{2}}+\frac{1}{6 z^{3}}-\frac{1}{30 z^{5}}+\ldots
$$

No que segue, omitimos o subscrito $t$ (que indexa as observaçôes). Quando $\mu \phi$ e $(1-\mu) \phi$ são grandes, segue de (3.16) que

$$
w \approx \phi\left\{\frac{1}{\mu \phi}+\frac{1}{(1-\mu) \phi}\right\} \frac{1}{g^{\prime}(\mu)^{2}}=\frac{1}{\mu(1-\mu)} \frac{1}{g^{\prime}(\mu)^{2}} .
$$

Também, de (3.15) obtemos

$$
\mu^{*} \approx \log (\mu \phi)-\log ((1-\mu) \phi)=\log \left(\frac{\mu}{1-\mu}\right) .
$$

\subsection{Estimação dos Parâmetros}

Os estimadores de máxima verossimilhança dos parâmetros $\beta$ e $\phi$ são obtidos das equações $U_{\beta}(\beta, \phi)=0$ e $U_{\phi}(\beta, \phi)=0$, não apresentando uma solução analítica em forma fechada. Assim, será necessário obter os estimadores através de maximização numérica do logaritmo da função de verossimilhança, utilizando um processo iterativo, tal como o algoritmo de Newton-Raphson ou escore de Fisher. Outra alternativa é utilizar o algoritmo quasi-Newton, conhecido como método BFGS (este algoritmo foi utilizado nas aplicações descritas no Capítulo 5). Nesta seção descrevemos estes três processos iterativos.

\section{Processo Iterativo de Newton-Raphson}

Seja $\theta=\left(\beta^{\top}, \phi\right)^{\top}$, o vetor de parâmetros e $U(\theta)=\left(U_{\beta}(\beta, \phi)^{\top}, U_{\phi}(\beta, \phi)\right)^{\top}$, o vetor das funções escore de dimensão $(k+1) \times 1$. O processo iterativo de Newton-Raphson para a obtenção da estimativa de máxima verossimilhança do vetor $\theta$ é definido expandindo-se a função escore $U(\theta)$ em torno de um valor inicial $\theta^{(0)}$, tal que

$$
U(\theta) \cong U\left(\theta^{(0)}\right)+U^{\prime}\left(\theta^{(0)}\right)\left(\theta-\theta^{(0)}\right)
$$


em que $U^{\prime}(\theta)$ denota a derivada de $1 \underline{a}$ ordem de $U(\theta)$ com respeito a $\theta^{\top}$. Fazendo $U(\theta)=0$ e repetindo o procedimento acima, chega-se ao seguinte processo iterativo

$$
\theta^{(m+1)}=\theta^{(m)}+\left\{-U^{\prime}\left(\theta^{(m)}\right)\right\}^{-1} U\left(\theta^{(m)}\right), \quad m=0,1, \ldots
$$

O aspecto mais trabalhoso desse esquema iterativo é a inversão da matriz $U^{\prime}(\theta)$.

\section{Processo Iterativo de Escore de Fisher}

A expressão (3.19) apresenta uma forma alternativa equivalente assintoticamente, pois, pela lei dos grandes números, $U^{\prime}(\theta)$ converge para a matriz $K$ definida em (3.13) quando $n \longrightarrow \infty$. Assim, substituindo a informação observada em (3.19) pela esperada, obtemos a seguinte aproximação

$$
\theta^{(m+1)}=\theta^{(m)}+\left\{-K^{(m)}\right\}^{-1} U\left(\theta^{(m)}\right), \quad m=0,1, \ldots
$$

Esse procedimento iterativo é denominado método escore de Fisher. Da mesma forma que o método de Newton-Raphson, o aspecto mais trabalhoso é a inversão da matriz $K$.

\section{Método BFGS}

O método BFGS foi desenvolvido por Broyden, Fletcher, Goldfarb e Shanno (ver Nocedal e Wright, 1999, Capítulo 8). Este utiliza o mesmo princípio do método de Newton-Raphson, diferenciando-se pelo fato de utilizar uma seqüência de matrizes simétricas e positivas definidas $B^{(m)}$ no lugar da matriz $U^{\prime}\left(\theta^{(m)}\right)^{-1}$. Comumente, toma-se como matriz inicial, $B^{(0)}$, a matriz identidade de mesma ordem. A forma recursiva para obter as demais matrizes é dada por

$$
B^{(m+1)}=B^{(m)}-\frac{B^{(m)} s^{(m)}\left(s^{(m)}\right)^{\top} B^{(m)}}{\left(s^{(m)}\right)^{\top} B^{(m)} s^{(m)}}+\frac{y^{(m)}\left(y^{(m)}\right)^{\top}}{\left(y^{(m)}\right)^{\top} s^{(m)}} \quad m=0,1, \ldots
$$

em que $s^{(m)}=\theta^{(m+1)}-\theta^{(m)}$ e $y^{(m)}=U\left(\theta^{(m+1)}\right)-U\left(\theta^{(m)}\right)$. De forma análoga aos 
métodos anteriores, o máximo é obtido pela recorrência

$$
\theta^{(m+1)}=\theta^{(m)}-\alpha^{(m)} B^{(m)} U\left(\theta^{(m)}\right), \quad m=0,1, \ldots,
$$

em que $\alpha^{(m)}$ é um escalar determinado por algum procedimento de busca linear a partir de $\theta^{(m)}$ na direção $-B^{(m)} U\left(\theta^{(m)}\right)$ de forma que $f\left(y ; \theta^{(m)}\right)$ cresça nessa direção.

Esse método está implementado na linguagem de programação ox através da função MaxBFGS. Maiores detalhes sobre esse método podem ser encontrados em Nocedal e Wright (1999, Capítulo 8) e Doornik (2001, Seção 10.2).

Os três procedimentos iterativos são sensíveis à estimativa inicial $\theta^{(0)}$. Ferrari e Cribrari-Neto (2004) sugerem utilizar como uma estimativa para o ponto inicial de $\beta$ a estimativa de mínimos quadrados ordinários desse vetor de parâmetros, obtida de uma regressão linear da resposta transformada $g\left(y_{1}\right), \ldots, g\left(y_{n}\right)$ em $X$, isto é, $\left(X^{\top} X\right)^{-1} X^{\top} z$, em que $z=\left(g\left(y_{1}\right), \ldots, g\left(y_{n}\right)\right)^{\top}$. Em relação ao parâmetro de precisão, eles sugerem um valor inicial para $\phi$ baseado no fato de que $\operatorname{Var}\left(y_{t}\right)=$ $\mu_{t}\left(1-\mu_{t}\right) /(1+\phi)$. De forma alternativa, podemos escrever $\phi=\mu_{t}\left(1-\mu_{t}\right) / \operatorname{Var}\left(y_{t}\right)-1$. Note que

$$
\operatorname{Var}\left\{g\left(y_{t}\right)\right\} \approx \operatorname{Var}\left\{g\left(\mu_{t}\right)+\left(y_{t}-\mu_{t}\right) g^{\prime}\left(\mu_{t}\right)\right\}=\operatorname{Var}\left(y_{t}\right)\left\{g^{\prime}\left(\mu_{t}\right)\right\}^{2}
$$

isto é, $\operatorname{Var}\left(y_{t}\right) \approx \operatorname{Var}\left\{g\left(y_{t}\right)\right\}\left\{g^{\prime}\left(\mu_{t}\right)\right\}^{-2}$. Portanto, o valor inicial sugerido é

$$
\phi^{(0)}=\frac{1}{n} \sum_{t=1}^{n} \frac{\check{\mu}_{t}\left(1-\check{\mu}_{t}\right)}{\check{\sigma}_{t}^{2}}-1,
$$

em que $\check{\mu}_{t}$ é obtido aplicando $g^{-1}(\cdot)$ para o t-ésimo valor ajustado da regressão linear de $g\left(y_{1}\right), \ldots, g\left(y_{n}\right)$ em $X$, isto é,

$$
\check{\mu}_{t}=g^{-1}\left(x_{t}^{\top}\left(X^{\top} X\right)^{-1} X^{\top} z\right) \text { e } \quad \check{\sigma}_{t}^{2}=\check{e}^{\top} \check{e} /\left[(n-k)\left\{g\left(\check{\mu}_{t}\right)\right\}^{2}\right]
$$

Aqui, $\check{e}=z-X\left(X^{\top} X\right)^{-1} X^{\top} z$ é o vetor de resíduos de mínimos quadrados ordinários de uma regressão linear com a variável resposta transformada. Esses valores iniciais 
proporcionaram resultados satisfatórios nas aplicações descritas no Capítulo 5, pois estavam próximos dos valores finais encontrados para as estimativas.

\subsection{Testes de Hipóteses}

Nesta seção apresentamos os testes da razão de verossimilhanças, escore e Wald no contexto do modelo de regressão beta. Adicionalmente aos testes, apresentamos intervalos de confiança para algumas quantidades de interesse.

\subsubsection{Teste da Razão de Verossimilhanças}

É possivel realizar testes assintóticos para fazer inferência sob os parâmetros desconhecidos. Considere, por exemplo, o teste da hipótese nula $\mathcal{H}_{0}: \beta_{1}=\beta_{1}^{(0)}$ versus a hipótese alternativa $\mathcal{H}_{1}: \beta_{1} \neq \beta_{1}^{(0)}$, em que $\beta_{1}=\left(\beta_{1}, \ldots, \beta_{m}\right)^{\top}$ e $\beta_{1}^{(0)}=$ $\left(\beta_{1}^{(0)}, \ldots, \beta_{m}^{(0)}\right)^{\top}$ para $m<k$ e $\beta_{1}^{(0)}$ dado. A estatística da razão de verossimilhanças é

$$
\omega_{1}=2\{\ell(\widehat{\beta}, \widehat{\phi})-\ell(\widetilde{\beta}, \widetilde{\phi})\}
$$

em que $\ell(\beta, \phi)$ é o logaritmo natural da função de verossimilhança e $\left(\widetilde{\beta}^{\top}, \widetilde{\phi}\right)^{\top}$ é o estimador de máxima verossimilhança restrito de $\left(\beta^{\top}, \phi\right)^{\top}$ obtido pela imposição da. hipótese nula. Sob condições gerais de regularidade e sob $\mathcal{H}_{0}, \omega_{1} \stackrel{\mathcal{D}}{\longrightarrow} \chi_{m}^{2}$. Dessa forma o teste pode ser avaliado usando valores críticos aproximados da distribuição $\chi_{m}^{2}$ 


\subsubsection{Teste Escore}

Para descrever o teste escore de $\mathcal{H}_{0}: \beta_{1}=\beta_{1}^{(0)}$ versus $\mathcal{H}_{1}: \beta_{1} \neq \beta_{1}^{(0)}$, considere $U_{1 \beta} u m$ vetor coluna $m$ dimensional contendo os primeiros $m$ elementos da função escore de $\beta$ e $K_{11}^{\beta \beta}$ a matriz $m \times m$ formada das $m$ primeiras linhas e as $m$ primeiras colunas da matriz de $K^{-1}$. Pode-se mostrar, usando (3.8), que $U_{1 \beta}=\phi X_{1}^{\top} T\left(y^{*}-\mu^{*}\right)$, em que $X$ é partida como $\left[X_{1}, X_{2}\right]$ seguindo a partição de $\beta$. A estatística escore de Rao pode ser escrita como

$$
\omega_{2}=\widetilde{U}_{1 \beta}^{\top} \widetilde{K}_{11}^{\beta \beta} \widetilde{U}_{1 \beta}
$$

em que o til indica que as quantidades estão sendo avaliadas no estimador de máxima verossimilhança restrito. Sob condições gerais de regularidade e sob $\mathcal{H}_{0}, \omega_{2} \stackrel{\mathcal{D}}{\longrightarrow} \chi_{m}^{2}$.

\subsubsection{Teste de Wald}

Da mesma forma que os testes anteriores, podemos utilizar o teste de Wald para realizar inferências assintótica acerca do vetor de parâmetros $\beta$. A estatística do teste de $\mathcal{H}_{0}: \beta_{1}=\beta_{1}^{(0)}$ versus $\mathcal{H}_{1}: \beta_{1} \neq \beta_{1}^{(0)}$ é

$$
\omega_{3}=\left(\widehat{\beta}_{1}-\beta_{1}^{(0)}\right)^{\top}\left(\widehat{K}_{11}^{\beta \beta}\right)^{-1}\left(\widehat{\beta}_{1}-\beta_{1}^{(0)}\right)
$$

em que $\widehat{K}_{11}^{\beta \beta}$ é igual a $K_{11}^{\beta \beta}$ avaliado no estimador de máxima verossimilhança irrestrito, e $\widehat{\beta}_{1}$ é o estimador de máxima verossimilhança de $\beta_{1}$. Sob condições gerais de regularidade e sob $\mathcal{H}_{0}, \omega_{3} \stackrel{\mathcal{D}}{\longrightarrow} \chi_{m}^{2}$. Em particular, para testar a significância do $i$-ésimo parâmetro regressor $\left(\beta_{i}\right), i=1, \ldots, k$, podemos utilizar a raiz quadrada sinalizada da estatística de Wald, isto é, $\widehat{\beta}_{i} / \operatorname{se}\left(\widehat{\beta}_{i}\right)$, em que se $\left(\widehat{\beta}_{i}\right)$ é o erro padrão assintótico do estimador de máxima verossimilhança de $\beta_{i}$ obtido da inversa da matriz de informação de Fisher avaliada nos estimadores de máxima verossimilhança. A distribuição nula limite da estatística do teste é normal padrão. 
Adicionalmente aos testes, podemos construir intervalos de confiança para algumas quantidades de interesse. Um intervalo de confiança de aproximadamente $(1-\alpha) \times 100 \%$ para $\beta_{i}, i=1, \ldots, k$ e $0<\alpha<1 / 2$, tem limites dados por

$$
\widehat{\beta}_{i} \pm \Phi^{-1}(1-\alpha / 2) \operatorname{se}\left(\widehat{\beta}_{i}\right)
$$

Similarmente, um intervalo de confiança assintótico $(1-\alpha) \times 100 \%$ para $\phi$ tem limites

$$
\widehat{\phi} \pm \Phi^{-1}(1-\alpha / 2) \operatorname{se}(\widehat{\phi})
$$

em que se $(\widehat{\phi})=\widehat{\gamma}^{-1 / 2}$. Adicionalmente, um intervalo de confiança de aproximadamente $(1-\alpha) \times 100 \%$ para a razão de chances $\exp \left(c \beta_{i}\right)$, quando a ligação logito é utilizada é dado por

$$
\left[\exp \left\{c\left(\widehat{\beta}_{i}-\Phi^{-1}(1-\alpha / 2) \operatorname{se}\left(\widehat{\beta}_{i}\right)\right)\right\}, \quad \exp \left\{c\left(\widehat{\beta}_{i}+\Phi^{-1}(1-\alpha / 2) \operatorname{se}\left(\widehat{\beta}_{i}\right)\right)\right\}\right]
$$

Finalmente, um intervalo de confiança de aproximadamente $(1-\alpha) \times 100 \%$ para $\mu$, a média da variável resposta, para um dado vetor de valores de covariadas $x$, pode ser calculado como

$$
\left[g^{-1}\left(\widehat{\eta}-\Phi^{-1}(1-\alpha / 2) \operatorname{se}(\widehat{\eta})\right), \quad g^{-1}\left(\widehat{\eta}+\Phi^{-1}(1-\alpha / 2) \operatorname{se}(\widehat{\eta})\right)\right]
$$

em que $\widehat{\eta}=x^{\top} \widehat{\beta} \operatorname{ese}(\widehat{\eta})=\sqrt{x^{\top} \widehat{\operatorname{cov}}(\widehat{\beta}) x}$; aqui, $\widehat{\operatorname{cov}}(\widehat{\beta})$ é obtida da inversa da matriz de informação de Fisher avaliada nas estimativas de máxima verossimilhança excluindo a linha e a coluna da matriz correspondente ao parâmetro de dispersão. Os intervalos acima são válidos apenas para funçōes de ligações estritamente crescentes.

\subsection{Técnicas de Diagnóstico}

Uma etapa importante na análise de um ajuste de regressão é a verificação de possíveis afastamentos das suposições feitas para o modelo, especialmente para a 
parte aleatória $\left(y_{t}\right)$ e pelo componente sistemático $\left(\eta_{t}\right)$, bem como a existência de observações extremas com alguma interferência desproporcional nos resultados do ajuste. Tal etapa, conhecida como análise de diagnóstico, tem longa história, e iniciou-se com a análise de resíduos para detectar a presença de pontos extremos e avaliar a adequação da distribuição proposta para a variável resposta. Uma referência importante nesse tópico é o artigo de Cox e Snell (1968) em que é apresentada uma forma bastante geral de definir resíduos usada até os dias atuais. A apresentação desta seção foi baseada em Ferrari e Cribari-Neto (2004) e Paula (2003).

Inicialmente, seräo apresentadas três medidas globais de qualidade do ajuste do modelo e ferramentas gráficas para a detecção de desvios do modelo postulado e observações influentes. Posteriormente, serão apresentados como medidas de análise de influência, a alavanca generalizada proposta por Wei, Hu e Fung (1998) e a distância de Cook (Cook, 1977).

Em princípio, uma medida global da qualidade do ajuste pode ser obtida através do cálculo do "pseudo" $R^{2}\left(R_{p}^{2}\right)$ definido como o quadrado do coeficiente de correlação amostral entre $g(y)$ e $\widehat{\eta}$. Note que $0 \leq R_{p}^{2} \leq 1$ e quanto mais próximo de 1 for o seu valor, melhor será o ajuste.

Mittlböck e Schemper (1996) revisaram algumas medidas da qualidade do ajuste em modelos de regressão logísticos. Utilizaremos duas dessas medidas aqui. A primeira, ainda baseada no pseudo $R^{2}$, é o quadrado do coeficiente de correlação amostral entre $y$ e $\widehat{\mu}\left(g^{-1}(\widehat{\eta})\right)$, que denominaremos por $R_{*}^{2}$. Essa medida apresenta resultados satisfatórios nos estudos realizados por Mittlböck e Schemper (1996). A segunda medida é baseada numa razão de verossimilhanças sendo definida como

$$
R_{L R}^{2}=1-\left[\mathrm{L}_{0} / \widehat{\mathrm{L}}\right]^{2 / n}
$$

em que $\mathrm{L}_{0}$ e $\widehat{\mathrm{L}}$ denotam, respectivamente, as verossimilhanças maximizadas no modelo 
nulo (sem covariáveis) e no modelo sob pesquisa.

\subsubsection{Resíduo Componente do Desvio}

Ferrari e Cribari-Neto (2004) propõem uma medida global de qualidade do ajuste, baseada no fato de que a discrepância de um ajuste pode ser medida como duas vezes a diferença entre o máximo do logaritmo da verossimilhança do modelo saturado e o do modelo sob pesquisa (modelo postulado). Esta medida foi originalmente proposta por Nelder e Wedderburn (1972) no contexto dos modelos lineares generalizados. Seja

$$
D(y ; \mu, \phi)=\sum_{t=1}^{n} 2\left\{\ell_{t}\left(\tilde{\mu}_{t}, \phi\right)-\ell_{t}\left(\mu_{t}, \phi\right)\right\},
$$

em que $\widetilde{\mu}_{t}$ é solução de $\partial \ell_{t} / \partial \mu_{t}=0$, isto é, $\phi\left(y_{t}^{*}-\mu_{t}^{*}\right)=0$. Quando $\phi$ é grande, $\mu_{t}^{*} \approx \log \left\{\mu_{t} /\left(1-\mu_{t}\right)\right\}$ (ver 3.18 ), e então segue que $\widetilde{\mu}_{t} \approx y_{t}$. Para $\phi$ conhecido, podemos definir uma medida de discrepância como $D(y ; \bar{\mu}, \phi)$, em que $\bar{\mu}$ é o estimador de máxima verossimilhança de $\mu$ sob o modelo sendo pesquisado. Quando $\phi$ é desconhecido, uma aproximação para essa quantidade é $D(y ; \widehat{\mu}, \widehat{\phi})$, denominada usualmente de desvio do modelo sob pesquisa. Note que $D(y ; \widehat{\mu}, \widehat{\phi})=\sum_{t=1}^{n}\left(r_{t}^{d}\right)^{2}$, em que

$$
r_{t}^{d}=\operatorname{sinal}\left(y_{t}-\widehat{\mu}_{t}\right)\left\{2\left(\ell_{t}\left(\widetilde{\mu}_{t}, \widehat{\phi}\right)-\ell_{t}\left(\widehat{\mu}_{t}, \widehat{\phi}\right)\right)\right\}^{1 / 2}
$$

Note que a $t$-ésima observação contribui com a quantidade $\left(r_{t}^{d}\right)^{2}$ para o desvio e uma observação com um valor absoluto grande de $r_{t}^{d}$ pode ser vista como discrepante.

O resíduo ordinário padronizado é definido como

$$
r_{t}=\frac{y_{t}-\widehat{\mu}_{t}}{\sqrt{\widehat{\operatorname{Var}}\left(y_{t}\right)}}
$$

em que $\widehat{\mu}_{t}=g^{-1}\left(x_{t}^{\top} \widehat{\beta}\right)$ e $\widehat{\operatorname{Var}}\left(y_{t}\right)=\left\{\widehat{\mu}_{t}\left(1-\widehat{\mu}_{t}\right)\right\} /(1+\widehat{\phi})$. Um gráfico desses residuos contra a ordem das observaçōes $(t)$ não deveria mostrar nenhuma tendência. Através 
desse gráfico é possível verificar se existem pontos suspeitos de serem aberrantes. Um outro gráfico importante é o de $r_{t}$ contra $\widehat{\eta}_{t}$ que é utilizado para verificar se a função de ligação está mal especificada caso este gráfico apresente alguma tendência.

\subsubsection{Gráficos de Probabilidade Meio-Normal com Envelopes}

Como a distribuição dos resíduos não é conhecida, gráficos de probabilidade meionormal com envelopes simulados são ferramentas de diagnóstico muito úteis (Atkinson, 1985, Seção 4.2; Neter et al., 1996, Seção 14.6). A idéia é acrescentar ao gráfico meio-normal usual um envelope simulado que pode ser usado para decidir se as respostas observadas são consistentes com o modelo ajustado. Apresentamos a seguir os passos para a construção do gráfico meio-normal com envelope simulado:

1. ajuste o modelo e gere uma amostra simulada de $n$ observações independentes utilizando o modelo ajustado como se este fosse o modelo verdadeiro;

2. ajuste o modelo para a amostra gerada, e calcule os valores absolutos ordenados da medida de diagnóstico de interesse;

3. repita os passos (1) e (2) $k$ vezes;

4. considere os $n$ grupos de $k$ estatísticas de ordem; para cada grupo, calcule suas respectivas médias, valores mínimos e máximos; e

5. construa o gráfico desses valores e da medida de diagnóstico ordenada da amostra original contra os escores meio-normais $\Phi^{-1}((t+n-1 / 8) /(2 n+1 / 2))$, em que $\Phi(\cdot)$ é a função de distribuição acumulada da distribuição normal padrão. 
Os valores mínimos e máximos das $k$ estatísticas de ordem produzem o envelope. A sugestão de Atkinson (1985, p. 36) é usar $k=19$. Desse modo, a probabilidade do maior resíduo de um envelope particular exceder o limite superior fica sendo aproximadamente igual a $1 / 20=0,05$. As correspondentes observações dos resíduos absolutos que se encontram fora dos limites fornecidos pelo envelope simulado, merecem uma pesquisa adicional. Tendências não aleatórias dos resíduos dentro do envelope gerado podem indicar especificação incorreta da distribuição dos dados, da parte sistemática do modelo ou da função de ligação.

\subsubsection{Leverage Generalizado}

Leverage é um componente chave na análise de influência em modelos de regressão. Usualmente, é medido pelos elementos $h_{i j}$ da matriz $H$ que é conhecida como matriz de projeção ou "matriz chapéu" (ver, por exemplo, Chatterjee e Hadi, 1986; Cook e Weisberg, 1982, pg. 15, 142) e é usado para avaliar a importância individual de cada observação no próprio valor ajustado. Na regressão linear múltipla, por exemplo, é muito razoável utilizar $h_{i i}$ como uma medida da influência da $i$-ésima observação sobre o próprio valor ajustado. Supondo que todos os pontos exerçam a mesma influência sobre os valores ajustados, pode-se esperar que $h_{i i}$ esteja próximo $\operatorname{de} \operatorname{tr}(H) / n=k / n$. Uma sugestão é examinar aqueles pontos tais que $h_{i i} \geq 2 k / n$, que são conhecidos como pontos de alavanca ou de leverage grandes. Recentemente, Wei, Hu e Fung (1998) generalizaram a definição de pontos de alavanca para modelos bastante gerais cuja variável resposta seja contínua. Nessa generalização incluem-se outros métodos de estimação, além de máxima verossimilhança, e outros enfoques tais como enfoque Bayesiano. 
O leverage generalizado proposto por Wei, Hu e Fung (1998) é definido como

$$
\operatorname{GL}(\widetilde{\theta})=\frac{\partial \widetilde{y}}{\partial y^{\top}}=\left(\frac{\partial \widetilde{y}_{t}}{\partial y_{u}}\right)_{n \times n}
$$

em que $\theta$ é um vetor $s$-dimensional tal que $\mathrm{E}(y)=\mu(\theta)$ e $\tilde{\theta}$ é um estimador de $\theta$, com $\widetilde{y}=\mu(\widetilde{\theta})$. Por esta definição, podemos observar que o leverage generalizado para a t-ésima observação é a razão instantânea de mudança do t-ésimo valor predito em relação ao u-ésimo valor da resposta. Assim, essa medida de influência das observações é definida a partir do modelo ajustado. Wei, Hu, e Fung (1998) afirmam que observaçôes com $\partial \widetilde{y}_{t} / \partial y_{t}=\mathrm{GL}_{t t}$ grandes são pontos de alavanca. Os autores também notaram que o leverage generalizado $\operatorname{GL}(\widetilde{\theta})$ é invariante sob reparametrizações, isto é, se $\theta=\theta(\tilde{\delta})$ é uma transformação um a um e $\widetilde{\delta}=\theta^{-1}(\widetilde{\theta})$, então $\operatorname{GL}(\widetilde{\delta})=\operatorname{GL}(\widetilde{\theta})$.

Seja $\widehat{\theta}$ o estimador de máxima verossimilhança de $\theta$, assumindo que exista e seja único, e assuma que o logaritmo da função de verossimilhança tem derivadas contínuas de segunda ordem com respeito a $\theta$ e a $y$. Wei, Hu e Fung (1998) mostraram que o leverage generalizado é dado por

$$
\mathrm{GL}(\theta)=D_{\theta}\left(-\frac{\partial^{2} \ell}{\partial \theta \partial \theta^{\top}}\right)^{-1} \frac{\partial^{2} \ell}{\partial \theta \partial y^{\top}}
$$

avaliado em $\widehat{\theta}$, em que $D_{\theta}=\partial \mu / \partial \theta^{\top}$.

Apresentamos a seguir o cálculo do leverage generalizado no modelo de regressão beta sob dois enfoques: primeiramente sob a suposição de que o parâmetro de precisão $\phi$ é conhecido e, num segundo momento, admitindo que $\phi$ é desconhecido.

Considerando $\phi$ conhecido, Ferrari e Cribari-Neto (2004) obtiveram uma expressão de forma fechada para $G L(\beta)$ no modelo de regressão beta proposto na Seção 3.2. O leverage generalizado fica dado por

$$
\mathrm{GL}(\beta)=D_{\beta}\left(-\frac{\partial^{2} \ell}{\partial \beta \partial \beta^{\top}}\right)^{-1} \frac{\partial^{2} \ell}{\partial \beta \partial y^{\top}},
$$


em que

$$
D_{\beta}=\frac{\partial \mu}{\partial \beta^{\top}}=\frac{\partial \mu}{\partial \eta^{\top}} \frac{\partial \eta}{\partial \beta^{\top}}=T X
$$

e de (3.12)

$$
\begin{aligned}
-\frac{\partial^{2} \ell}{\partial \beta_{i} \partial \beta_{j}} & =\sum_{t=1}^{n}\left\{\frac{\partial^{2} \ell_{t}\left(\mu_{t}, \phi\right)}{\partial \mu_{t}^{2}} \frac{\mathrm{d} \mu_{t}}{\mathrm{~d} \eta_{t}}+\frac{\partial \ell_{t}\left(\mu_{t}, \phi\right)}{\partial \mu_{t}} \frac{\partial}{\partial \mu_{t}}\left(\frac{\mathrm{d} \mu_{t}}{\mathrm{~d} \eta_{t}}\right)\right\} \frac{\mathrm{d} \mu_{t}}{\mathrm{~d} \eta_{t}} x_{t i} x_{t j} \\
& =-\phi \sum_{i=1}^{n}\left\{\phi\left\{\psi^{\prime}\left(\mu_{t} \phi\right)+\psi^{\prime}\left(\left(1-\mu_{t}\right) \phi\right)\right\}+\left(y_{t}^{*}-\mu_{t}^{*}\right) \frac{g^{\prime \prime}\left(\mu_{t}\right)}{g^{\prime}\left(\mu_{t}\right)}\right\} \frac{x_{t i} x_{t j}}{\left\{g^{\prime}\left(\mu_{t}\right)\right\}^{2}}
\end{aligned}
$$

que, em forma matricial, é dado por

$$
-\frac{\partial^{2} \ell}{\partial \beta \partial \beta^{\top}}=\phi X^{\top} Q X
$$

em que $Q=\operatorname{diag}\left\{q_{1}, \ldots, q_{n}\right\}$ com

$$
q_{t}=\left\{\phi\left\{\psi^{\prime}\left(\mu_{t} \phi\right)+\psi^{\prime}\left(\left(1-\mu_{t}\right) \phi\right)\right\}+\left(y_{t}^{*}-\mu_{t}^{*}\right) \frac{g^{\prime \prime}\left(\mu_{t}\right)}{g^{\prime}\left(\mu_{t}\right)}\right\} \frac{1}{\left\{g^{\prime}\left(\mu_{t}\right)\right\}^{2}}, \quad t=1, \ldots, n
$$

Adicionalmente, temos que

$$
\frac{\partial^{2} \ell}{\partial \beta_{i} y_{t}}=\phi \sum_{t=1}^{n}\left(\frac{1}{y_{t}\left(1-y_{t}\right)} \frac{1}{g^{\prime}\left(\mu_{t}\right)} x_{t i}\right),
$$

que, em forma matricial, fica

$$
\frac{\partial^{2} \ell}{\partial \beta \partial y^{\top}}=\phi X^{\top} T M
$$

em que $M=\operatorname{diag}\left\{m_{1}, \ldots, m_{n}\right\} \operatorname{com} m_{t}=1 /\left\{y_{t}\left(1-y_{t}\right)\right\}, t=1, \ldots, n$. Finalmente, (3.22) pode ser escrito como

$$
G L(\beta)=T X\left(X^{\top} Q X\right)^{-1} X^{\top} T M
$$

Ferrari e Cribari-Neto (2004) notaram que ao substituir a informaçāo observada, $-\partial^{2} \ell / \partial \beta \partial \beta^{\top}$, pela informação esperada, $\mathrm{E}\left(-\partial^{2} \ell / \partial \beta \partial \beta^{\top}\right)$, a expressão para $\mathrm{GL}(\beta)$ 
fica como dada em (3.23) mas com a matriz $Q$ substituída por $W$, sendo dada por $\mathrm{GL}^{*}(\beta)$. Pode-se notar que os elementos da diagonal de $\mathrm{GL}^{*}(\beta)$ são os mesmos de

$$
M^{1 / 2} T X\left(X^{\top} W X\right)^{-1} X^{\top} T M^{1 / 2}
$$

e que $M^{1 / 2} T$ é uma matriz diagonal cujo t-ésimo elemento diagonal é dado por $\left\{g^{\prime}\left(\mu_{t}\right) V\left(y_{t}\right)^{1 / 2}\right\}^{-1}$. Outro fato importante aqui é notar que existe uma conexão estreita entre os elementos da diagonal de $\mathrm{GL}^{*}(\beta)$ e aqueles da "matriz chapéu" usual de modelos lineares generalizados,

$$
H=W^{1 / 2} X\left(X^{\top} W X\right)^{-1} X^{\top} W^{1 / 2},
$$

quando $\phi$ é grande. A relação origina-se do fato de que, quando o parâmetro de precisão é grande, o t-ésimo elemento da diagonal de $W^{1 / 2}$ é aproximadamente igual a

$$
\left[g^{\prime}\left(\mu_{t}\right) V\left(\mu_{t}\right)^{1 / 2}\right]^{-1}
$$

como pode ser verificado na expressão (3.17).

Agora, considerando $\phi$ desconhecido, temos que $\theta^{\top}=\left(\beta^{\top}, \phi\right)$. Segue, então, que,

$$
D_{\theta}=\left[\begin{array}{ll}
\frac{\partial \mu}{\partial \beta^{\top}} & \frac{\partial \mu}{\partial \phi}
\end{array}\right]=\left[\begin{array}{ll}
T X & 0
\end{array}\right]
$$

em que 0 é um vetor $n$-dimensional de zeros. A quantidade $-\partial^{2} \ell / \partial \theta \partial \theta^{\top}$ é dada por (3.13) com $W$ substituído por $Q$ e c substituído por $f$, em que $f=\left(f_{1}, \ldots, f_{n}\right)^{\top}$ com $f_{t}=\left\{c_{t}-\left(y_{t}^{*}-\mu_{t}^{*}\right)\right\}, t=1, \ldots, n$. Assim, a inversa de $-\partial^{2} \ell / \partial \theta \partial \theta^{\top}$ será dada por (3.14) com $W$ e $c$ substituídos por $Q$ e $f$, respectivamente. Adicionalmente,

$$
\frac{\partial^{2} \ell}{\partial \theta \partial y^{\top}}=\left(\begin{array}{c}
\phi X^{\top} T M \\
b^{\top}
\end{array}\right) \text {, }
$$

em que $b=\left(b_{1}, \ldots, b_{n}\right)^{\top}$ com $b_{t}=-\left(y_{t}-\mu_{t}\right) /\left\{y_{t}\left(1-y_{t}\right)\right\}, t=1, \ldots, n$. Finalmente, o leverage generalizado é dado por

$$
\mathrm{GL}(\beta, \phi)=\mathrm{GL}(\beta)+\frac{1}{\gamma \phi} T X\left(X^{\top} Q X\right)^{-1} X^{\top} T f\left(f^{\top} T X\left(X^{\top} Q X\right)^{-1} X^{\top} T M-b^{\top}\right)
$$


em que $\mathrm{GL}(\beta)$ é dado em (3.23). Quando $\phi$ é grande, $\operatorname{GL}(\beta, \phi) \approx \mathrm{GL}(\beta)$.

\subsubsection{Influência}

Um outro tópico importante na análise de diagnóstico é a detecção de observações influentes, isto é, pontos que exercem um peso desproporcional nas estimativas dos parâmetros do modelo. Uma medida de influência de cada observação nas estimativas dos parâmetros de regressão é a distância de Cook (Cook, 1977) dada por

$$
k^{-1}\left(\widehat{\beta}-\widehat{\beta}_{(t)}\right)^{\top} X^{\top} W X\left(\widehat{\beta}-\widehat{\beta}_{(t)}\right),
$$

em que $\widehat{\beta}_{(t)}$, é a estimativa do parâmetro sem a $t$-ésima observação. Essa quantidade mede a distância quadrática entre $\widehat{\beta}$ e $\widehat{\beta}_{(t)}$. Para evitar ajustar o modelo $n+1$ vezes, utilizamos a aproximação usual para a distância de Cook dada por

$$
C_{t}=\frac{h_{t t} r_{t}^{2}}{k\left(1-h_{t t}\right)^{2}}
$$

que combina alavanca (leverage) e resíduos. Portanto $C_{t}$ será grande quando o t-ésimo ponto fornecer $r_{t}$ grande ou quando $h_{t t}$ for próximo de um. Uma prática comum é construir um gráfico de $C_{t}$ contra a ordem das observações $(t)$ para detectar pontos suspeitos de serem influentes. 
Capítulo 4

\section{Avaliação de Resultados}

\section{Assintóticos em Amostras Finitas}

\subsection{Introdução}

Neste capítulo avaliamos numericamente a qualidade das aproximações utilizadas nas inferências sobre os parâmetros do modelo de regressão beta em amostras finitas. O interesse é avaliar o viés do estimador de máxima verossimilhança, a proximidade de sua distribuição à distribuição normal de referência, a distorção de tamanho dos testes da razão de verossimilhanças, escore e Wald e também verificar a qualidade da aproximação das distribuições das estatísticas dos testes por uma distribuição qui-quadrado adequada. Todos os resultados foram obtidos por simulação de Monte Carlo. 


\subsection{Detalhes Metodológicos}

As simulações computacionais deste trabalho foram realizadas usando a versão 3.30 da linguagem de programação matricial ox. Esta linguagem de programação dispõe de três geradores de números aleatórios denominados:

- 'PM' - Park e Miller modificado, de período aproximadamente $2^{32}$ e que exige uma semente;

- 'GM' - George Marsaglia, de período aproximadamente $2^{60}$ e que exige duas sementes;

- 'LE' - Pierre L'Ecuyer, de período aproximadamente $2^{113}$ e que exige quatro sementes.

Nas simulações deste capítulo utilizamos o gerador 'GM' - George Marsaglia. Maiores detalhes sobre estes geradores de números aleatórios podem ser encontrados em Doornik (2001).

Os resultados numéricos apresentados a seguir baseiam-se no modelo de regressão beta com estrutura

$$
g\left(\mu_{t}\right)=\beta_{0}+\beta_{1} x_{t 1}+\beta_{2} x_{t 2}+\beta_{3} x_{t 3}+\beta_{4} x_{t 4}, \quad t=1, \ldots, n
$$

com $g$ sendo a função de ligação logito (ver Seção 3.3).

Os passos para a simulação de Monte Carlo são descritos a seguir. Definimos valores verdadeiros para os parâmetros de regressão $\left(\beta_{0}=1, \beta_{1}=0, \beta_{2}=0, \beta_{3}=\right.$ 1. $\beta_{4}=1$ ) que permanecem fixos para todas as simulações. Para o parámetro de precisão são atribuídos dois valores: $\phi=120$ e $\phi=20$. A matriz regressora $X$ é gerada das distribuições $\mathcal{U}(0,1), \mathcal{N}(0,1), t_{4}$ e $\operatorname{Exp}(2)$. Os valores de $X$ permanecem 
constantes em todo o experimento. Consideramos os tamanhos amostrais $n=20$, $n=30$ e $n=50$. O número de replicações de Monte Carlo foi fixado em 10000 .

Para cada réplica de Monte Carlo geramos uma amostra aleatória da variável resposta $y_{1}, \ldots, y_{n}, \operatorname{com} y_{t} \sim \operatorname{Beta}\left(\mu_{t}, \phi\right)$ e

$$
\mu_{t}=\frac{\exp \left(\beta_{0}+\beta_{1} x_{t 1}+\beta_{2} x_{t 2}+\beta_{3} x_{t 3}+\beta_{4} x_{t 4}\right)}{1+\exp \left(\beta_{0}+\beta_{1} x_{t 1}+\beta_{2} x_{t 2}+\beta_{3} x_{t 3}+\beta_{4} x_{t 4}\right)}
$$

para $t=1, \ldots, n$. Dados os valores do vetor de resposta e a matriz de regressores, avaliamos a regressão auxiliar

$$
\left(X^{\top} X\right)^{-1} X^{\top} z
$$

em que $z$ é o vetor da variável resposta transformada $z=\left(g\left(y_{1}\right), \ldots, g\left(y_{n}\right)\right)^{\top}$. As estimativas obtidas dessa regressão auxiliar foram tomadas como pontos iniciais para o processo de maximização do logaritmo da função de verossimilhança (3.4). Para a maximização, utilizamos o algoritmo quasi-Newton BFGS, através da função MaxBFGS, que está implementado na linguagem de programação ox. Além disso, para cada réplica de Monte Carlo, seguindo o mesmo procedimento acima, foram calculadas as estimativas dos parâmetros restritas à hipótese $\mathcal{H}_{0}:\left(\beta_{1}, \beta_{2}\right)=(0,0)$, a fim de calcularmos as estatísticas dos testes.

\subsection{Comportamento dos Estimadores}

Nesta seção apresentamos os resultados de simulação referentes ao comportamento dos estimadores de máxima verossimilhança dos parâmetros do modelo de regressão beta (4.1). A Tabela 4.1 apresenta algumas quantidades relativas às estimativas dos parâmetros quando estes têm valores verdadeiros dados por $\beta_{0}=1, \beta_{1}=0, \beta_{2}=$ $0, \beta_{3}=1, \beta_{4}=1, \phi=120$ e a matriz de regressores $X$ gerada de uma distribuição 
$\mathcal{U}(0,1)$. Nas colunas da Tabela 4.1 são apresentadas as seguintes quantidades: média, desvio padrão, viés, raiz quadrada do erro quadrático médio, coeficientes de assimetria e curtose, taxa de cobertura e média dos limites inferiores e superiores dos intervalos de confiança, construídos com um coeficiente de confiança de 95\%. Para cada réplica de Monte Carlo, foi calculado o intervalo de confiança para cada parâmetro e verificado se o verdadeiro valor do parâmetro pertence ou não a este intervalo. A porcentagem das réplicas em que o verdadeiro valor do parâmetro pertence ao intervalo representa a taxa de cobertura. Como os intervalos foram construídos com um coeficiente de confiança de $95 \%$, espera-se que a taxa de cobertura esteja próxima deste valor.

Observa-se na Tabela 4.1 que as estimativas do desvio padrão, viés e raiz quadrada do erro quadrático médio de todos os estimadores dos parâmetros diminuem com o aumento do tamanho da amostra, como era de se esperar. Nota-se que o viés dos estimadores dos parâmetros de regressão está próximo de zero, apresentando uma diferença em relação aos verdadeiros valores na terceira casa decimal, com exceção do estimador de $\beta_{4}$ quando o tamanho da amostra é $n=20$. No entanto, para o parâmetro de precisão $\phi$ não obtivemos resultados tão satisfatórios. Por exemplo, quando o tamanho amostral é $n=20$, temos um viés positivo em torno de 64 unidades, o que dá um viés relativo de aproximadamente $54 \%$. Para $n=50$ o viés é reduzido, porém ainda é de 19 unidades, fornecendo um viés relativo de aproximadamente $16 \%$.

Em relação ao comportamento assintótico, espera-se que cada estimador dos parâmetros do modelo tenha uma distribuição normal, ou seja, que as estimativas dos coeficientes de assimetria e curtose estejam em torno de zero. Se o coeficiente de assimetria for positivo, a distribuição é assimétrica à direita e, caso contrário, será assimetria à esquerda. Os parâmetros de regressão apresentam as estimativas desses 


\section{Tabela 4.1}

Medidas amostrais dos estimadores dos parâmetros de regressão e precisão, $\theta=\left(\beta_{0}, \beta_{1}, \beta_{2}, \beta_{3}, \beta_{4}, \phi\right)^{\top}=(1,0,0,1,1,120)^{\top}$; covariáveis uniformes.

\begin{tabular}{ccccccccccc}
\hline \hline$n$ & Est. & Média & DP & Viés & REQM & Assim. & Curt. & TCob. & LI & LS \\
\hline \multirow{6}{*}{20} & $\widehat{\beta}_{0}$ & 0,997 & 0,221 & $-0,003$ & 0,22 & 0,08 & 0,08 & 93,5 & 0,626 & 1,368 \\
& $\widehat{\beta}_{1}$ & 0,002 & 0,229 & 0,002 & 0,23 & 0,02 & 0,01 & 92,0 & $-0,383$ & 0,388 \\
& $\widehat{\beta}_{2}$ & $-0,003$ & 0,236 & $-0,003$ & 0,24 & $-0,03$ & $-0,03$ & 92,5 & $-0,400$ & 0,394 \\
& $\widehat{\beta}_{3}$ & 1,008 & 0,284 & 0,008 & 0,28 & 0,03 & $-0,02$ & 92,0 & 0,537 & 1,480 \\
& $\widehat{\beta}_{4}$ & 1,011 & 0,286 & 0,011 & 0,29 & 0,03 & 0,00 & 92,2 & 0,533 & 1,489 \\
& $\hat{\phi}$ & 184,5 & 80,7 & 64,5 & 103,3 & 2,24 & 10,58 & 93,2 & 70,1 & 298,8 \\
\hline \hline \multirow{6}{*}{30} & $\widehat{\beta}_{0}$ & 1,002 & 0,167 & 0,002 & 0,17 & 0,01 & 0,11 & 94,6 & 0,706 & 1,299 \\
& $\widehat{\beta}_{1}$ & $-0,002$ & 0,167 & $-0,002$ & 0,17 & 0,00 & 0,00 & 93,0 & $-0,296$ & 0,292 \\
& $\widehat{\beta}_{2}$ & 0,000 & 0,170 & 0,000 & 0,17 & 0,03 & 0,01 & 93,1 & $-0,299$ & 0,300 \\
& $\widehat{\beta}_{3}$ & 1,002 & 0,197 & 0,002 & 0,20 & 0,00 & $-0,00$ & 93,6 & 0,653 & 1,351 \\
& $\widehat{\beta}_{4}$ & 1,003 & 0,189 & 0,003 & 0,19 & 0,00 & $-0,00$ & 93,3 & 0,669 & 1,337 \\
& $\hat{\phi}$ & 157,1 & 49,1 & 37,1 & 61,6 & 1,46 & 4,75 & 93,4 & 77,6 & 236,6 \\
\hline \hline & $\widehat{\beta}_{0}$ & 1,001 & 0,136 & 0,001 & 0,14 & $-0,01$ & $-0,05$ & 95,7 & 0,750 & 1,252 \\
& $\widehat{\beta}_{1}$ & $-0,001$ & 0,131 & $-0,001$ & 0,13 & 0,02 & 0,02 & 94,1 & $-0,239$ & 0,237 \\
50 & $\widehat{\beta}_{2}$ & $-0,001$ & 0,142 & $-0,001$ & 0,14 & 0,02 & $-0,00$ & 94,1 & $-0,262$ & 0,260 \\
& $\widehat{\beta}_{3}$ & 1,003 & 0,151 & 0,003 & 0,15 & 0,01 & $-0,05$ & 94,7 & 0,724 & 1,281 \\
& $\widehat{\beta}_{4}$ & 1,003 & 0,143 & 0,003 & 0,14 & 0,03 & 0,04 & 94,3 & 0,740 & 1,266 \\
& $\hat{\phi}$ & 139,7 & 30,9 & 19,7 & 36,7 & 0,93 & 1,54 & 94,3 & 84,9 & 194,5 \\
\hline \hline & & & & & & & & & &
\end{tabular}

coeficientes próximos de zero, mesmo para um tamanho amostral pequeno $(n=20)$. Novamente, a estimação do parâmetro de precisão $\phi$ não apresenta um resultado satisfatório. Para $n=20$, as estimativas dos coeficientes estão acima de 2 unidades para a assimetria e 10 unidades para a curtose. Esse fato é amenizado quando o tamanho da amostra aumenta $(n=50)$, onde podemos notar que as estimativas dos coeficientes de assimetria e curtose para o estimador do parâmetro de precisão são 0,93 e 1,54 , respectivamente. Como a estimativa do coeficiente de assimetria para o 
estimador do parâmetro $\phi$ é sempre positivo, sua distribuição é assimétrica à direita.

Ainda da Tabela 4.1, podemos observar que as taxas de cobertura de todos os intervalos de confiança dos parâmetros do modelo de regressão beta estão razoavelmente próximas do coeficiente nominal (95\%). Para $n=20$, os valores encontrados estão em torno de $92 \%$ e $93 \%$, chegando a $94 \%$ e $95 \%$ para $n=50$. Outro fato interessante é que os intervalos de confiança para todos os parâmetros de regressão são, em média, simétricos em torno dos verdadeiros valores dos parâmetros, independente do tamanho da amostra. O contrário ocorre com o intervalo de confiança para o parâmetro de precisão, este sendo assimétrico à direita, como observado na análise do comportamento assintótico da distribuição desse parâmetro. Além disso, com o aumento do tamanho da amostra, temos intervalos de amplitude média menor para todos os parâmetros, como era de se esperar. Por exemplo, o valor da amplitude média para o parâmetro $\phi$ quando $n=20$ é de 228,7 unidades, enquanto que para $n=50$ este, valor reduz-se para 109, 6 unidades.

Com o objetivo de verificar se o valor do parâmetro de precisão $\phi$ influencia na estimação dos parâmetros de regressão, realizamos as mesmas simulações atribuindo os mesmos valores para os parâmetros de regressão, mas modificando o valor do parâmetro $\phi$ de 120 para um valor menor de 20 unidades. Nessa situação, a dispersão dos dados é maior do que no primeiro estudo. Os resultados dessas simulações encontram-se na Tabela 4.2.

Verifica-se que os vieses dos estimadores dos parâmetros de regressão continuam próximos de zero, indicando uma influência pequena do valor do parâmetro de precisão. O viés relativo do parâmetro $\phi$ foi aproximadamente de $58 \%$ para $n=20$. Sua estimativa foi 31,6 , enquanto se esperava 20.

Com relação ao comportamento assintótico, os parâmetros de regressão apresen- 
Tabela 4.2

Medidas amostrais dos estimadores dos parâmetros de regressão e precisão, $\theta=\left(\beta_{0}, \beta_{1}, \beta_{2}, \beta_{3}, \beta_{4}, \phi\right)^{\top}=(1,0,0,1,1,20)^{\top} ;$ covariáveis uniformes.

\begin{tabular}{ccccccccccc}
\hline \hline$n$ & Est. & Média & DP & Viés & REQM & Assim. & Curt. & TCob. & LI & LS \\
\hline \multirow{6}{*}{20} & $\widehat{\beta}_{0}$ & 1,010 & 0,533 & 0,010 & 0,53 & 0,08 & 0,14 & 92,8 & 0,153 & 1,867 \\
& $\widehat{\beta}_{1}$ & 0,001 & 0,549 & 0,001 & 0,55 & 0,06 & 0,03 & 91,4 & $-0,883$ & 0,884 \\
& $\widehat{\beta}_{2}$ & $-0,008$ & 0,562 & $-0,008$ & 0,56 & $-0,03$ & 0,07 & 91,7 & $-0,921$ & 0,904 \\
& $\widehat{\beta}_{3}$ & 1,030 & 0,673 & 0,030 & 0,67 & 0,07 & 0,10 & 91,4 & $-0,047$ & 2,107 \\
& $\widehat{\beta}_{4}$ & 1,047 & 0,678 & 0,047 & 0,68 & 0,03 & 0,08 & 92,0 & $-0,049$ & 2,143 \\
& $\widehat{\phi}$ & 31,6 & 14,1 & 11,6 & 18,3 & 2,96 & 27,45 & 92,7 & 11,9 & 51,3 \\
\hline \hline \multirow{6}{*}{30} & $\widehat{\beta}_{0}$ & 1,003 & 0,393 & 0,003 & 0,39 & 0,02 & $-0,02$ & 94,5 & 0,317 & 1,689 \\
& $\widehat{\beta}_{1}$ & $-0,003$ & 0,387 & $-0,003$ & 0,39 & 0,02 & 0,05 & 93,4 & $-0,679$ & 0,672 \\
& $\widehat{\beta}_{2}$ & 0,000 & 0,397 & 0,000 & 0,40 & $-0,00$ & $-0,03$ & 92,9 & $-0,689$ & 0,689 \\
& $\widehat{\beta}_{3}$ & 1,023 & 0,459 & 0,023 & 0,46 & 0,04 & 0,06 & 93,0 & 0,221 & 1,824 \\
& $\widehat{\beta}_{4}$ & 1,031 & 0,446 & 0,031 & 0,45 & 0,00 & $-0,03$ & 92,6 & 0,262 & 1,801 \\
& $\widehat{\phi}^{2}$ & 26,4 & 8,1 & 6,4 & 10,3 & 1,24 & 2,67 & 93,5 & 12,9 & 39,8 \\
\hline \hline & $\widehat{\beta}_{0}$ & 1,007 & 0,315 & 0,007 & 0,32 & $-0,03$ & 0,09 & 95,3 & 0,435 & 1,579 \\
& $\widehat{\beta}_{1}$ & 0,001 & 0,296 & 0,001 & 0,30 & 0,01 & 0,14 & 94,2 & $-0,541$ & 0,543 \\
50 & $\widehat{\beta}_{2}$ & $-0,006$ & 0,325 & $-0,006$ & 0,33 & $-0,01$ & 0,03 & 94,5 & $-0,602$ & 0,590 \\
& $\widehat{\beta}_{3}$ & 1,013 & 0,349 & 0,013 & 0,35 & 0,06 & 0,08 & 93,6 & 0,378 & 1,648 \\
& $\widehat{\beta}_{4}$ & 1,013 & 0,330 & 0,013 & 0,33 & 0,00 & 0,03 & 93,6 & 0,417 & 1,609 \\
& $\hat{\phi}$ & 23,5 & 5,3 & 3,5 & 6,3 & 0,91 & 1,55 & 94,0 & 14,2 & 32,7 \\
\hline \hline
\end{tabular}

tam novamente valores dos coeficientes de assimetria e curtose em torno de zero, para todos os tamanhos amostrais considerados. Da mesma forma que para o caso em que $\phi=120$, os coeficientes de assimetria e curtose do estimador do parâmetro de precisão $\phi$ não são próximos de zero. Por exemplo, para $n=20$, os valores desses coeficientes são 2,96 e 27,45 , respectivamente. Verifica-se novamente que o valor do coeficiente de assimetria para o estimador do parâmetro $\phi$ é sempre positivo, caracterizando uma distribuç̧ão assimétrica à direita. 
Nota-se ainda que as taxas de cobertura dos intervalos de confiança de todos os parâmetros se mantiveram praticamente as mesmas observadas no estudo anterior. $O$ mesmo não se pode dizer em relação às amplitudes médias dos intervalos de confiança, que agora são maiores. Esse fato pode ser explicado pelo valor pequeno considerado para o parâmetro $\phi$, que leva a uma maior variabilidade da variável resposta e, conseqüentemente, maior incerteza acerca dos parâmetros do modelo.

De posse dos comentários apresentados para as Tabelas 4.1 e 4.2, podemos concluir que o valor do parâmetro de precisão influencia na estimação intervalar dos parâmetros de regressão, mas tem pouco efeito sobre a estimação pontual.

Outra característica do modelo que pode ter impacto nas estimação dos parâmetros é a matriz regressora $X$. A Tabela 4.3 apresenta resultados de simulação para os estimadores dos parâmetros do modelo, quando a matriz regressora é gerada das seguintes distribuições: $\mathcal{N}(0,1), t_{4}$ e $\operatorname{Exp}(2)$. Vale lembrar que nos dois estudos anteriores $X$ foi obtida de uma distribuição $\mathcal{U}(0,1)$. Escolhemos a distribuição $t$-Student por ter caudas pesadas e a distribuição exponencial por ser de forma assimétrica à direita. Os valores gerados foram padronizados de tal forma que cada variável tivesse média 0 e variância 1. O tamanho da amostra foi fixado em $n=20$ e os valores dos parâmetros são: $\beta_{0}=1, \beta_{1}=0, \beta_{2}=0, \beta_{3}=1, \beta_{4}=1, \phi=20$. Note que a simulação foi realizada em uma situação extrema, pois o tamanho da amostra e o valor verdadeiro do parâmetro de precisão são ambos pequenos.

Para as três distribuições consideradas na Tabela 4.3, observa-se que os vieses dos estimadores dos parâmetros de regressão estão próximos de zero. O parâmetro de precisão, quando a matriz regressora é obtida das distribuições $\mathcal{N}(0,1), t_{4} \operatorname{e} \operatorname{Exp}(2)$, é superestimado em $58 \%, 56 \%$ e 55\%, respectivamente. Em relação à aproximação das distribuições dos estimadores dos parâmetros pela distribuição normal, verifica-se 
Tabela 4.3

Medidas amostrais dos estimadores dos parâmetros de regressão e precisão,

$$
\theta=\left(\beta_{0}, \beta_{1}, \beta_{2}, \beta_{3}, \beta_{4}, \beta_{5}, \phi\right)^{\top}=(1,0,0,1,1,20)^{\top} ; n=20 .
$$

\begin{tabular}{ccccccccccc}
\hline \hline Distr. & Est. & Média & DP & Viés & REQM & Assim. & Curt. & TCob. & LI & LS \\
\hline \multirow{5}{*}{$\mathcal{N}(0,1)$} & $\widehat{\beta_{0}}$ & 1,023 & 0,166 & 0,023 & 0,17 & 0,09 & 0,07 & 89,9 & 0,746 & 1,299 \\
& $\widehat{\beta_{1}}$ & $-0,001$ & 0,149 & $-0,001$ & 0,15 & 0,00 & $-0,05$ & 90,6 & $-0,245$ & 0,243 \\
& $\widehat{\beta_{2}}$ & 0,001 & 0,167 & 0,001 & 0,17 & $-0,00$ & 0,06 & 91,1 & $-0,273$ & 0,274 \\
& $\widehat{\beta_{3}}$ & 1,026 & 0,187 & 0,026 & 0,19 & 0,13 & 0,08 & 90,1 & 0,719 & 1,334 \\
& $\widehat{\beta_{4}}$ & 1,021 & 0,149 & 0,021 & 0,15 & 0,11 & 0,07 & 89,8 & 0,775 & 1,268 \\
& $\widehat{\phi}$ & 31,6 & 14,0 & 11,6 & 18,2 & 2,54 & 18,93 & 92,5 & 11,8 & 51,4 \\
\hline \hline \multirow{6}{*}{$t_{4}$} & $\widehat{\beta}_{0}$ & 1,018 & 0,131 & 0,018 & 0,13 & 0,09 & 0,00 & 89,8 & 0,802 & 1,235 \\
& $\widehat{\beta}_{1}$ & 0,001 & 0,147 & 0,001 & 0,15 & 0,06 & 0,01 & 90,7 & $-0,237$ & 0,239 \\
& $\widehat{\beta}_{2}$ & $-0,002$ & 0,184 & $-0,002$ & 0,18 & $-0,04$ & 0,12 & 92,4 & $-0,302$ & 0,298 \\
& $\widehat{\beta}_{3}$ & 1,021 & 0,149 & 0,021 & 0,15 & 0,15 & 0,20 & 90,5 & 0,780 & 1,262 \\
& $\widehat{\beta}_{4}$ & 1,022 & 0,234 & 0,022 & 0,24 & 0,13 & 0,06 & 90,0 & 0,642 & 1,402 \\
& $\widehat{\phi}$ & 31,1 & 13,2 & 11,1 & 17,2 & 1,90 & 6,87 & 92,8 & 11,9 & 50,3 \\
\hline \hline & $\widehat{\beta}_{0}$ & 1,016 & 0,141 & 0,016 & 0,14 & 0,07 & 0,16 & 90,6 & 0,781 & 1,251 \\
& $\widehat{\beta}_{1}$ & 0,000 & 0,087 & 0,000 & 0,09 & 0,01 & 0,05 & 91,7 & $-0,143$ & 0,143 \\
\multirow{2}{*}{$\operatorname{Exp}(2)$} & $\widehat{\beta}_{2}$ & $-0,002$ & 0,102 & $-0,002$ & 0,10 & 0,04 & $-0,01$ & 91,3 & $-0,170$ & 0,167 \\
& $\widehat{\beta}_{3}$ & 1,014 & 0,213 & 0,014 & 0,21 & 0,05 & $-0,04$ & 89,8 & 0,667 & 1,362 \\
& $\widehat{\beta}_{4}$ & 1,018 & 0,182 & 0,018 & 0,18 & 0,12 & 0,12 & 90,1 & 0,716 & 1,320 \\
& $\widehat{\phi}$ & 30,9 & 13,2 & 10,9 & 17,1 & 2,11 & 9,82 & 93,0 & 11,9 & 49,8 \\
\hline \hline
\end{tabular}

que os valores dos coeficiente de assimetria e curtose estão próximos de zero para os parâmetros de regressão e, acima de zero para o parâmetro de precisão, isto para as três distribuições analisadas. Uma diferença mais significativa nos resultados quando mudamos a matriz regressora é observada nas taxas de cobertura dos intervalos de confiança, que ficaram um pouco abaixo do valor esperado $95 \%$. Por exemplo, a taxa de cobertura para o parâmetro $\beta_{4}$ nas distribuições $\mathcal{N}(0,1), t_{4}$ e $\operatorname{Exp}(2)$ foi de $89,8 \%$, $90,0 \%$ e $90,1 \%$, respectivamente. Há de se ressaltar que estamos simulando um caso 
extremo, em que o tamanho da amostra e do paràmetro $\phi$ são pequenos. Em relação às estimativas intervalares, nota-se que os intervalos apresentam diferentes amplitudes médias. Por exemplo, a amplitude média do intervalo de confiança do parâmetro $\beta_{1}$ para as distribuições $\mathcal{N}(0,1), t_{4}$ e $\operatorname{Exp}(2)$ é $0,49,0,48$ e 0,29 unidades, respectivamente. Este fato pode ser explicado pelas diferenças nas formas das distribuiçōes utilizadas para gerar as covariadas consideradas na análise.

Por fim, percebemos que nesse modelo de regressão beta, em que a resposta média é modelada através de uma estrutura de regressão, adotando a função logito como função de ligação, os estimadores de máxima verossimilhança para os parâmetros de regressão apresentam boas propriedades em amostras finitas, pois mostraram-se quase não viciados e com uma distribuição próxima da distribuição normal de referência.

\subsection{Tamanho dos Testes}

Nesta seção apresentamos alguns resultados de simulação para avaliar os tamanhos dos testes. Comparamos os desempenhos de três testes, isto é, o da razão de verossimilhanças, escore e Wald. Os desempenhos são avaliados em função da proximidade das probabilidades de rejeição da hipótese nula, sendo esta verdadeira (probabilidade do erro tipo I), aos respectivos níveis nominais dos testes. Adicionalmente, realizamos uma análise da qualidade da aproximação das três estatísticas dos testes por uma distribuição qui-quadrado adequada.

Para todas as simulações, a hipótese nula considerada foi $\mathcal{H}_{0}: \beta_{1}=\beta_{2}=0$ e os níveis nominais adotados foram $10 \%, 5 \%, 1 \%$ e $0,5 \%$. O número de replicações de Monte Carlo foi novamente fixado em 10000.

Na. Tabela 4.4 apresentamos os resultados de simulação supondo que os valores 
Tabela 4.4

Tamanhos observados dos testes; 4 covariáveis uniformes;

$$
\text { Teste } \mathcal{H}_{0}: \beta_{1}=\beta_{2}=0 \times \mathcal{H}_{1}: \beta_{1} \neq 0 \text { ou } \beta_{2} \neq 0
$$

$\theta=\left(\beta_{0}, \beta_{1}, \beta_{2}, \beta_{3}, \beta_{4}, \phi\right)^{\top}=(1,0,0,1,1,120)^{\top}$.

\begin{tabular}{c|rcc|ccc|ccc}
\hline \multicolumn{9}{c}{$n=20$} & \multicolumn{3}{c}{$n=30$} & \multicolumn{3}{c}{$n=50$} \\
\hline \hline Nominal & RV & Escore & Wald & RV & Escore & Wald & RV & Escore & Wald \\
\hline $10 \%$ & 16,8 & 13,0 & 20,5 & 15,2 & 12,7 & 17,5 & 13,1 & 11,9 & 14,4 \\
$5 \%$ & 9,9 & 6,2 & 13,2 & 8,5 & 6,4 & 10,6 & 7,1 & 5,9 & 8,2 \\
$1 \%$ & 2,9 & 0,8 & 5,2 & 2,3 & 1,0 & 3,7 & 1,6 & 1,0 & 2,4 \\
$0,5 \%$ & 1,6 & 0,3 & 3,7 & 1,2 & 0,4 & 2,4 & 0,8 & 0,4 & 1,3 \\
\hline \hline
\end{tabular}

verdadeiros dos parâmetros são $\beta_{0}=1, \beta_{1}=0, \beta_{2}=0, \beta_{3}=1, \beta_{4}=1 \mathrm{e} \phi=120 \mathrm{e}$ matriz de covariadas geradas da distribuição $\mathcal{U}(0,1)$. Para cada tamanho amostral e cada nível nominal considerado, calculamos as taxas de rejeição de cada teste, isto é, estimamos, via simulação, $P\left(\omega_{1}>x_{\alpha}\right), P\left(\omega_{2}>x_{\alpha}\right)$ e $P\left(\omega_{3}>x_{\alpha}\right)$, em que $\omega_{1}, \omega_{2}$ e $\omega_{3}$ são como vistos nas Seções 3.6.1 a 3.6 .3 e $x_{\alpha}$ representa o quantil de ordem $(1-\alpha)$ da distribuição $\chi_{2}^{2}$.

Observa-se que os tamanhos empíricos dos testes aproximam-se dos níveis de significância nominais com o aumento do tamanho amostral, conforme esperado. Destaca-se ainda na Tabela 4.4, o fato de que os três testes são liberais, pois seus tamanhos empíricos estão sempre acima do nível nominal adotado. Verifica-se que o tamanho do teste escore é o que mais se aproxima do nível nominal. Por exemplo, para o nivel nominal de $5 \%$ e tamanho amostral $n=20$, seu valor é $6,2 \%$, enquanto que os valores correspondentes para os testes de RV e Wald são $9,9 \%$ e 13, 2\%, respectivamente. O tamanho do teste de Wald apresentou-se mais distante dos niveis nominais. Para $n=20$ e um nível nominal de $10 \%$, encontramos um valor de $20,5 \%$ para o tamanho do teste. 
Com o objetivo de avaliar a qualidade da aproximação das distribuições das três estatísticas por uma distribuição $\chi_{2}^{2}$, construímos gráficos de quantis ("q-q plot") para as estatísticas da razão de verossimilhanças, escore e Wald, para cada tamanho amostral analisado na Tabela 4.4. Os gráficos foram construídos com os valores ordenados de cada estatística contra quantis correspondentes da distribuição $\chi_{2}^{2}$. A Figura 4.1 ilustra os resultados obtidos. Se a distribuição da estatística analisada apresenta. uma boa aproximação pela distribuição $\chi_{2}^{2}$, espera-se que esse gráfico seja próximo de uma reta. Para facilitar essa avaliação, constrúmos uma reta tracejada considerada ideal, onde os pontos deveriam estar caso houvesse uma perfeita concordância entre os quantis amostrais da distribuição da estatística do teste analisado e os quantis correspondentes da distribuição de referência $\left(\chi_{2}^{2}\right)$. A análise da Figura 4.1 confirma os resultados encontrados para os tamanhos dos testes na Tabela 4.4. Verifica-se que os pontos do gráfico referentes à estatística escore se apresentam, de forma geral, mais próximo da reta tracejada do que os dos gráficos referentes às estatísticas de razão de verossimilhanças e de Wald, que estão sempre acima dessa reta. Esse fato explica os tamanhos grandes dos testes encontrados para essas duas estatísticas.

Verifica-se também na Figura 4.1, que a estatística de Wald tem quantis amostrais bem maiores que os correspondentes quantis da distribuição $\chi_{2}^{2}$. Este fato ocorre para todos os tamanhos amostrais considerado nas análises, sendo mais agravante para $n=20$. Também, podemos verificar que o efeito do tamanho da amostra $(n)$ está intimamente relacionado com a qualidade da aproximação das estatísticas do teste pela distribuição de referência $\left(\chi_{2}^{2}\right)$. Com o aumento de $n$, verifica-se uma melhor aproximação das três estatísticas pela distribuição $\chi_{2}^{\frac{2}{2}}$.

De forma análoga ao procedimento realizado para as estimativas dos parâmetros, realizamos as mesmas simulaçōes atribuindo os mesmos valores para os parâmetros de regressão, mas modificando o parâmetro $\phi$ de 120 para 20 unidades (menor precisão). 
$R V, n=20$

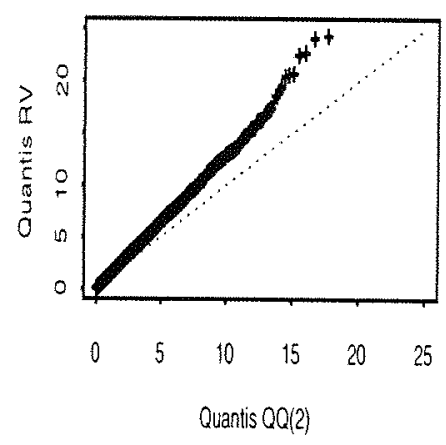

$R V, n=30$

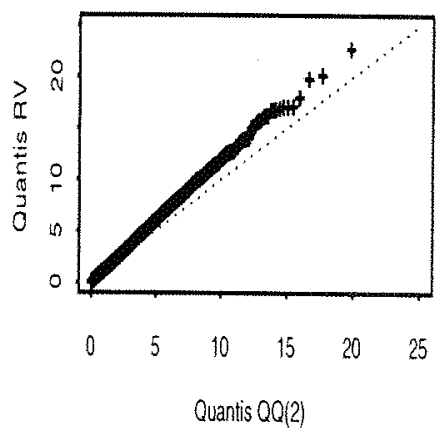

$R V, n=50$

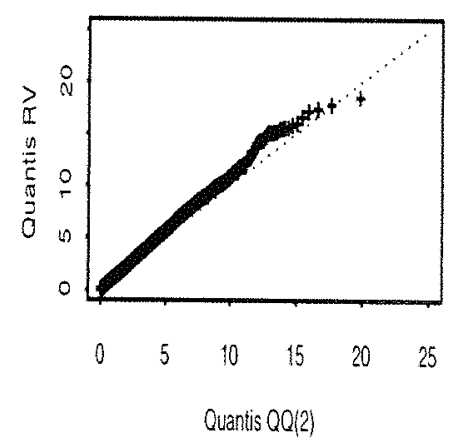

Escore, $n=20$

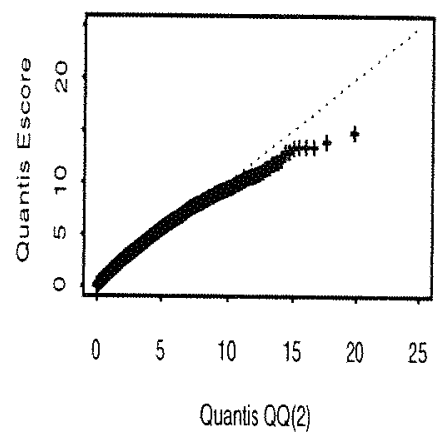

Escore, $n=30$

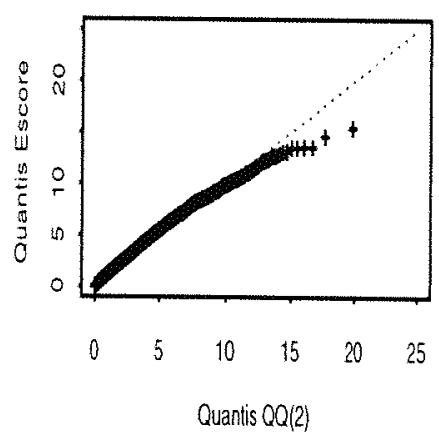

Escore, $n=50$

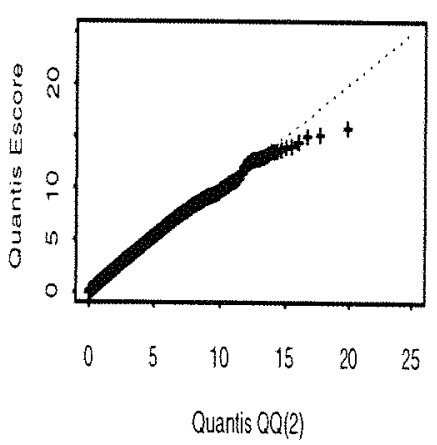

Wald, $n=20$

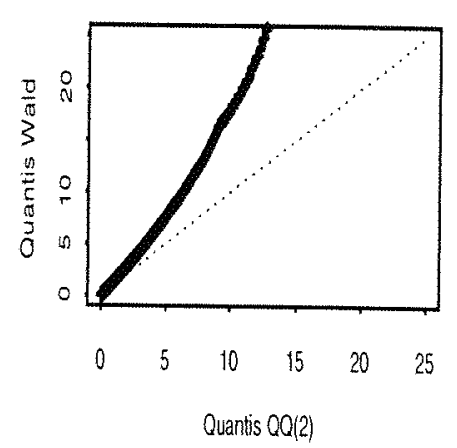

Wald, $n=30$

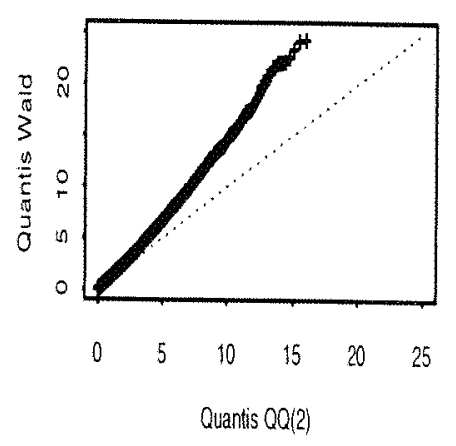

Wald, $n=50$

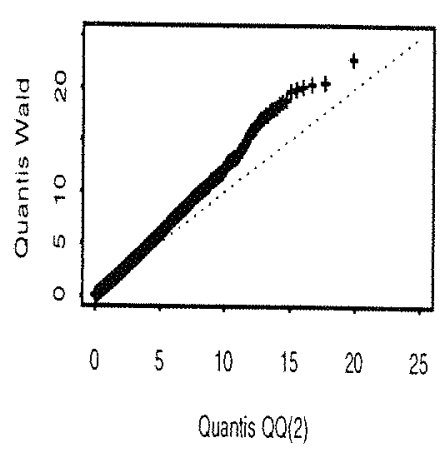

Figura 4.1: Gráficos Q-Q Plot para as estatísticas RV, Escore e Wald; $\theta=\left(\beta_{0}, \beta_{1}, \beta_{2}, \beta_{3}, \beta_{4}, \phi\right)^{\top}=(1,0,0,1,1,120)^{\top}$ 
Os resultados destas simulações para os tamanhos dos testes encontram-se na Tabela 4.5 .

\section{Tabela 4.5}

Tamanhos observados dos testes; 4 covariáveis uniformes

$$
\text { Teste } \mathcal{H}_{0}: \beta_{1}=\beta_{2}=0 \times \mathcal{H}_{1}: \beta_{1} \neq 0 \text { ou } \beta_{2} \neq 0
$$$$
\theta=\left(\beta_{0}, \beta_{1}, \beta_{2}, \beta_{3}, \beta_{4}, \phi\right)^{\top}=(1,0,0,1,1,20)^{\top} \text {. }
$$

\begin{tabular}{c|ccc|ccc|ccc}
\hline \multicolumn{4}{c}{$n=20$} & \multicolumn{3}{c}{$n=30$} & \multicolumn{3}{c}{$n=50$} \\
\hline \hline Nominal & RV & Escore & Wald & RV & Escore & Wald & RV & Escore & Wald \\
\hline $10 \%$ & 18,2 & 13,3 & 22,3 & 14,4 & 11,7 & 16,8 & 12,6 & 11,2 & 14,1 \\
$5 \%$ & 11,0 & 6,8 & 15,1 & 8,2 & 6,0 & 10,5 & 6,7 & 5,3 & 8,2 \\
$1 \%$ & 3,4 & 0,9 & 6,4 & 2,3 & 1,0 & 3,7 & 1,7 & 1,0 & 2,4 \\
$0,5 \%$ & 2,1 & 0,4 & 4,4 & 1,3 & 0,4 & 2,5 & 1,0 & 0,5 & 1,5 \\
\hline \hline
\end{tabular}

Observa-se que os tamanhos empíricos dos testes aproximam-se dos níveis de significância nominais com o aumento do tamanho amostral, conforme esperado. Notase ainda na Tabela 4.5 que houve um pequeno aumento no valor dos tamanhos dos testes para $n=20$ e, um ligeiro decréscimo para $n=30$ e $n=50$, comparados aos resultados obtidos na Tabela 4.4. Por exemplo, para $n=20$ e nível nominal de $10 \%$ os tamanhos dos testes de razão de verossimilhanças, escore e Wald passaram de $16,8 \%, 13,0 \%$ e $20,5 \%$ para $18,2 \%, 13,3 \%$ e $22,3 \%$, respectivamente, enquanto que para $n=30$ e nível nominal de $5 \%$ os tamanhos dos testes de razão de verossimilhanças, escore e Wald passaram de $8,5 \%, 6,4 \%$ e $10,6 \%$ para $8,2 \%, 6,0 \%$ e $10,5 \%$, respectivamente. Os testes apresentaram-se novamente liberais, sendo que o teste escore foi aquele que apresentou melhor desempenho em termos de tamanho.

A Figura 4.2 apresenta os gráficos de quantis ("q-q plot") para as estatísticas da razão de verossimilhanças, escore e Wald, para cada tamanho amostral analisado na Tabela 4.5. A análise da Figura 4.2 confirma os resultados encontrados para os 


$$
R V, n=20
$$

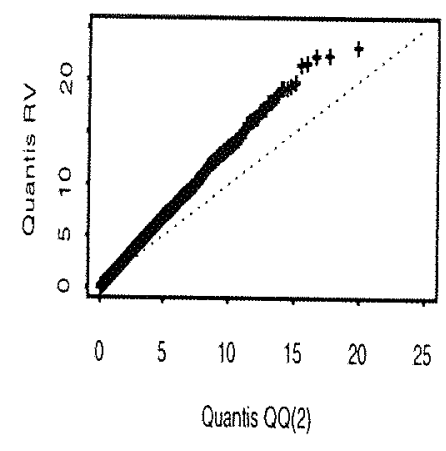

RV,$n=30$

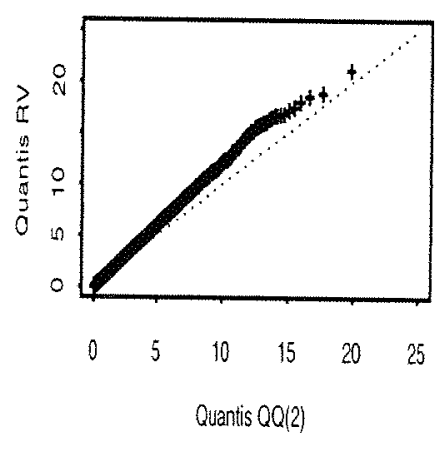

RV, $n=50$

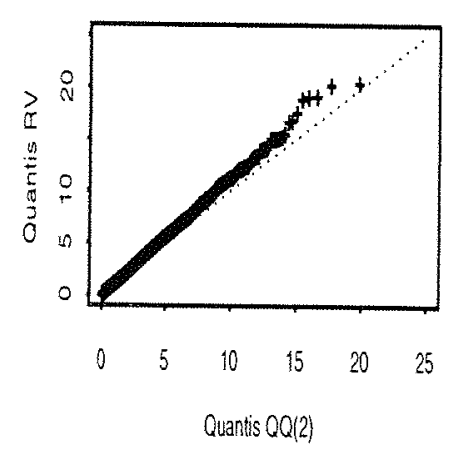

Escore, $n=20$

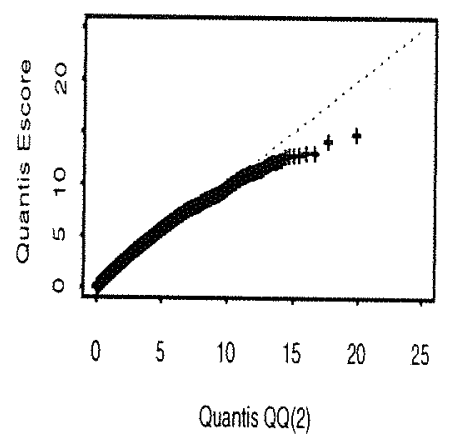

Escore, $n=30$

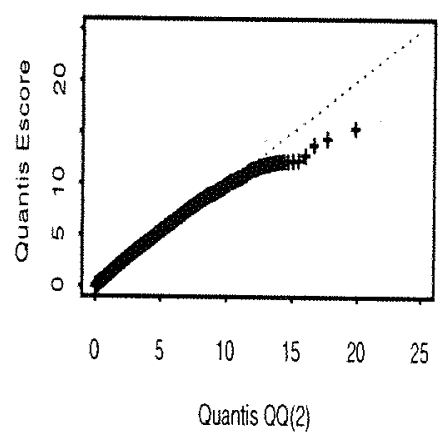

Escore, $n=50$

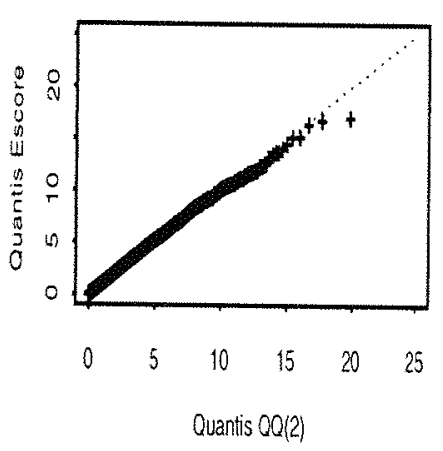

Wald, $n=20$

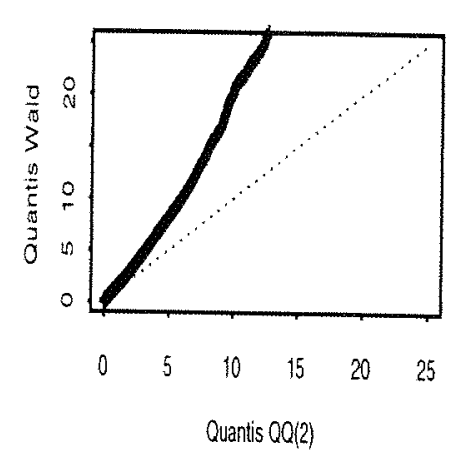

Wald, $n=30$

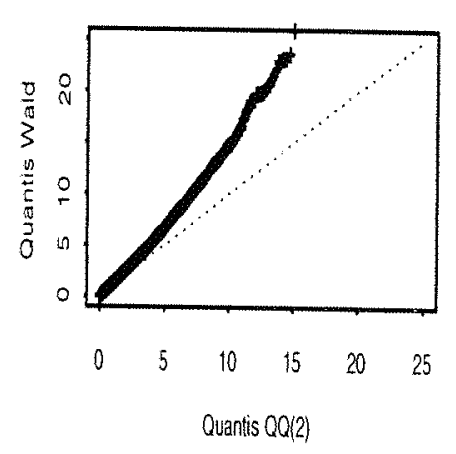

Wald, $n=50$

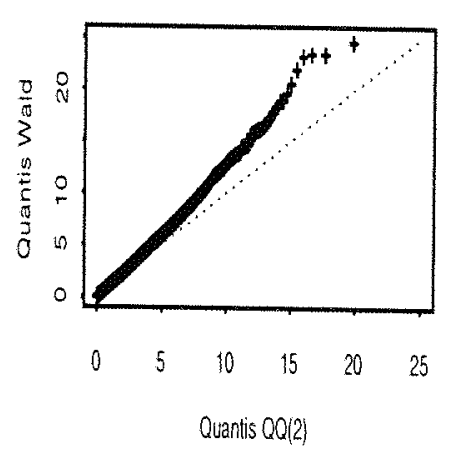

Figura 4.2: Gráficos Q-Q Plot para as estatisticas RV, Escore e Wald; $\theta=\left(\beta_{0}, \beta_{1}, \beta_{2}, \beta_{3}, \beta_{4}, \phi\right)^{\top}=(1,0,0,1,1,20)^{\top}$. 
tamanhos dos testes na Tabela 4.5. Como ocorrido na análise da Figura 4.1, verificase novamente que os pontos do gráfico referentes à estatística escore se apresentam, de forma geral, mais próximo da reta tracejada do que os dos gráficos referentes às estatísticas de razão de verossimilhanças e de Wald, que estão sempre acima dessa reta.

Dos comentários realizados nas Tabelas 4.4 e 4.5, podemos concluir que o valor do parâmetro de precisão não influencia significativamente nos tamanhos empíricos dos testes.

Finalmente, apresentamos na Tabela 4.6 os resultados de simulação para os tamanhos dos testes, quando a matriz de regressores $X$ é gerada das distribuições $\mathcal{N}(0,1)$, $t_{4}$ e $\operatorname{Exp}(2)$. Fixamos o tamanho amostral em $n=20$ e os valores verdadeiros para os parâmetros foram $\beta_{0}=1, \beta_{1}=0, \beta_{2}=0, \beta_{3}=1, \beta_{4}=1$ e $\phi=20$.

\section{Tabela 4.6}

Tamanhos observados dos testes; 4 covariáveis; $n=20$;

Teste $\mathcal{H}_{0}: \beta_{1}=\beta_{2}=0 \times \mathcal{H}_{1}: \beta_{1} \neq 0$ ou $\beta_{2} \neq 0$;

$$
\theta=\left(\beta_{0}, \beta_{1}, \beta_{2}, \beta_{3}, \beta_{4}, \phi\right)^{\top}=(1,0,0,1,1,20)^{\top} .
$$

\begin{tabular}{c|rrr|rrr|rrr}
\hline \multicolumn{3}{c}{$\mathcal{N}(0,1)$} & \multicolumn{4}{c}{$t_{4}$} & \multicolumn{3}{c}{$\operatorname{Exp}(2)$} \\
\hline \hline Nominal & RV & Escore & Wald & RV & Escore & Wald & RV & Escore & Wald \\
\hline $10 \%$ & 18,0 & 13,6 & 21,7 & 18,1 & 13,8 & 21,9 & 17,9 & 14,1 & 21,1 \\
$5 \%$ & 10,7 & 6,7 & 14,3 & 10,8 & 7,1 & 14,4 & 10,9 & 7,2 & 13,9 \\
$1 \%$ & 3,3 & 1,1 & 5,9 & 3,5 & 1,1 & 6,3 & 3,3 & 1,1 & 5,9 \\
$0,5 \%$ & 2,0 & 0,4 & 4,2 & 2,1 & 0,3 & 4,6 & 2,0 & 0,4 & 4,2 \\
\hline \hline
\end{tabular}

Observa-se na Tabela 4.6 que os tamanhos empíricos dos testes podem diferir ligeiramente dependendo da matriz de regressores $X$. Os tamanhos observados dos testes da razão de verossimilhanças, escore e Wald para a distribuição $\mathcal{N}(0,1)$ estão sempre mais próximos dos níveis nominais adotados em relação aos obtidos pela dis- 
tribuição $t_{4}$. O mesmo fato ocorre se compararmos a distribuição $\mathcal{N}(0,1)$ com a distribuição $\operatorname{Exp}(2)$, a menos do tamanho do teste de Wald, em que os valores observados na distribuição $\operatorname{Exp}(2)$ estão mais próximos do níveis nominais adotados com relação aos obtidos pela distribuição $\mathcal{N}(0,1)$. Por exemplo, para um nível nominal de $10 \%$, os valores encontrados para a estatística de Wald para as distribuições $\mathcal{N}(0,1)$ e $\operatorname{Exp}(2)$ foram $21,68 \%$ e $21,07 \%$, respectivamente.

Dentre os três testes analisados (RV, Escore e Wald), verifica-se que o tamanho empírico do teste escore é o que mais se aproxima dos níveis nominais, independente da distribuição adotada para as covariadas. Para o nível nominal de $0,5 \%$, o teste escore mostrou-se um pouco conservativo para as três distribuições consideradas. Os valores obtidos para o tamanho empírico deste teste para as distribuições $\mathcal{N}(0,1), t_{4}$ e $\operatorname{Exp}(2)$ foram $0,43 \%, 0,28 \%$ e $0,41 \%$, respectivamente.

A Figura 4.3 apresenta os gráficos de quantis ("q-q plot") para as estatísticas da razão de verossimilhanças, escore e Wald para cada distribuição considerada para as covariáveis analisado na Tabela 4.6. Como observado anteriormente, verifica-se que os pontos do gráfico referentes à estatística escore se apresentam, de forma geral, mais próximo da reta tracejada do que os dos gráficos referentes às estatísticas de razão de verossimilhanças e de Wald, que estão sempre acima dessa reta, isto para as três distribuições consideradas. Verifica-se também, que a estatística de Wald tem quantis amostrais bem maiores do que os correspondentes quantis da distribuição $\chi_{2}^{2}$, para as distribuições $\mathcal{N}(0,1), t_{4}$ e $\operatorname{Exp}(2)$ consideradas para as covariadas.

Finalmente, concluímos a partir das simulações, que o teste escore é o mais confiável no que diz respeito à probabilidade de erro de tipo I.

Realizamos uma simulação adicional utilizando a função de ligação complemento $\log -\log (g(\mu)=\log \{-\log (1-\mu)\})$. Os resultados foram semelhantes aos encontrados 
para a função de ligação logito. Devido a esse fato, decidimos omitir os resultados.

$R V, N(0,1)$

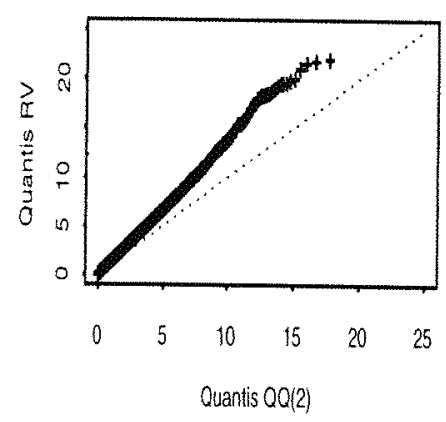

$R V, t(4)$

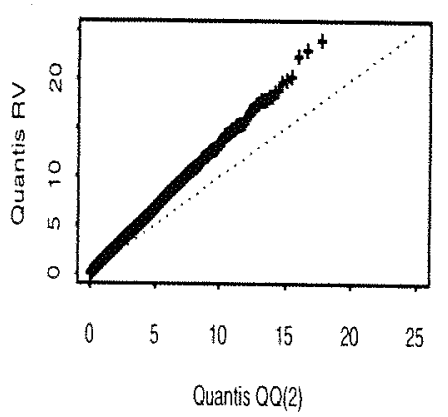

$R V, \operatorname{Exp}(2)$

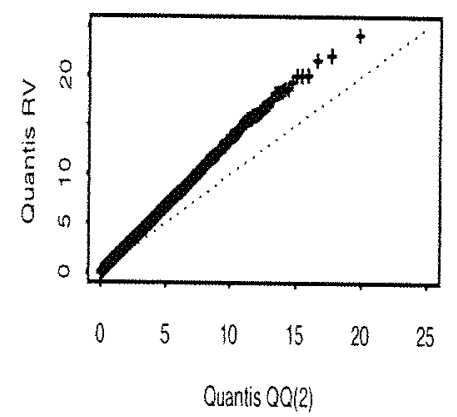

Escore, $N(0,1)$

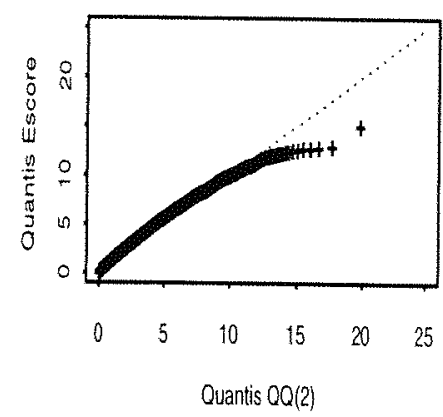

Escore, $t(4)$

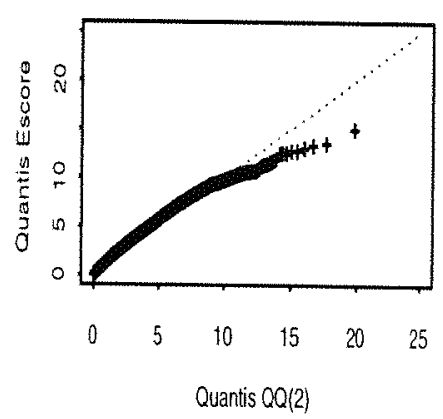

Escore, $\operatorname{Exp}(2)$

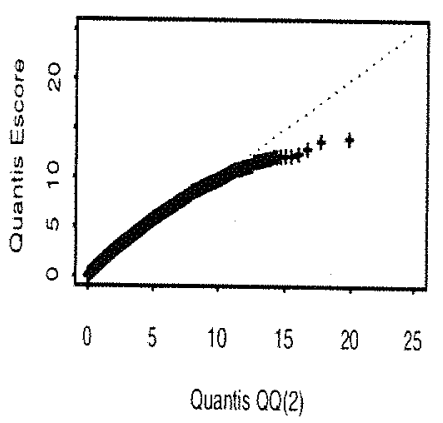

Wald , N(0,1)

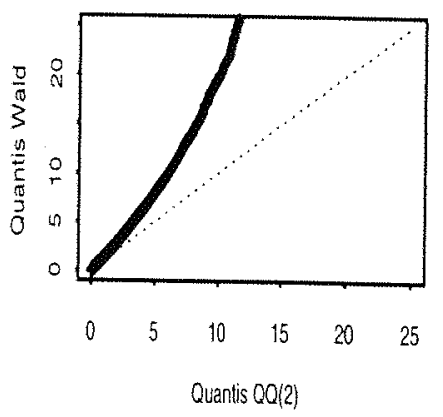

Wald , t(4)

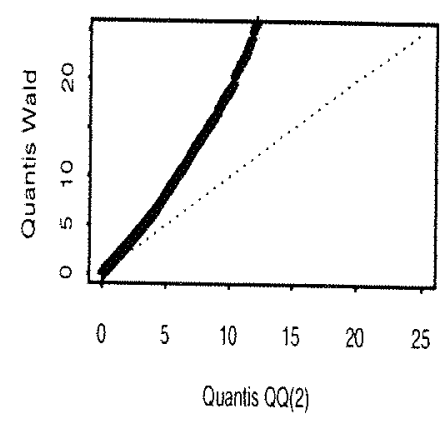

Wald, Exp(2)

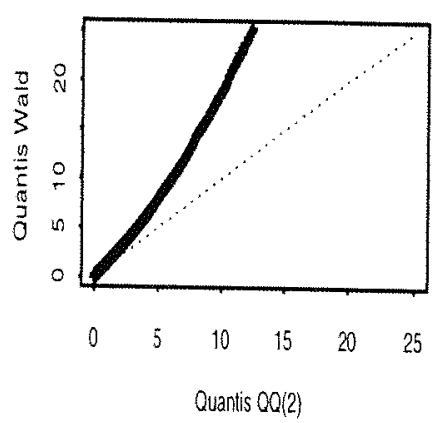

Figura 4.3: Gráficos Q-Q Plot para as estatísticas $R V$, Escore e Wald;

$$
\theta=\left(\beta_{0}, \beta_{1}, \beta_{2}, \beta_{3}, \beta_{4}, \phi\right)^{\top}=(1,0,0,1,1,20)^{\top} ; n=20 .
$$




\section{Aplicações}

Neste capítulo apresentamos duas aplicações do modelo de regressão beta a conjuntos de dados reais e uma aplicação utilizando dados simulados. Todos os cálculos computacionais foram realizados usando a linguagem de programação matricial $\mathrm{Ox}$ (Doornik, 2001). A estimação foi realizada usando o algoritmo quasi-Newton BFGS e a escolha dos valores iniciais para os parâmetros desconhecidos segue a sugestão de Ferrari e Cribari-Neto (2004) que foi descrita na Seção 3.5. O programa que foi utilizado para a análise do conjunto de dados de Oxidação de Amônia encontra-se no Apêndice $\mathrm{C}$.

\subsection{Oxidação de Amônia}

Considere inicialmente os dados de Oxidação' ${ }^{1}$ de Amônia descritos na Seção 2.4.1. O experimento ocorre da seguinte forma: em um reator que fica localizado dentro de uma planta industrial, o gás amônia $\left(\mathrm{NH}_{3}\right)$ reage com o oxigênio do ar $\left(\mathrm{O}_{2}\right)$ formando o óxido nítrico $(\mathrm{NO})$, que num processo contínuo e rápido, reage novamente com o

\footnotetext{
${ }^{1}$ Oxidação é uma reação química com o oxigênio.
} 
oxigênio do ar $\left(\mathrm{O}_{2}\right)$ formando o dióxido de nitrogênio $\left(\mathrm{NO}_{2}\right)$. Este, por sua vez, reage com a água $\left(\mathrm{H}_{2} \mathrm{O}\right)$ formando o ácido nítrico $\left(\mathrm{HNO}_{3}\right)$ e também o óxido nítrico (NO). O óxido nítrico (NO) produzido é, então, absorvido e reutilizado no processo de obtenção do ácido nítrico $\left(\mathrm{HNO}_{3}\right)$. Todas as reações químicas utilizadas nesse processo liberam muito calor, sendo necessário o resfriamento do sistema através da água que circula ao redor do reator.

Como visto anteriormente, a variável resposta $(y)$ corresponde à perda na conversão de amônia, que é a proporção de amônia que não foi convertida em ácido nítrico, sendo esta, uma medida inversa da eficiência total da planta industrial. $O$ interesse encontra-se na modelagem da proporção de amônia que não foi convertida em ácido nítrico pelas covariáveis "corrente de ar" $\left(x_{1}\right)$, "temperatura da água" $\left(x_{2}\right)$ (utilizada para o resfriamento do processo) e "concentração de ácido" $\left(x_{3}\right)$, medida como $10 \times($ concentração de ácido -50$)$.

A análise original deste conjunto de dados foi realizada por Brownlee (1965, p. 454), consistindo no ajuste de um modelo de regressão linear múltipla com as três variáveis explicativas, sem utilizar transformação na resposta, retirando posteriormente a variável concentração de ácido $\left(x_{3}\right)$ e repetindo a análise. Técnicas gráficas não foram usadas, como também não foi examinada a diferença residual entre as observações e o modelo ajustado. Essa análise foi estendida por Draper e Smith (1966, Exercício 6D), mas conservando a mesma estrutura. A análise mais detalhada de Daniel e Wood (1971, Capítulo 5) leva à retirada das observações 1,3,4 e 21. Cook (1979) pesquisa a presença de outliers múltiplos nesse conjunto de dados. Atkinson (1985, p. 129) propõe um modelo de regressão linear (homoscedástico) para $\log y$ com as covariadas $x_{1}, x_{2}, x_{1} x_{2}$ e $x_{1}^{2}$, o qual fornece $u m$ bom ajuste para todas as 21 observações, porém utiliza transformação logarítmica na resposta e adiciona dois termos de segunda ordem no preditor linear. Em suas análises, ele verifica que esse 
modelo fornece uma boa representação para os dados, mas ressalta que o mesmo é altamente dependente da observação 21 , que provoca a necessidade de inclusão de dois termos extra (quadráticos) na média da variável resposta transformada. Galea, Paula e Uribe-Opazo (2002) analisaram esse conjunto de dados adotando o seguinte modelo

$$
y_{i}=\beta_{0}+\beta_{1} x_{t 1}+\beta_{2} x_{t 2}+\beta_{3} x_{t 3}+\epsilon_{t}
$$

em que $\epsilon_{t}$ segue uma distribuição elíptica apropriada. Em particular, eles compararam os ajustes através de técnicas de influência local em modelos elípticos lineares, adotando para os erros as distribuições Normal, t-Student e exponencial potência. Para todos os modelos ajustados, os parâmetros $\beta_{0}, \beta_{1}$ e $\beta_{2}$ foram altamente significativos, enquanto que a hipótese de que $\beta_{3}=0$ não é rejeitada.

Nossa abordagem será diferente daquela utilizada nessas análises. Analisaremos esse conjunto de dados fazendo uso do modelo de regressão beta descrito no Capítulo 3. Para isso, assumiremos que a variável resposta $y_{t}$ (perda na conversão) tem uma distribuição beta com média $\mu_{t}$ e usando as demais variáveis como explicativas $\left(x_{1}, x_{2}\right.$ e $x_{3}$ ). Inicialmente, consideraremos o modelo

$$
g\left(\mu_{t}\right)=\beta_{0}+\beta_{1} x_{t 1}+\beta_{2} x_{t 2}+\beta_{3} x_{t 3},
$$

em que g representa a função de ligação logito (ver Seção 3.3).

Os resultados de estimação são dados na Tabela 5.1. Verifica-se que o nível descritivo marginal das variáveis explicativas "corrente de ar" e "temperatura da água" estão próximos de zero, indicando que essas variáveis são estatisticamente importantes para explicar a variável resposta "perda na conversão". No entanto, o nivel descritivo marginal da variável explicativa "concentração de ácido" é de 0,7386, o que indica que essa variável pode ser retirada do modelo, desde que sejam mantidas as outras duas. 
Tabela 5.1

Estimativas dos parâmetros do estudo de Oxidação de Amônia.

\begin{tabular}{ccccc}
\hline \hline Parâmetro & Estimativa & E. Padrão & E/E.Padrão & P-Valor \\
\hline$\beta_{0}$ & $-7,7298$ & 0,6789 & $-11,3866$ & 0,0000 \\
$\beta_{1}$ & 0,0294 & 0,0063 & 4,6894 & 0,0000 \\
$\beta_{2}$ & 0,0741 & 0,0188 & 3,9357 & 0,0001 \\
$\beta_{3}$ & 0,0029 & 0,0086 & 0,3337 & 0,7386 \\
$\phi$ & 2365,9 & 731,7 & & \\
\hline \hline
\end{tabular}

Nosso próximo passo é ajustar o modelo sem a variável "concentração de ácido" $\left(x_{3}\right)$. As estimativas de máxima verossimilhança desse novo modelo são dados na Tabela 5.2 .

Tabela 5.2

Estimativas dos parâmetros do estudo de Oxidação de Amônia sem considerar a variável "concentração de ácido".

\begin{tabular}{ccccc}
\hline Parâmetro & Estimativa & E. Padrão & E/E.Padrāo & P-Valor \\
\hline$\beta_{0}$ & $-7,5176$ & 0,2373 & $-31,6750$ & 0,0000 \\
$\beta_{1}$ & 0,0299 & 0,0061 & 4,8643 & 0,0000 \\
$\beta_{2}$ & 0,0744 & 0,0189 & 3,9356 & 0,0001 \\
$\phi$ & 2351,3 & 727,2 & & \\
\hline
\end{tabular}

Observa-se que todos os níveis descritivos marginais estão próximos de zero, indicando que as duas variáveis explicativas são estatisticamente significantes para o modelo. O pseudo $R_{p}^{2}$ da regressão estimada vale 0,9012 , enquanto que as medidas $R_{*}^{2}$ e $R_{L R}^{2}$, discutidos na Seção 3.7 , são 0,9150 e 0,9063 , respectivamente. Observase que as três medidas de qualidade do ajuste têm valores acima de $90 \%$ e estão relativamente próximas, indicando um bom ajuste do modelo.

Nota-se que $\exp \left(-1 \times \widehat{\beta_{1}}\right)=\exp (-0,0299) \simeq 0,97$, o que significa que se estima 
que, com a redução de uma unidade na variável explicativa "corrente de ar", a chance de perda na conversão diminui em $3 \%$, mantendo a temperatura da água fixa, ou seja, a redução do valor da "corrente de ar" corresponde a uma maior conversão da amônia em ácido nítrico. O mesmo fato ocorre para a variável explicativa "temperatura da água". Neste caso, $\exp \left(-1 \times \widehat{\beta}_{2}\right)=\exp (-0,0744) \simeq 0,93$, o que significa que sob uma redução de uma unidade na temperatura da água, que é utilizada para o resfriamento da reação, a chance de perda na conversão diminui em $7 \%$, considerando a corrente do ar inalterada. Adicionalmente, utilizando a expressão definida em (3.20), temos que um intervalo de confiança de aproximadamente $95 \%$ para a razão de chances $\exp \left(-1 \times \beta_{1}\right)$ é dado por $[0,959 ; 0,982]$. De forma análoga, um intervalo de confiança de aproximadamente $95 \%$ para a razão de chances $\exp \left(-1 \times \beta_{2}\right)$ é dado por $[0,895 ; 0,963]$. Agora, para calcularmos um intervalo de confiança para a média da variável resposta $\mu$ (perda média na conversão), utilizaremos a expressão definida em (3.21). Note que é necessário atribuir valores para as variáveis explicativas para a obtenção desse intervalo. Considerando que a "corrente de ar" e a "temperatura da água" são de 50 e 20 unidades, respectivamente, temos que o intervalo de confiança. de aproximadamente 95\% para a média da resposta $\mu$ (perda média na conversão) é dado por $[0,0093 ; 0,0121]$.

Alguns gráficos de diagnóstico são dados na Figura 5.1. Na Figura 5.1 (a) é apresentado o gráfico dos resíduos padronizados contra os índices das observações, enquanto que na Figura 5.1 (b) temos o gráfico dos resíduos componentes do desvio versus os índices das observações. O gráfico meio-normal dos resíduos componentes dos desvio com um envelope simulado é apresentado na Figura 5.1 (c), enquanto que na Figura 5.1 (d) é apresentado o gráfico dos resíduos padronizados versus $\widehat{\eta}_{t}$ (preditor linear). Finalmente, na Figura 5.1 (e) temos o gráfico dos $C_{t}$ contra $t$ e na Figura 5.1 (f) o gráfico dos elementos da diagonal de $\operatorname{GL}(\widehat{\beta}, \widehat{\phi})$ contra $\widehat{\mu}_{t}$. 

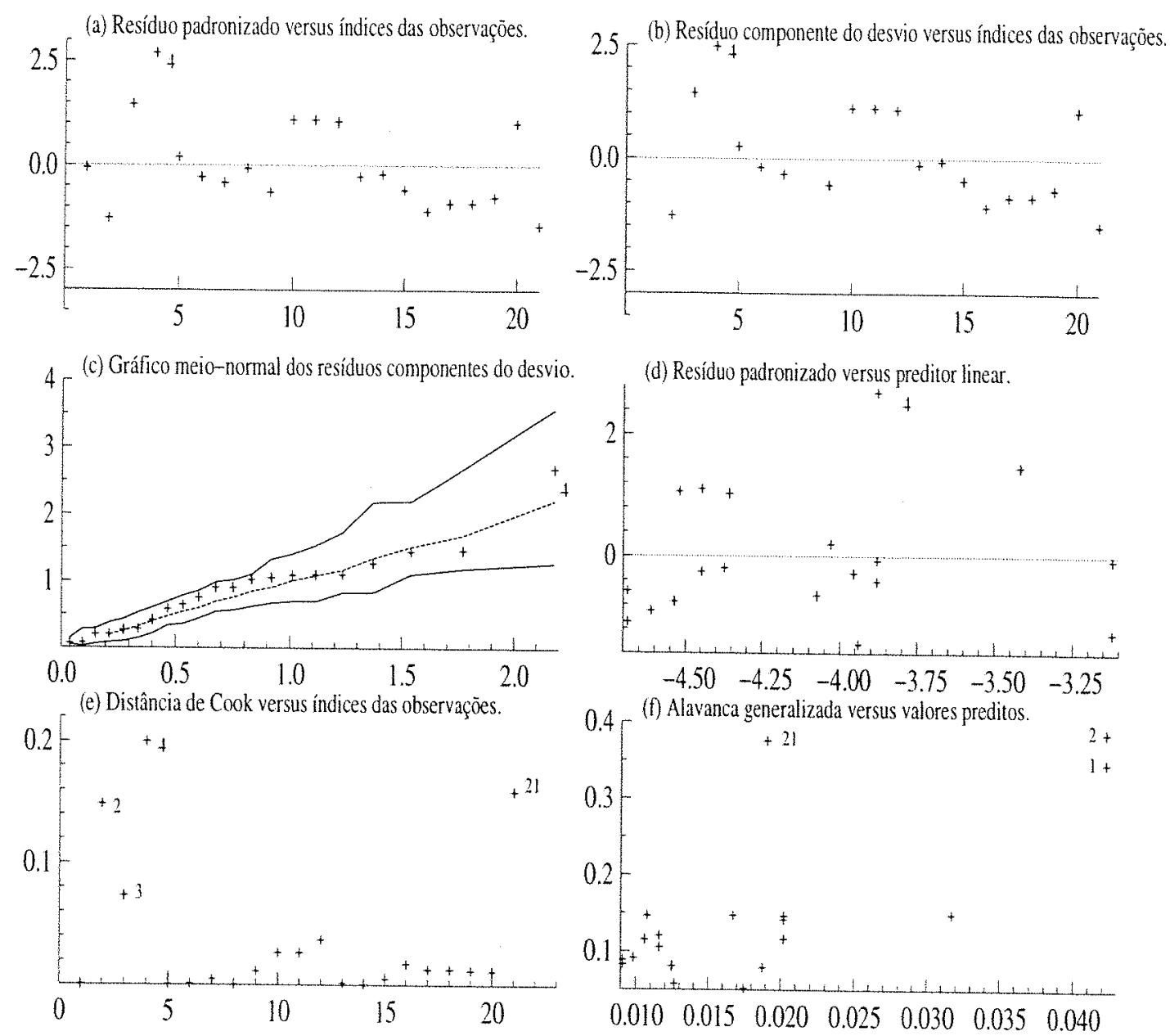

Figura 5.1: Gráficos de diagnóstico para os dados de Oxidação de Amônia.

Uma inspeção na Figura 5.1 revela que o maior resíduo tanto padronizado quanto o componente do desvio, em valor absoluto, corresponde à observação 4 . Nota-se na Figura 5.1 (c) que todos os pontos se encontram dentro do envelope simulado. $\mathrm{Na}$ Figura 5.1 (d), verifica-se que os pontos não apresentam nenhuma tendência. Em relação à distância de Cook, temos que $C_{4}$ é a que apresenta maior valor, seguido por $C_{21}$ e $C_{2}$. Em menor destaque, aparece a observação 3. Logo, a distância de Cook indica que as observações 4, 21 e 2 são as mais influentes. No gráfico de alavanca generalizada, nota-se que as observações 1, 2 e 21 estão fora do padrão, sendo que a 
observação 2 apresenta a maior alavanca generalizada.

Como os pontos 4 e 21 mais se destacaram nos gráficos de diagnóstico na Figura 5.1, resolvemos fazer uma análise da variação percentual das estimativas dos parâmetros quando retiramos esses pontos. A Tabela 5.3 apresenta os resultados obtidos desta análise. Nota-se que as estimativas pontuais dos $\beta$ 's não foram significativamente alteradas. A eliminação da observação 4 aumenta o efeito da variável "corrente de ar" e reduz o efeito da variável "temperatura da água", no entanto, elas continuaram significativas. O mesmo fato ocorre quando eliminamos a observação 21. Em geral, a eliminação das observações discrepantes não muda as conclusões inferenciais. Em relação ao parâmetro de precisão, a eliminação da observação 4 faz com que sua estimativa aumente em 43,46\%, enquanto que com a eliminação da observação 21 , sua estimativa aumenta apenas $13,01 \%$.

Tabela 5.3

Variação percentual das estimativas dos parâmetros retirando as observações discrepantes.

\begin{tabular}{cccccc}
\hline \hline Parâmetro & Estimativa & Sem 4 & V. Percentual & Sem 21 & V. Percentual \\
\hline$\beta_{0}$ & $-7,5176$ & $-7,5170$ & $-0,01 \%$ & $-7,4482$ & $-0,92 \%$ \\
$\beta_{1}$ & 0,0299 & 0,0344 & $15,05 \%$ & 0,0368 & $23,08 \%$ \\
$\beta_{2}$ & 0,0744 & 0,0602 & $-19,09 \%$ & 0,0519 & $-30,24 \%$ \\
$\phi$ & 2351,3 & 3373,2 & $43,46 \%$ & 2657,3 & $13,01 \%$ \\
\hline \hline
\end{tabular}

\subsection{Contaminação de Amendoim}

Nessa segunda aplicação, utilizamos os dados fornecidos em Draper e Smith (1998, p. 101-102). Como notado na Seção 2.4.2, os dados consistem de 34 lotes de 120 libras (aproximadamente $54 \mathrm{~kg}$ ) de amendoins. Para cada lote é fornecido o nível 
(médio) de aflatoxina $(x)$, dados em partes por bilhão (ppb) e a proporção de amendoins não contaminados no lote $(y)$. As aflatoxinas compõem-se de quatro substâncias principais identificadas como $\mathrm{B}_{1}, \mathrm{~B}_{2}, \mathrm{G}_{1}$ e $\mathrm{G}_{2}$. Elas são produzidas, principalmente, por dois fungos (bolores) denominados Aspergillus flavus e Aspergillus parasiticus, que se desenvolvem sobre muitos produtos agrícolas e alimentos, quando as condições de umidade do produto, umidade relativa do ar e temperatura ambiente são favoráveis. A ocorrência das aflatoxinas é maior no amendoim porque é o produto preferido pelo fungo e também porque, muitas vezes, há demora e chuvas no período de secagem após a colheita. Entretanto, sua maior incidência se dá quando o amendoim é batido, ensacado e armazenado com umidade elevada e quando reumedece depois de estar seco. Além do amendoim, a aflatoxina pode ser encontrada em muitos outros produtos, tais como milho e outros cereais, nozes etc. O efeito que ela pode causar nos seres humanos depende da dose e da freqüência com que é ingerida e pode ser agudo (letal ou não) ou subagudo (ver Fonseca, 2004). A Revista Ciência Hoje (2004, vol. 34) exibe uma matéria sobre a ocorrência de alto teor de aflatoxinas no produto brasileiro, relatando que esse fato reduz preços e afeta as exportaçōes. Segundo a reportagem, a Agência Nacional de Vigilância Sanitária (Anvisa) determinava, até 2001, que o limite máximo permitido, em alimentos destinados ao consumo humano, não deveria ultrapassar $30 \mathrm{ppb}$, somadas as aflatoxinas $B_{1}$ e $G_{1}$. Mas em 2003, uma legislação mais rígida entrou em vigor ( $R D C n^{\circ} 274$ da Anvisa), de acordo com as normas do Mercosul, criando novos parâmetros de avaliação: agora o somatório das aflatoxinas $\mathrm{B}_{1}, \mathrm{~B}_{2}, \mathrm{G}_{1}$ e $\mathrm{G}_{2}$ não deve superar $20 \mathrm{ppb}$, internalizando os limites estabelecidos no Mercosul (ver Barifouse, 2004).

Nosso objetivo é comparar os resultados dos ajustes das regressōes normal linear e beta. Posteriormente, vamos simular os valores da variável proporção de amendoins não contaminados no lote $(y)$ através da distribuição beta, a partir da variável 
explicativa nível de aflatoxina $(x)$. Nesse caso, o objetivo é ilustrar o fato de que a regressão linear pode produzir valores ajustados para a variável resposta fora do intervalo unitário.

Inicialmente, ajustamos um modelo de regressão normal linear simples, considerando $y$ como a proporção de amendoins não contaminados e $x$, o nível de aflatoxina. O modelo fica dado por

$$
y_{t}=\beta_{0}+\beta_{1} x_{t}+\epsilon_{t} \quad t=1, \ldots, 34
$$

com a suposição de que $\epsilon_{t} \sim N\left(0, \sigma^{2}\right)$, sendo os erros mutuamente independentes. Os resultados da estimação dos parâmetros estão apresentados na Tabela 5.4.

\section{Tabela 5.4}

Estimativas dos parâmetros do estudo de Contaminação de Amendoim usando o modelo de regressäo normal linear.

\begin{tabular}{ccccc}
\hline Parâmetro & Estimativa & E. Padrão & E/E.Padrão & P-Valor \\
\hline$\beta_{0}$ & 1,000020 & 0,00011000 & 9184,91 & 0,0000 \\
$\beta_{1}$ & $-0,000029$ & 0,00000234 & $-12,43$ & 0,0000 \\
\hline
\end{tabular}

Observa-se que a variável "nível de aflatoxina" é significante para explicar a variável "proporção de amendoins não contaminados". O fato de a estimativa do parâmetro $\beta_{1}$ ser negativa, indica que com o aumento do nível de aflatoxina no lote; há uma diminuição na proporção de amendoins não contaminados. O coeficiente de determinação da regressão é $R^{2}=82,9 \%$. Para avaliar as suposições do modelo, apresentamos alguns gráficos de diagnóstico (resíduo padronizado versus índices das observações, resíduo studentizado versus índices das observações, gráfico meio-normal dos resíduos studentizados, resíduo padronizado versus valores preditos, distância de Cook versus índices das observações e alavanca versus valores preditos) na Figura 5.2 . 

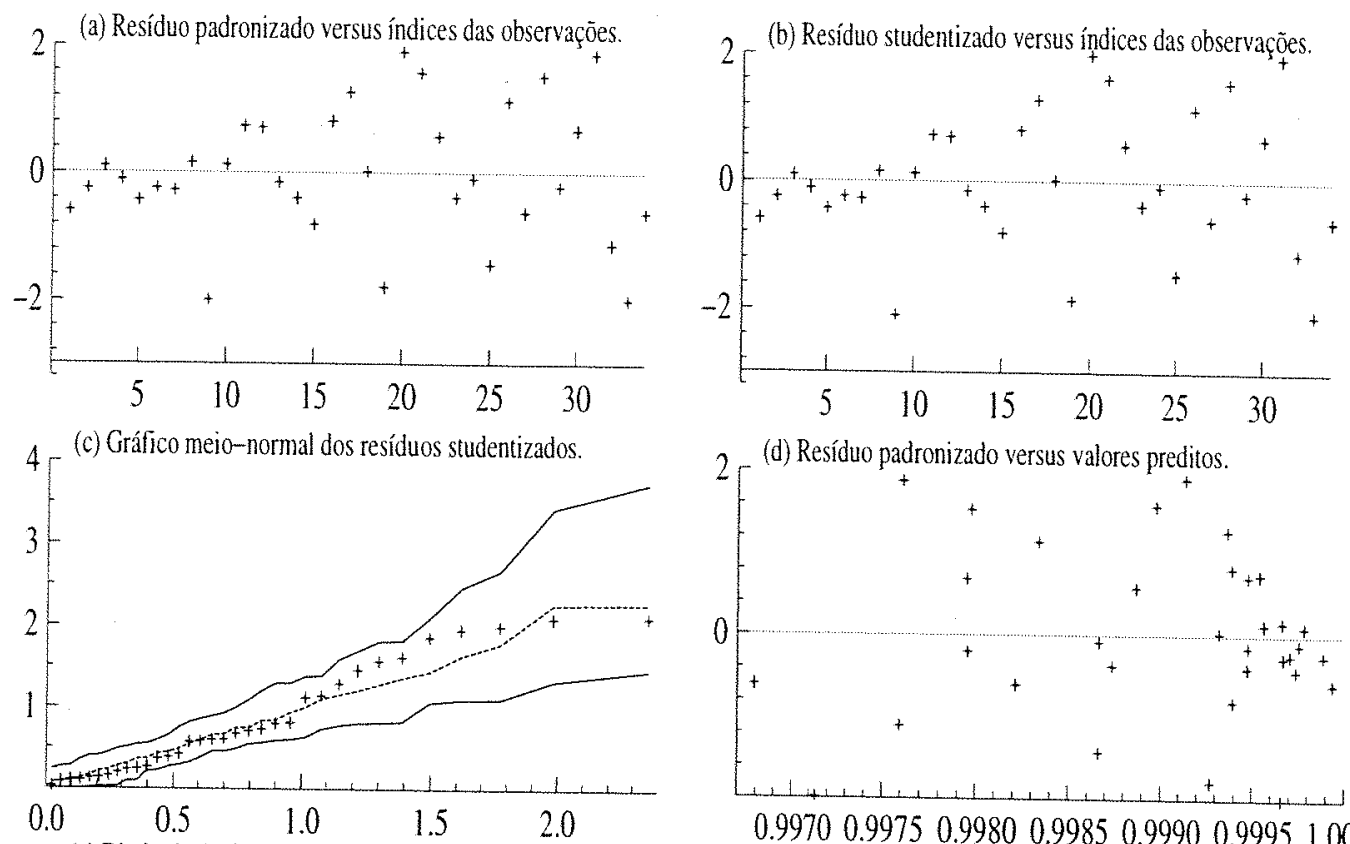

0.99700 .99750 .99800 .99850 .99900 .99951 .0000
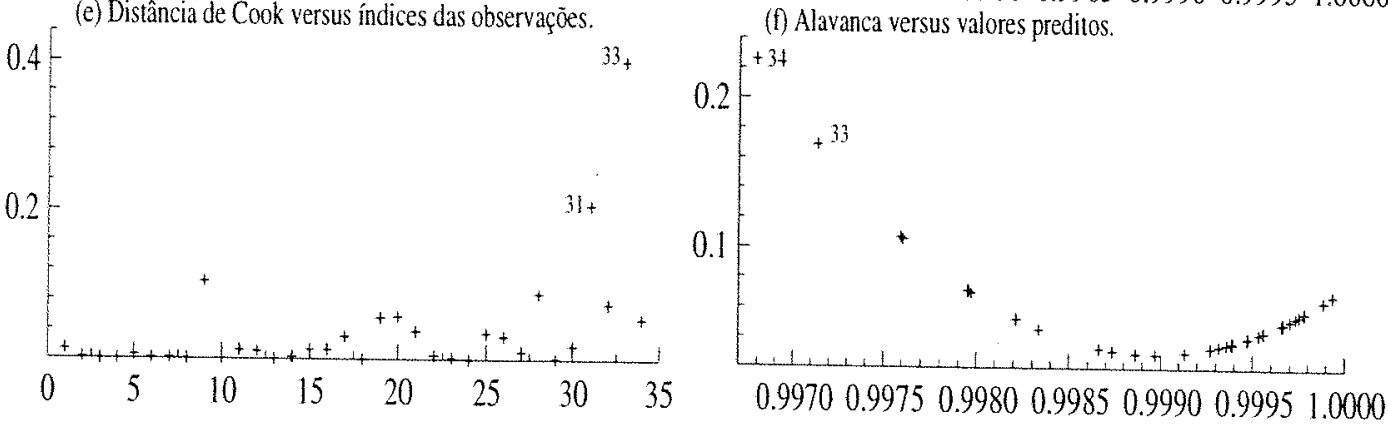

Figura 5.2: Gráficos de diagnóstico para os dados de Contaminação de Amendoim com o ajuste da regressão normal linear.

A Figura 5.2 não revela nenhum ponto de destaque nos gráficos dos resíduos padronizado e studentizado. Nota-se que todos os pontos encontram-se dentro do envelope simulado indicando que não há evidências de que o modelo forneceu mau ajuste. Em relação à distância de Cook, temos que a observação 33 é a que apresenta maior valor, seguido pela observação 31, sendo essas observações consideradas as mais influentes. No gráfico de alavanca, nota-se que as observações 34 e 33 estão fora do padrão, sendo as duas que correspodem aos maiores valores da variável explicativa. 
Realizamos agora, o ajuste do modelo de regressão beta para esses dados. Para isso, assumimos que a variável resposta $\left(y_{t}\right)$ tem uma distribuição beta com média $\mu_{t}$. Utilizando a variável explicativa $x$, consideramos o seguinte modelo

$$
g\left(\mu_{t}\right)=\beta_{0}+\beta_{1} x_{t} \quad t=1, \ldots, 34
$$

Os resultados da estimação usando a função de ligação logito são dados na Tabela 5.5 .

\section{Tabela 5.5}

Estimativas dos parâmetros do estudo de Contaminação de Amendoim usando o modelo de regressão beta.

\begin{tabular}{ccccc}
\hline \hline Parâmetro & Estimativa & E. Padrão & E/E.Padrão & P-Valor \\
\hline$\beta_{0}$ & 7,9459 & 0,1433 & 55,4440 & 0,0000 \\
$\beta_{1}$ & $-0,0226$ & 0,0021 & $-10,9826$ & 0,0000 \\
$\phi$ & 5424,1 & 1348,6 & & \\
\hline
\end{tabular}

Da Tabela 5.5, verifica-se que o nível descritivo marginal da variável explicativa "nível de aflatoxina" está próximo de zero, indicando que essa variável é estatisticamente importante para explicar a variável "proporção de amendoins não contaminados". Como ocorrido no ajuste da regressão normal linear, o valor da estimativa do parâmetro $\beta_{1}$ é negativo, indicando que com o aumento do nível de aflatoxina no lote, há uma diminuição da proporção de amendoins não contaminados. As medidas de qualidade do ajuste apresentaram os valores $R^{2}=0,6648, R_{*}^{2}=0,8148$ e $R_{L R}^{2}=0,7372$. A medida $R_{*}^{2}$ mostrou-se mais próxima do coeficiente de determinação do ajuste do modelo de regressão normal linear. Esse fato provavelmente ocorreu porque esta medida é obtida de forma semelhante a esse coeficiente.

Aqui $\exp \left(\widehat{\beta_{1}}\right)=\exp (-0,0226) \simeq 0,978$, o que significa que se estima que, com o aumento de uma unidade no "nível de aflatoxina", a chance da proporção de amen- 
doins não contaminados diminua em $2,2 \%$, ou seja, o aumento do valor do "nível de aflatoxina" corresponde a um aumento da proporção média de amendoins contaminados. Através da expressão (3.20), temos que um intervalo de confiança de aproximadamente $95 \%$ para a razão de chances $\exp \left(\beta_{1}\right)$ é dado por $[0,974 ; 0,982]$. De (3.21), considerando um "nível de aflatoxina" de $20 \mathrm{ppb}$ (valor máximo considerado para o consumo humano), temos que o intervalo de confiança de aproximadamente $95 \%$ para a média da resposta $\mu$ (proporção média de amendoins não contaminados) é dado por [0,99931;0,99955]. Analogamente, considerando um "nível de aflatoxina" de $80 \mathrm{ppb}$, temos que o intervalo de confiança de aproximadamente $95 \%$ para a média da resposta $\mu$ é dado por $[0,99746 ; 0,99816]$.

Na Figura 5.3 apresentamos os gráficos de diagnóstico do ajuste do modelo beta. Uma inspeção nessa figura revela que as observações 33 e 34 continuam sendo pontos alavanca. Uma diferença mais significativa está no gráfico das distâncias de Cook, onde destacou-se apenas a observação 34. A observação 31 não é mais destacada. Os demais gráficos apresentaram padrões semelhantes ao ajuste do modelo de regressão normal linear.

A Figura 5.4 apresenta o gráfico dos valores observados da variável "proporção de amendoins não contaminados" contra o "nível de aflatoxina", juntamente com as linhas correspondentes aos dois modelos ajustados. Verifica-se nesse gráfico o comportamento dos valores ajustados das duas regressões. Na regressão linear, obtém-se uma reta de pontos ajustados, enquanto que na regressão beta, temos uma curva. Nota-se também, que a regressão linear normal não forneceu valores ajustados fora do intervalo unitário.

Nesse conjunto de dados os valores observados de $y$ são próximos de 1 . Em situações como esta, é possível que o modelo de regressão linear normal forneça valores 

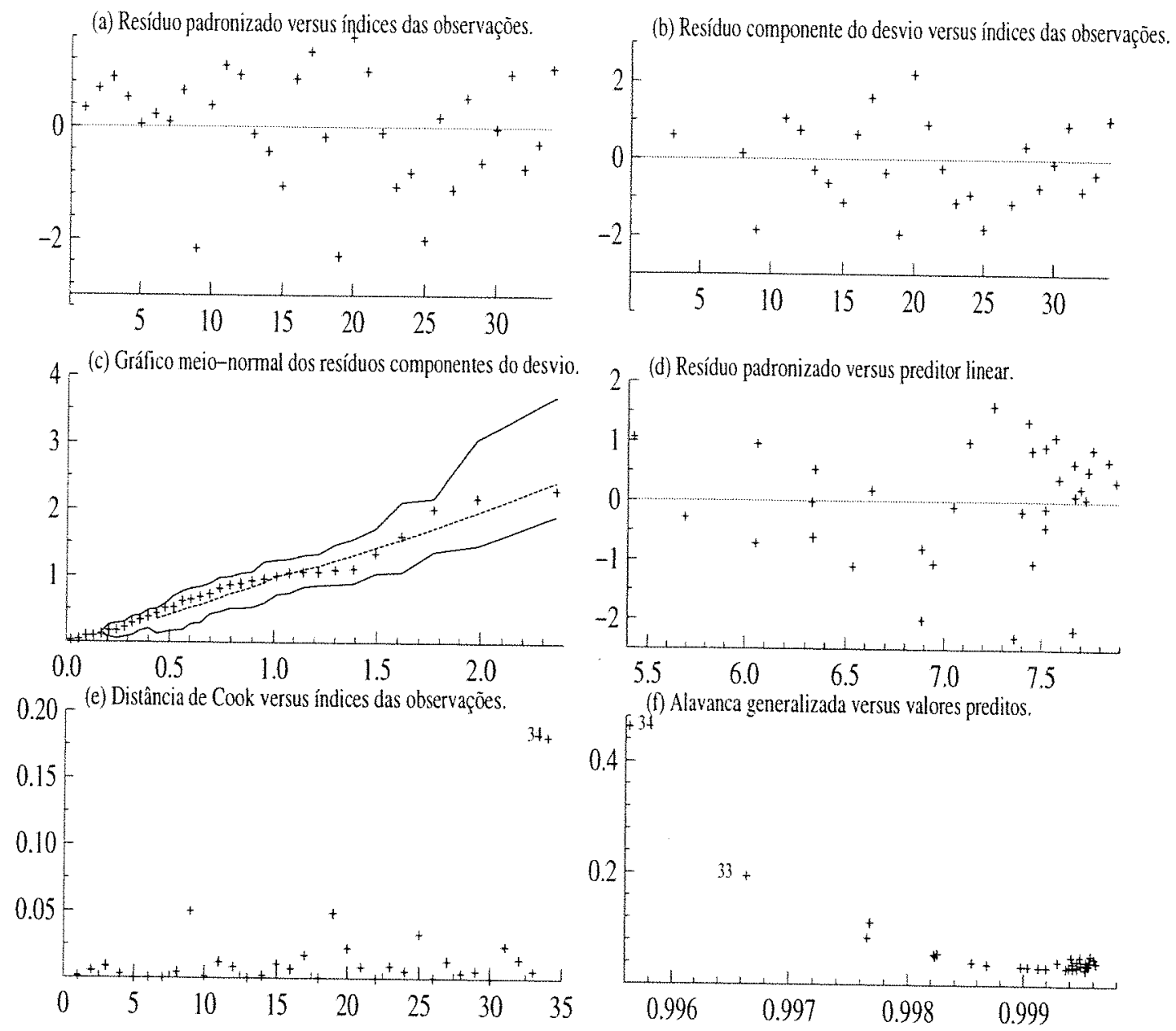

Figura 5.3: Gráficos de diagnóstico para os dados de contaminação de amendoim com o ajuste da regressão beta.

ajustados superiores a 1 , ou seja, estimativas fora do intervalo de valores possíveis para a resposta. Isto não ocorreu para os dados em questão, mas poderia ocorrer para outros conjuntos de dados. Para ilustrar uma situação como essa, utilizamos uma amostra obtida por simulação.

Os passos para a simulação foram: consideramos a mesma variável explicativa $x$ do conjunto de dados de Draper e Smith (1998, p. 101-102); atribuímos os seguintes 


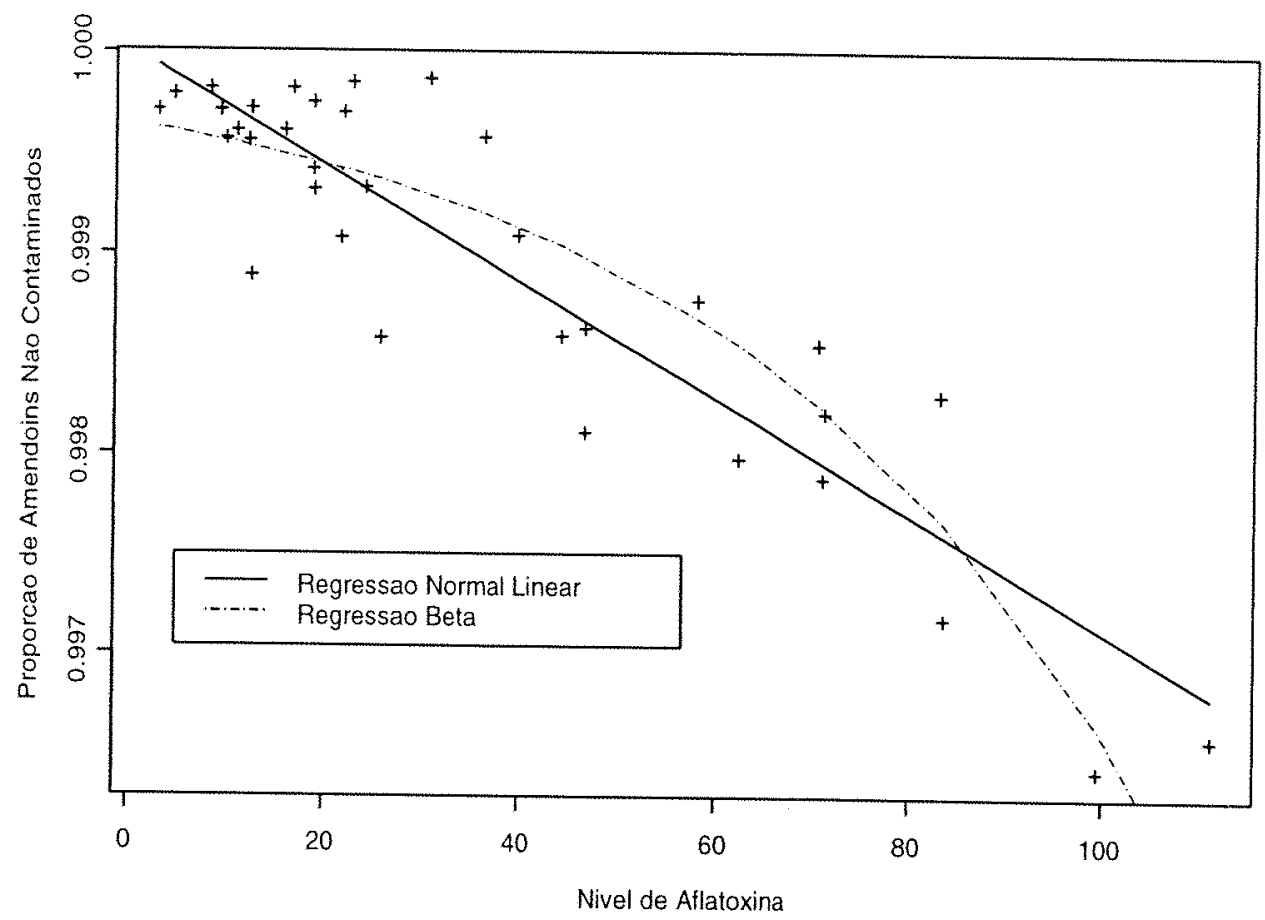

Figura 5.4: Gráficos dos ajustes das regressões normal linear e beta com ligação logito, para os dados de Contaminação de Amendoim.

valores como verdadeiros para os parâmetros $\beta_{0}=2, \beta_{1}=0,06$ e $\phi=2000$. Geramos 34 novos valores da distribuição beta com parâmetros $\left(\mu_{t} \phi,\left(1-\mu_{t}\right) \phi\right)$, sendo que os $\mu_{t}$ 's foram obtidos usando a função de ligação logito.

Na Tabela 5.6 apresentamos os valores simulados e preditos para a variável resposta, utilizando os ajustes dos modelos de regressão normal linear e beta com função de ligação logito.

Verifica-se na Tabela 5.6 que as observações 31 a 34 têm seus valores preditos obtidos do modelo de regressão normal linear acima de 1. Este fato não ocorre no ajuste do modelo de regressão beta, devido à sua construção teórica. Esta situação 
Tabela 5.6

Valores observados e preditos para o estudo de simulação usando os modelos de regressão normal linear e beta com função de ligação logito.

\begin{tabular}{cccccccc}
\hline \hline \multirow{2}{*}{ Obs. } & $y$ & \multicolumn{2}{c}{ Valores Preditos } & Obs. & $y$ & \multicolumn{2}{c}{ Valores Preditos } \\
\cline { 7 - 8 } & & Reg. Linear & Reg. Beta & & & Reg. Linear & Reg. Beta \\
\hline 1 & 0,89432 & 0,93749 & 0,89628 & 18 & 0,97259 & 0,95573 & 0,96931 \\
2 & 0,90533 & 0,93896 & 0,90555 & 19 & 0,96638 & 0,95711 & 0,97209 \\
3 & 0,93032 & 0,94205 & 0,92277 & 20 & 0,98093 & 0,96124 & 0,97904 \\
4 & 0,92245 & 0,94291 & 0,92701 & 21 & 0,98135 & 0,96606 & 0,98502 \\
5 & 0,92385 & 0,94343 & 0,92946 & 22 & 0,98837 & 0,96915 & 0,98795 \\
6 & 0,94066 & 0,94438 & 0,93374 & 23 & 0,98946 & 0,97302 & 0,99082 \\
7 & 0,94207 & 0,94549 & 0,93849 & 24 & 0,99152 & 0,97518 & 0,99211 \\
8 & 0,94232 & 0,94567 & 0,93919 & 25 & 0,99317 & 0,97518 & 0,99211 \\
9 & 0,93594 & 0,94575 & 0,93954 & 26 & 0,99283 & 0,98490 & 0,99603 \\
10 & 0,95231 & 0,94859 & 0,95004 & 27 & 0,99690 & 0,98851 & 0,99693 \\
11 & 0,95097 & 0,94928 & 0,95231 & 28 & 0,99863 & 0,99565 & 0,99815 \\
12 & 0,96110 & 0,95109 & 0,95781 & 29 & 0,99875 & 0,99608 & 0,99820 \\
13 & 0,95500 & 0,95109 & 0,95781 & 30 & 0,99879 & 0,99625 & 0,99822 \\
14 & 0,96231 & 0,95117 & 0,95806 & 31 & 0,99947 & $\mathbf{1 , 0 0 6 4 9}$ & 0,99914 \\
15 & 0,96562 & 0,95358 & 0,96442 & 32 & 0,99953 & $\mathbf{1 , 0 0 6 8 4}$ & 0,99916 \\
16 & 0,95705 & 0,95375 & 0,96484 & 33 & 0,99999 & $\mathbf{1 , 0 2 0 5 1}$ & 0,99968 \\
17 & 0,96739 & 0,95453 & 0,96666 & 34 & 0,99999 & $\mathbf{1 , 0 3 0 5 8}$ & 0,99984 \\
\hline \hline
\end{tabular}

ilustra uma desvantagem do uso do modelo de regressão normal linear, relativamente ao modelo de regressão beta, quando a variável resposta é medida de forma contínua no intervalo unitário. 


\section{Considerações Finais}

Nesse trabalho apresentamos o modelo de regressão beta proposto por Ferrari e Cribari-Neto (2004). Mais especificamente, apresentamos características e propriedades do modelo no que diz respeito a sua definição, funções de ligação, função escore, matriz de informação, estimação dos parâmetros de regressão, testes de hipóteses, intervalos de confiança e algumas medidas de diagnóstico. Uma característica desse modelo é o fato de que os parâmetros de regressão $\beta$ e o de precisão $\phi$ não são ortogonais, ao contrário do que é verificado na classe de modelos lineares generalizados.

Avaliamos através de simulação de Monte Carlo a qualidade das aproximações utilizadas nas inferências sobre os parâmetros do modelo de regressão beta em amostras finitas. Obtivemos para os estimadores de regressão excelentes resultados em relação a viés, comportamento assintótico (medido através dos coeficientes de assimetria e curtose), taxa de cobertura e assimetria dos intervalos de confiança, mesmo para tamanhos amostrais pequenos $(n=20)$. No entanto, para o parâmetro de precisão os resultados são menos satisfatórios: os estimadores apresentaram viés positivo e sua distribuição mostrout-se assimétrica à direita. Notamos também que, o parâmetro de precisão exerce influência apenas nas estimativas intervalares dos parâmetros de regressão, enquanto que as estimativas pontuais não foram afetadas. 
Pesquisamos também, os tamanhos dos testes da razão de verossimilhanças, escore e Wald. Das simulações, concluímos que o teste escore forneceu taxas de rejeição da. hipótese nula mais próximos dos respectivos níveis nominais.

Apresentamos também duas novas aplicações do modelo de regressão beta a conjuntos de dados reais. Nessas aplicações mostramos que o modelo de regressão beta surge como uma nova proposta para ajustar dados que têm seu limite de variação no intervalo unitário $(0,1)$. Evidenciamos que o ajuste da regressão normal linear pode fornecer valores preditos fora do intervalo unitário, sendo que o mesmo não ocorre no modelo de regressão beta.

Por fim, destacamos o fato de que não há dificuldades computacionais importantes para ajustar o modelo de regressão beta. O programa utilizado para as aplicações é apresentado (ver Apêndice C). 


\section{Informação de Fisher para $(p, q)$}

Neste apêndice obtemos a matriz de informação de Fisher para os parâmetros $(p, q)$ da distribuição beta. O logaritmo natural da função de verossimilhança para uma amostra $y_{1}, \ldots, y_{n}$ é dado por

$$
\ell(p, q)=-n \log B(p, q)+(p-1) \sum_{i=1}^{n} \log y_{i}+(q-1) \sum_{i=1}^{n} \log \left(1-y_{i}\right)
$$

Substituindo $B(p, q)$ dado em (2.4) na expressão anterior, encontramos

$$
\begin{aligned}
\ell(p, q)= & -n[\log \Gamma(p)+\log \Gamma(q)-\log \Gamma(p+q)] \\
& +(p-1) \sum_{i=1}^{n} \log y_{i}+(q-1) \sum_{i=1}^{n} \log \left(1-y_{i}\right) .
\end{aligned}
$$

As derivadas de $1^{\underline{a}}$ ordem de $\ell(p, q)$ com respeito a $p$ e $q$ são dadas por

$$
\frac{\partial \ell(p, q)}{\partial p}=-n[\psi(p)-\psi(p+q)]+\sum_{i=1}^{n} \log y_{i}
$$

e

$$
\frac{\partial \ell(p, q)}{\partial q}=-n[\psi(q)-\psi(p+q)]+\sum_{i=1}^{n} \log \left(1-y_{i}\right) .
$$

As derivadas de $2^{\underline{a}}$ ordem de $\ell(p, q)$ com respeito a cada parâmetro são

$$
\frac{\partial^{2} \ell(p, q)}{\partial p^{2}}=-n\left[\psi^{\prime}(p)-\psi^{\prime}(p+q)\right]
$$


$e$

$$
\frac{\partial^{2} \ell(p, q)}{\partial q^{2}}=-n\left[\psi^{\prime}(q)-\psi^{\prime}(p+q)\right]
$$

Finalmente, a derivada de $2^{\underline{a}}$ ordem de $\ell(p, q)$ com respeito a $p$ e $q$ pode ser escrita como

$$
\frac{\partial^{2} \ell(p, q)}{\partial p \partial q}=n \dot{\psi}^{\prime}(p+q)
$$

A partir de (A.1), (A.2) e (A.3), obtemos a matriz de informação de Fisher para $(p, q)$ dada por

$$
K(p, q)=-n\left(\begin{array}{cc}
\psi^{\prime}(p)-\psi^{\prime}(p+q) & -\psi^{\prime}(p+q) \\
-\psi^{\prime}(p+q) & \psi^{\prime}(q)-\psi^{\prime}(p+q)
\end{array}\right) .
$$

Agora, para obtenção de $K^{-1}(p, q)$ dada em (2.12), utilizam-se procedimentos usuais de inversa de matriz $2 \times 2$. 


\section{Matriz de Covariâncias}

\section{Assintóticas para os EMM}

Neste apêndice apresentamos os cálculos necessários para a obtenção da matriz de covariâncias assintóticas dos estimadores do método dos momentos. No que segue utilizamos resultados da Seção 5.4 do livro de Sen e Singer (1993), o qual apresenta propriedades assintóticas dos estimadores do método dos momentos.

Sejam $X_{1}, \ldots, X_{n}$ variáveis aleatórias independentes com função densidade $f(x ; \theta)$, $\theta \in \Theta \subset \mathbb{R}^{q}, q \geq 1, x \in \mathbb{R}$, e assuma que $\mathrm{E} X_{1}^{k}=\mu_{k}^{\prime}=h_{k}(\theta)<\infty, k=1, \ldots, q$; também, seja $m_{k n}^{\prime}=n^{-1} \sum_{i=1}^{n} X_{i}^{k}, k=1, \ldots, q$. O estimador do método dos momentos $\widetilde{\theta}$ de $\theta$ é uma solução (em $\theta$ ) para as equações $h_{k}(\theta)=m_{k n}^{\prime}, k=1, \ldots, q$. O teorema seguinte estabelece condições sob as quais a distribuição de $\widetilde{\theta}$ pode ser obtida.

Teorema: Considere a situação descrita acima. Assuma que $\mu_{k}^{\prime}<\infty, k=1, \ldots, 2 q$. Também, seja $h(\theta)=\left\{h_{1}(\theta), \ldots, h_{q}(\theta)\right]^{\top}$ e $H(\theta)=(\partial / \partial \theta) \mathrm{h}(\theta)$ de tal forma que o posto de $H(\theta)$ é q e seus elementos são denotados por $H_{i j}(\theta)=\left(\partial / \partial \theta_{j}\right) h_{i}(\theta)$, com 
$i, j=1, \ldots, q$, contínuos em $\theta$. Então,

$$
\sqrt{n}(\tilde{\theta}-\theta) \stackrel{\mathcal{D}}{\longrightarrow} \mathcal{N}_{q}\left(0,[H(\theta)]^{-1} \Sigma\left\{[H(\theta)]^{-1}\right\}^{\top}\right)
$$

$\operatorname{com} \sum$ sendo uma matriz $q \times q \operatorname{com}(j, k)$-ésimo elemento dado por $\mu_{j+k}^{\prime}-\mu_{j}^{\prime} \mu_{k}^{\prime}$.

A demonstração deste teorema encontra-se em Sen e Singer (1993, Seção 5.4). Podemos reescrever este resultado da seguinte forma

$$
\widetilde{\theta} \sim \mathcal{N}_{q}(\theta, A(\theta))
$$

aproximadamente, em que $A(\theta)=n^{-1}[H(\theta)]^{-1} \Sigma\left\{[H(\theta)]^{-1}\right\}^{\top}$, ou seja, a matriz de covariâncias assintóticas do estimador do método dos momentos. No caso particular da distribuição beta (ver Seção 2.2), temos que $\theta=(p, q)$ e $\widetilde{\theta}=(\widetilde{p}, \widetilde{q})$. Logo, o vetor $h(p, q)$ conterá os primeiros quatros momentos populacionais da distribuição $\operatorname{Beta}(p, q)$, sendo dado por

$$
h(p, q)=\left(\begin{array}{c}
h_{1}(p, q) \\
h_{2}(p, q) \\
h_{3}(p, q) \\
h_{4}(p, q)
\end{array}\right)^{\top}=\left(\begin{array}{c}
\frac{p}{p+q} \\
\frac{p(p+1)}{(p+q)(p+q+1)} \\
\frac{p(p+1)(p+2)}{(p+q)(p+q+1)(p+q+2)} \\
\frac{p(p+1)(p+2)(p+3)}{(p+q)(p+q+1)(p+q+2)(p+q+3)}
\end{array}\right)^{\top} .
$$

A matriz $H(p, q)$, de dimensão $2 \times 2$, que contém a derivada de $1^{\underline{a}}$ ordem dos dois primeiros momentos populacionais em relação a cada parâmetro fica dada por

$$
H(p, q)=\left(\begin{array}{cc}
\frac{\partial h_{1}(p, q)}{\partial p} & \frac{\partial h_{1}(p, q)}{\partial q} \\
\frac{\partial h_{2}(p, q)}{\partial p} & \frac{\partial h_{2}(p, q)}{\partial q}
\end{array}\right)=\left(\begin{array}{cc}
\frac{q}{(p+q)^{2}} & -\frac{p}{(p+q)^{2}} \\
\frac{q\left(2 p^{2}+2 p q+2 p+q+1\right)}{(p+q)^{2}(p+q+1)^{2}} & -\frac{p(p+1)(2 p+2 q+1)}{(p+q)^{2}(p+q+1)^{2}}
\end{array}\right)
$$

A inversa de $H(p, q)$ é dada por

$$
[H(p, q)]^{-1}=\left(\begin{array}{cc}
\frac{(p+1)(2 p+2 q+1)(p+q)}{q} & -\frac{(p+q)(p+q+1)^{2}}{q} \\
\frac{\left(2 p^{2}+2 p q+2 p+q+1\right)(p+q)}{p} & -\frac{(p+q)(p+q+1)^{2}}{p}
\end{array}\right) .
$$


Finalmente, a matriz $\Sigma$, de dimensão $2 \times 2$, é dada por

$$
\Sigma=\left(\begin{array}{cc}
\frac{p+q}{(p+q)^{2}(p+q+1)} & \frac{2 p q(p+1)}{(p+q)^{2}(p+q+1)(p+q+2)} \\
\frac{2 p q(p+1)}{(p+q)^{2}(p+q+1)(p+q+2)} & \frac{2 p q(p+1)\left(2 p^{2}+2 p q+6 p+3 q+3\right)}{(p+q)^{2}(p+q+1)^{2}(p+q+2)(p+q+3)}
\end{array}\right) .
$$

A partir de (B.1), (B.2) e (B.3), fica fácil obter a matriz de covariâncias assintóticas dos estimadores do métodos dos momentos $(A(p, q))$, dada em (2.14). 
Apêndice C

\section{Programas}

Apresentamos neste apêndice dois programas que foram desenvolvidos na linguagem de programação 0x. O primeiro é o de simulação que foi utilizado no Capítulo 4 para avaliação de resultados assintóticos em amostras finitas. O segundo programa foi utilizado para a análise do conjunto de dados do exemplo de Oxidação de Amônia apresentado no Capítulo 5. O programa utilizado na outra aplicação é aqui omitido, devido à grande semelhança com este último programa. Os dois programas foram desenvolvidos baseados no programa que foi construído por Ferrari e Cribari-Neto (2003), que está disponível em http://www.de.ufpe.br/ cribari/ betareg_example.zip. Os programas desta dissertação também estão disponíveis em http://www. ime.usp.br/ mso.

\section{Programa de Simulação}

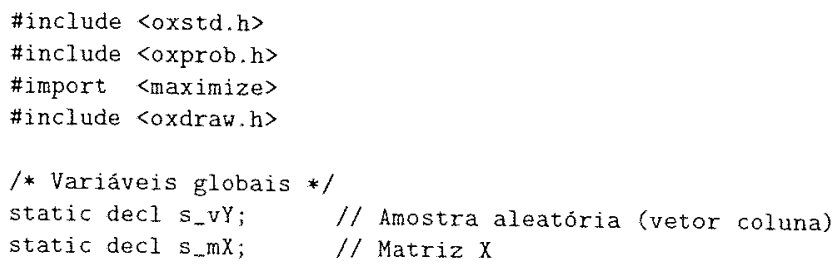




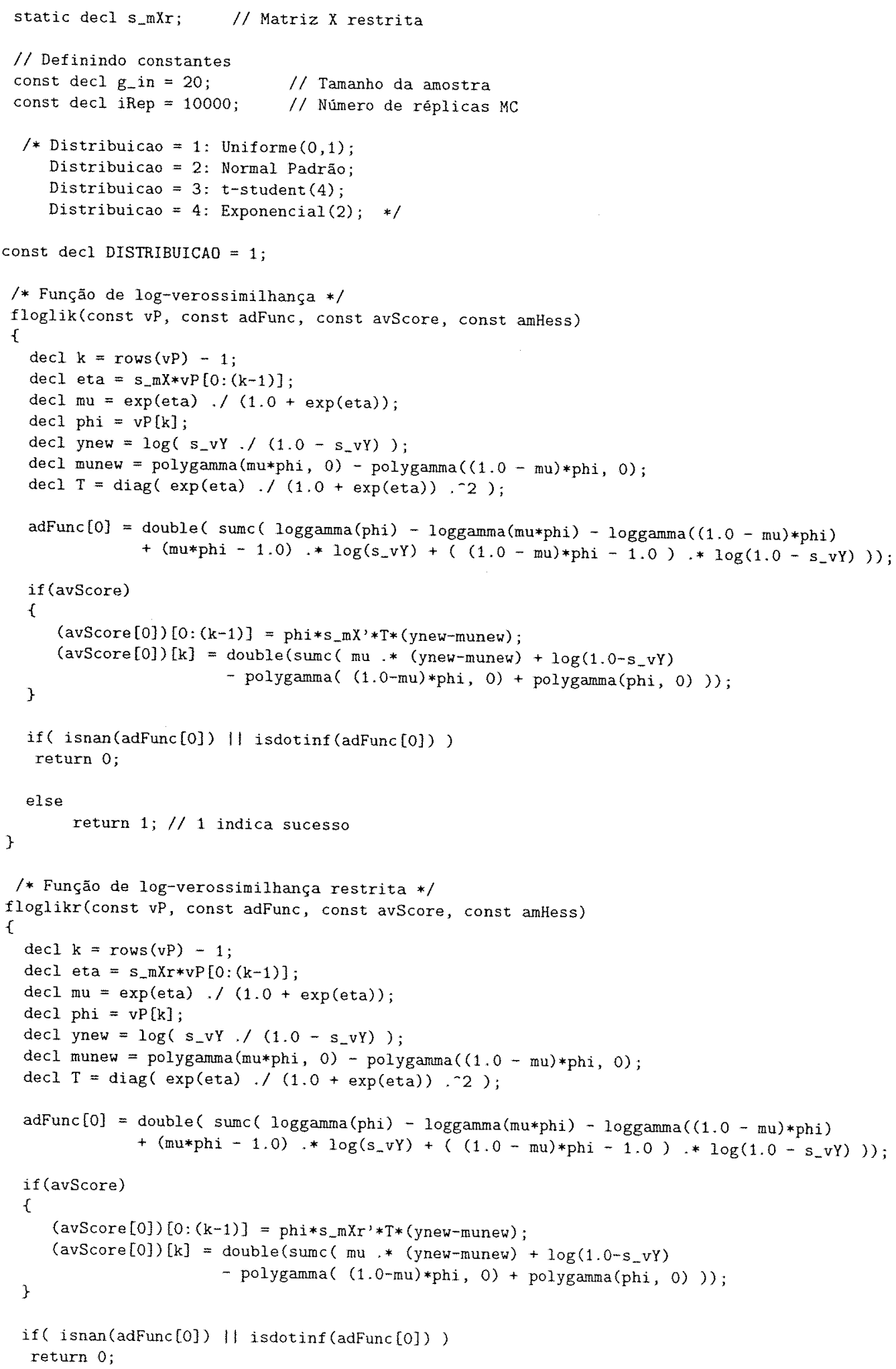




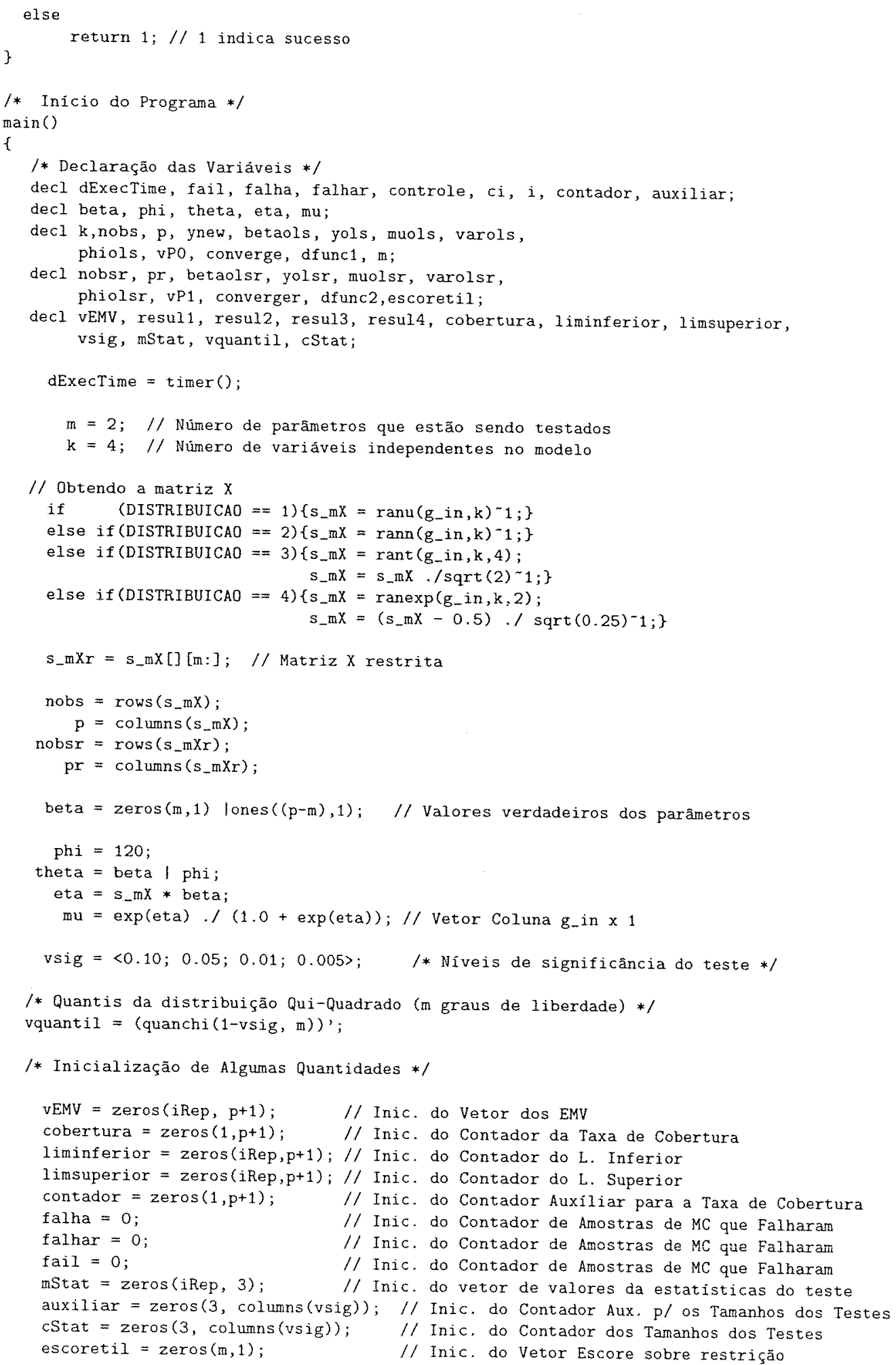




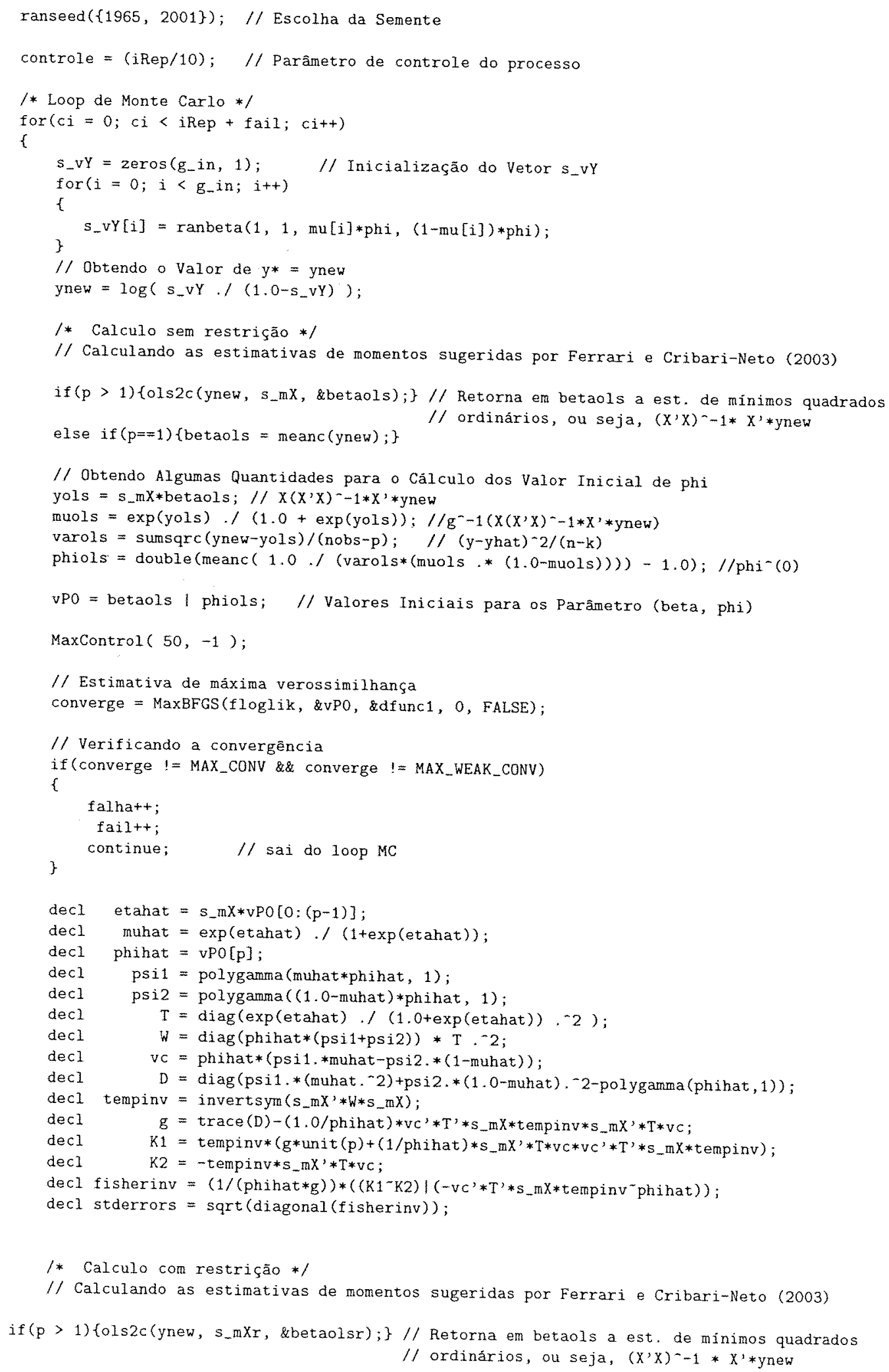




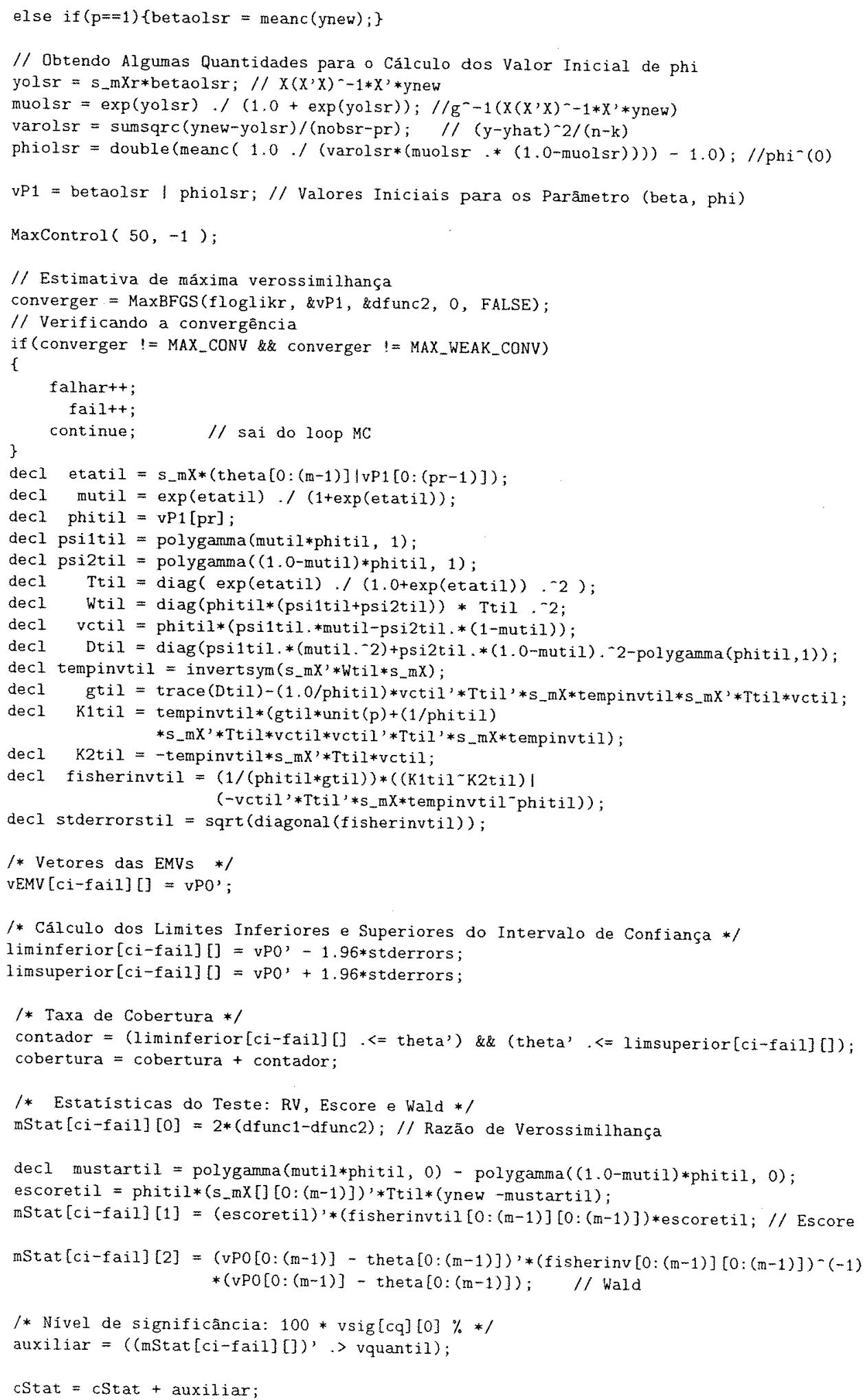




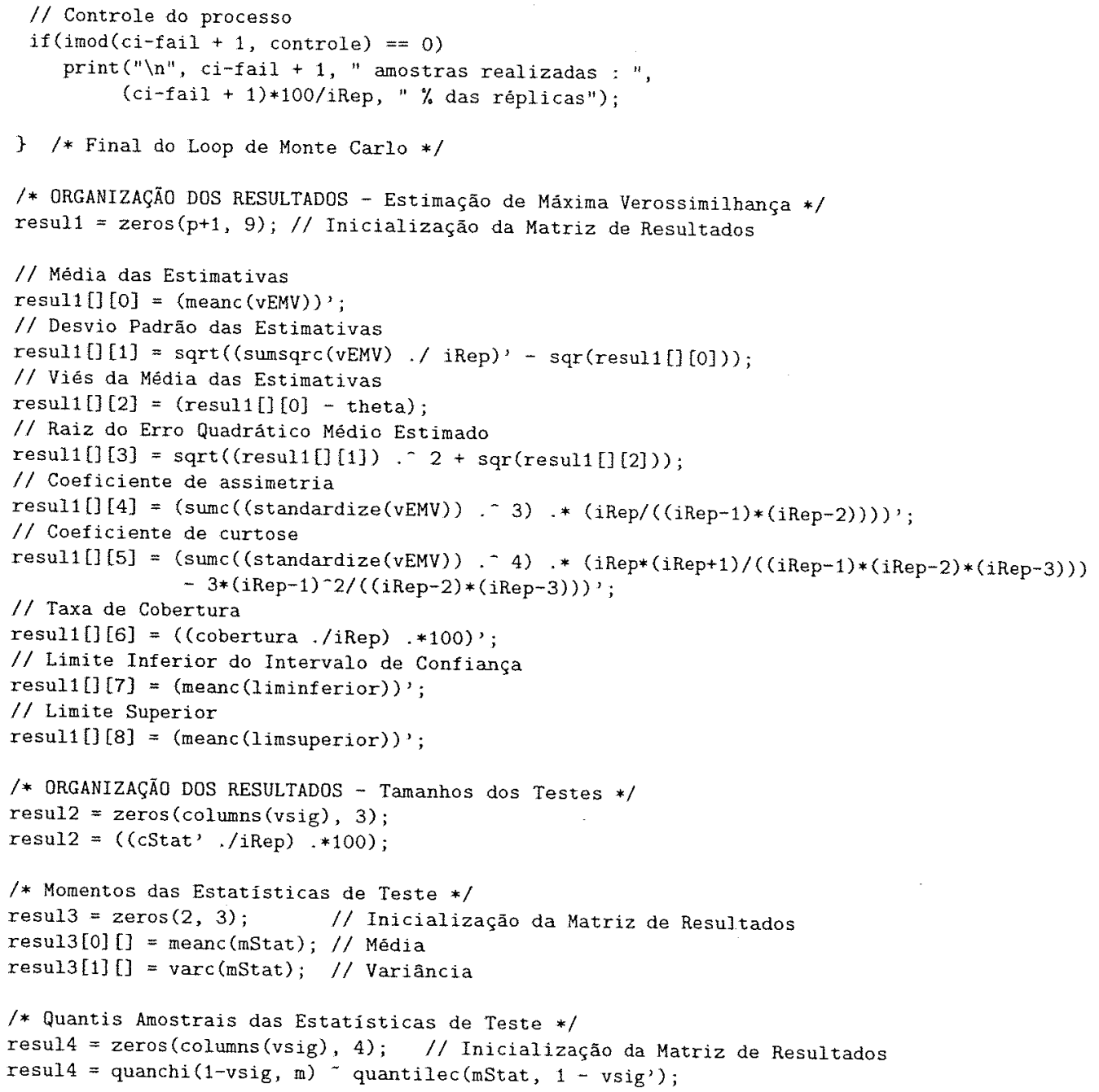




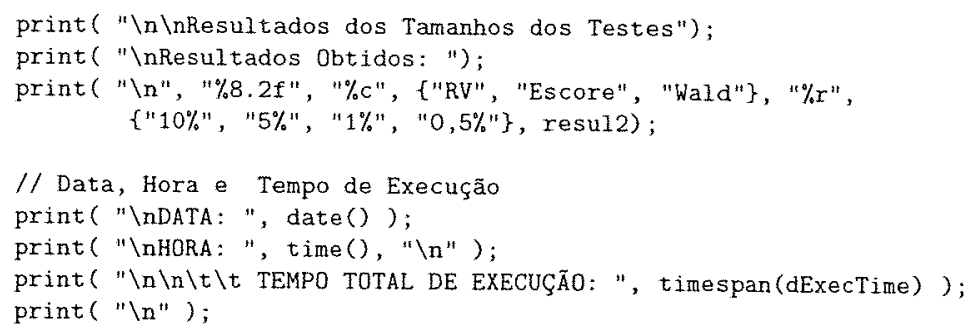

\section{Exemplo: Oxidação de Amônia}

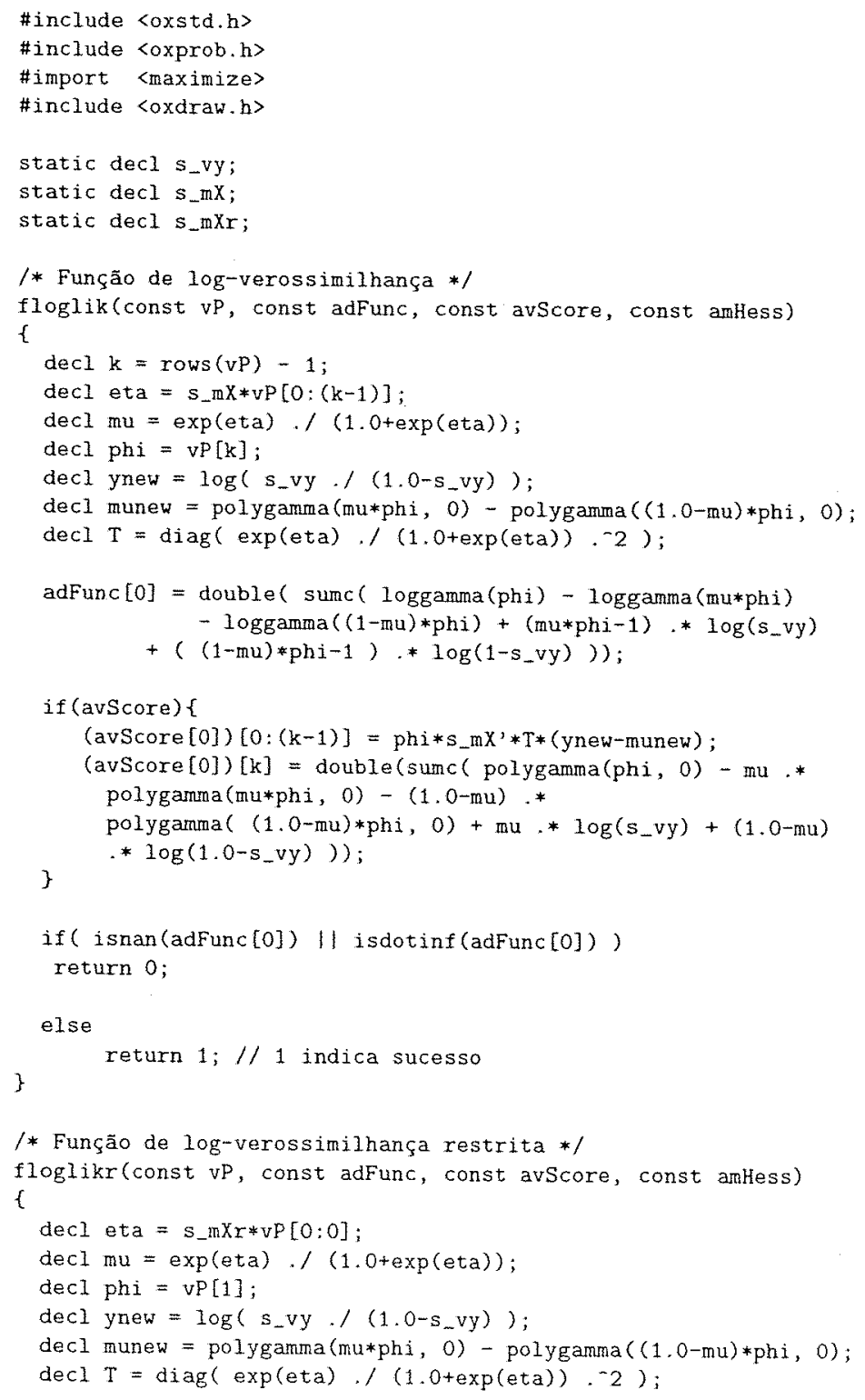




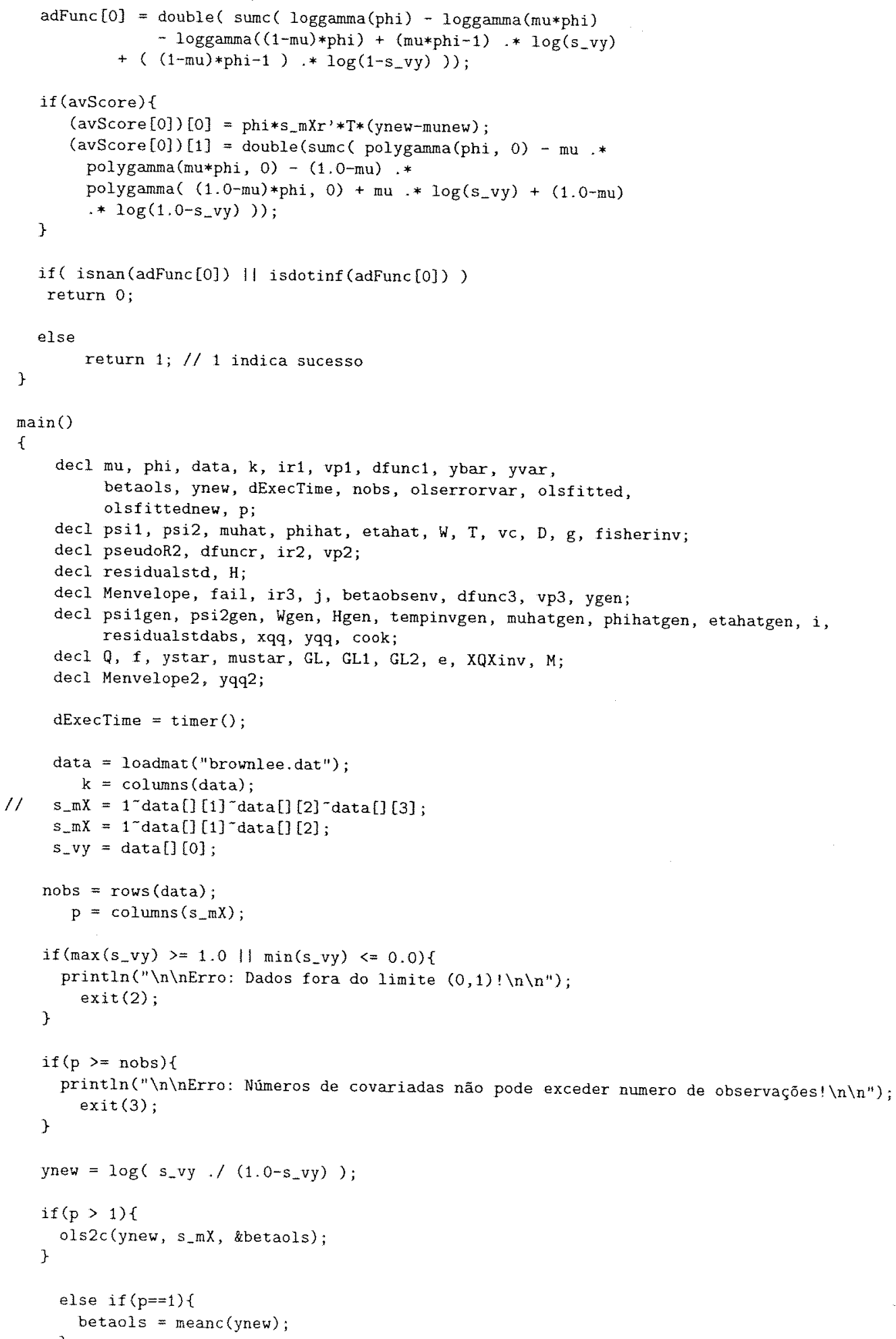




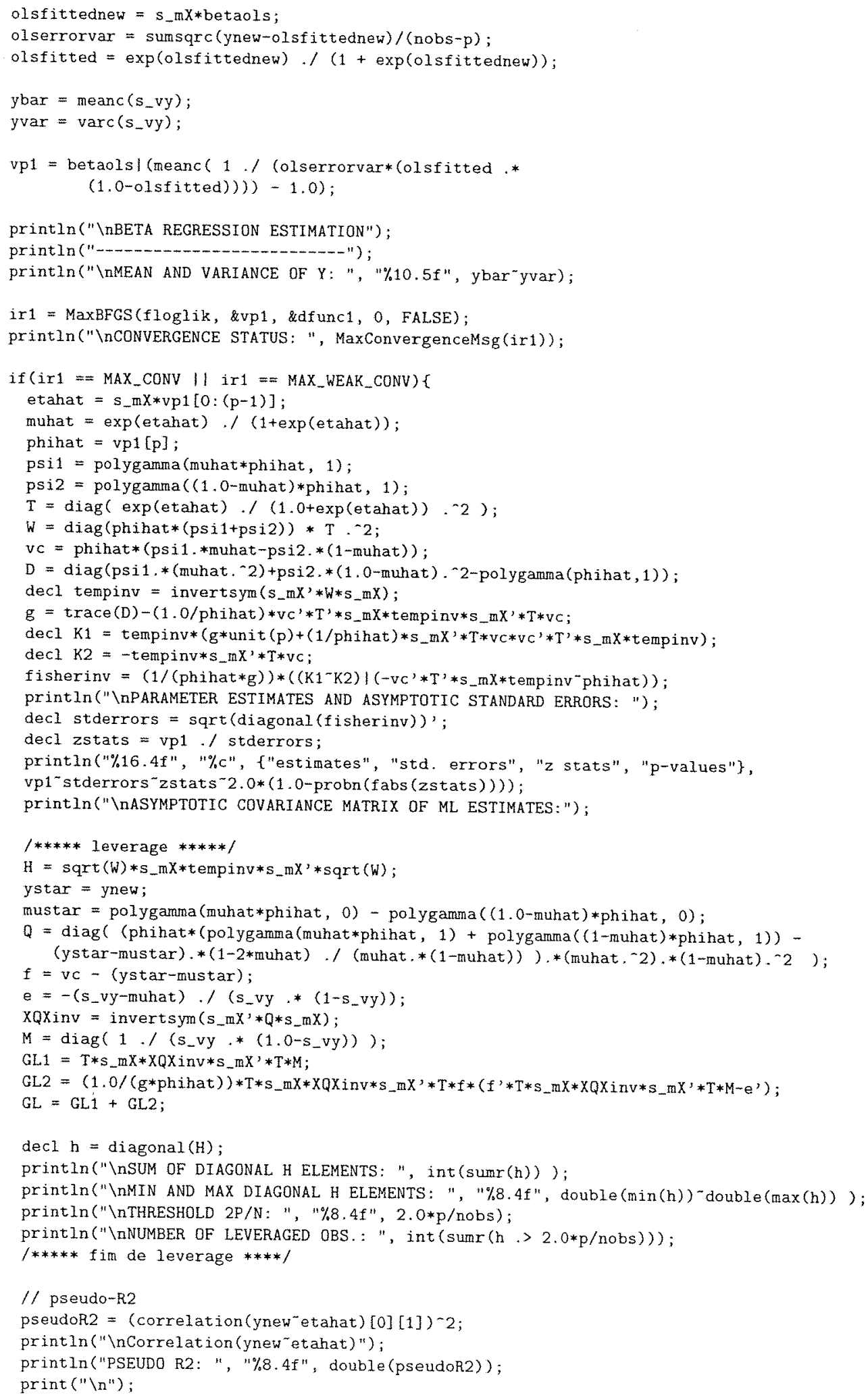


// R2 calculado como corr (y, g-1 (etahat))

decl pseudoR2star $=\left(\text { correlation }\left(s_{-} \text {vy muhat }\right)[0][1]\right)^{-2}$;

print ("Correlation (s_vy"muhat)");

println("\nPSEUDO R2 star: ", "\%8.4f", double(pseudoR2star));

// R2 baseado em log-verossimilhança

$s_{-} \mathrm{mXr}=$ ones (nobs, 1 );

decl ynewbar $=$ meanc $($ ynew $)$;

decl muhatr $=\exp (y n e w b a r) /(1,0+\exp ($ ynewbar $))$

$v_{p} 2=$ yrewbarl $(1.0 /(\operatorname{varc}($ ynew $) *$ muhatr $*(1.0$-muhatr $))-1.0)$

$/ /$ restricted maximum likelihood estimation

ir2 $=\operatorname{MaxBFGS}(f \log l i k r, \& v p 2$, \&dfuncr, 0 , FALSE);

if $($ ir $2=m$ MAX_CONV $1 \mid$ MAX_WEAK_CONV) (

decl pseudoR2LR $=1.0-(\exp (\operatorname{dfuncr}) / \exp (\operatorname{dfunc1}))^{-}(2 /$ nobs $)$;

println("\nPSEUDO R2 LR (1 - (L(0)/L(betahat)) - (2/n)): ", "\%8.4f", double (pseudoR2LR));

residualstd $=\operatorname{sqrt}(1.0+$ phihat $) *\left(s_{-} v y\right.$-muhat $) . /$ sqrt (muhat. $*(1-$ muhat $\left.)\right) ;$

decl ell1 = loggamma (phihat) - loggamna (s_vy*phihat) - loggamma( (1-s_vy)*phihat)

$+\left(s_{-} v y *\right.$ phihat-1) .* $\log \left(s_{-} v y\right)+\left(\left(1-s_{-} v y\right) *\right.$ phihat-1 $) . * \log \left(1-s_{-} v y\right)$

decl el12 = loggamma (phihat) - loggamma (muhat*phihat) - loggamma( (1-muhat)*phihat)

$+\left(\right.$ muhat*phihat-1) $* \log \left(s_{-} v y\right)+((1-$ muhat $) *$ phihat -1$) * \log \left(1-s_{-} v y\right)$;

decl mysign $=\left(s_{-}\right.$vy - muhat),$>0 . ? 1.0 .:-1.0$;

decl residualdev $=$ mysign.$* \operatorname{sqrt}(2.0 *(e l 11-\mathrm{e} 112))$;

cook $=\left(h^{\prime}, *\right.$ residualstd. $\left.{ }^{2}\right) . /\left(p^{*}\left(1-h^{\prime}\right) * * 2\right)$;

Menvelope $=$ zeros(nobs, 19);

Menvelope2 $=\operatorname{zeros}($ nobs, 19$)$;

fail $=0$;

for $(j=0 ; j<19 ; j++)\{$

ygen $=$ zeros (nobs, 1$)$;

for $(i=0 ; i<$ nobs; $i++)$ \}

ygen $[i]=\operatorname{ranbeta}(1,1$, muhat $[i] *$ phihat, (1-muhat [i] $) *$ phihat);

$v p 3=v p 1$

psilgen = polyganna (muhat*phihat, 1$)$;

psi2gen = polygama $((1.0$-muhat $) *$ phihat, 1$)$;

Wgen $=\operatorname{diag}($ phihat* $($ psi1+psi2) $) * \mathrm{~T} .-2$

tempinvgen $=$ invertsym $\left(s_{-} m X *\right.$ Wgen*s_mX);

Hgen $=\operatorname{sqrt}($ Wgen $) * s_{-} m X *$ tempinvgen*s_m $X^{\prime} *$ sqrt $($ Wgen $)$

etahatgen $=s_{-} \mathrm{mX} * \mathrm{vp} 3[0:(\mathrm{p}-1)]$;

muhatgen $=\exp ($ etahatgen $) \cdot /(1.0+\exp ($ etahatgen $)) ;$

phihatgen $=v p 3[p]$.

Menvelope []$[j]=\operatorname{sqrt}(1.0+$ phihatgen $) *(y g e n-m u h a t g e n) . /$ sqrt $($ muhatgen.*(1-muhatgen $))$;

decl ell1gen = loggamma (phihat) - loggamma (ygen*phihat) - loggamma ( 1 -ygen $) *$ phihat $)$

$+($ ygen*phihat-1) * $* \log ($ ygen $)+((1-$ ygen $) *$ phihat -1$) * * \log (1-y g e n)$;

decl ell2gen = logganma (phihat) - loggamma (muhat*phihat $)-\operatorname{loggamma}((1-$ muhat $) *$ phihat $)$

$+($ muhat*phihat -1$) * \log ($ ygen $)+((1-$ muhat $) *$ phihat -1$) * \log (1-y g e n)$

decl mysigngen $=($ ygen - muhat $) .>0 . ? 1.0 .:-1.0$;

decl residualdev $=$ mysigngen $* * \operatorname{sqrt}(2.0 *(e 111$ gen-ell2gen $))$;

Menvelope2[] $[j]=$ residualdev

\}

residualstdabs $=$ fabs (residualstd);

Menvelope $=\operatorname{sortc}($ fabs (Menvelope) $)$

$\mathrm{xqq}=$ quann $\left(\right.$ (range $(1, \text { nobs })^{\prime}+$ nobs $\left.-1 / 8\right) /(2 *$ nobs $\left.+0,5)\right) ;$

yqq $=\operatorname{zeros}($ nobs, 4$)$;

yqq[][3] $=\operatorname{sortc}($ residualstdabs $)$;

yqq []$[0]=\operatorname{minc}($ Menvelope')';

yqq[] [1] = meanr (Menvelope);

yqq[] [2] = $\operatorname{maxc}($ Menvelope')';

decl residualdevabs $=$ fabs (residualstd);

Menvelope $2=\operatorname{sortc}($ fabs (Menvelope 2$)$ ); 


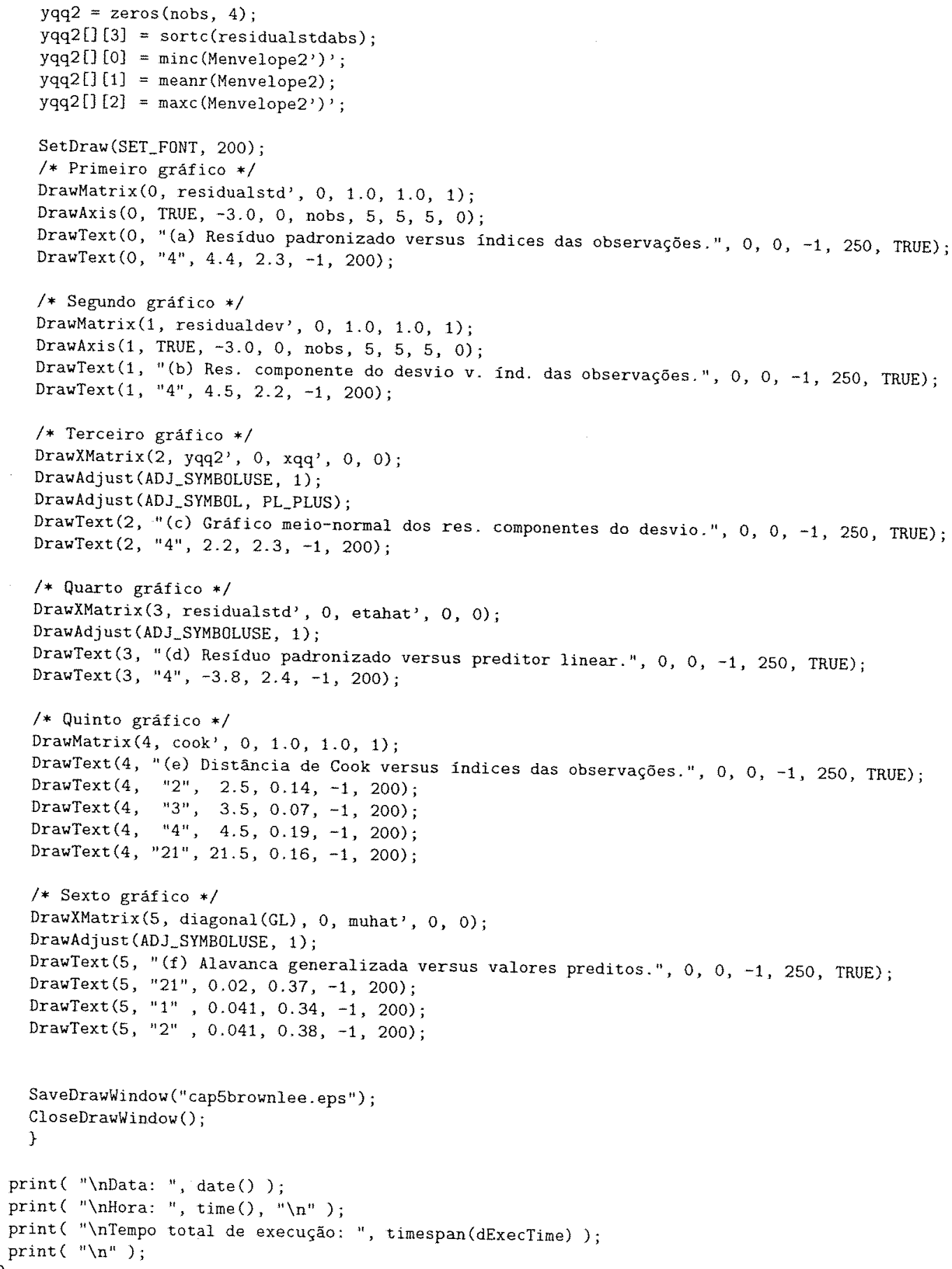




\section{Referências Bibliográficas}

[1] Abramowitz, M. e Stegun, I. A. (1965). Handbook of Mathematical Functions with Formulas, Graphs and Mathematical Tables. New York: Dover.

[2] Atkinson, A. C. (1985). Plots, Transformations and Regression: An Introduction to Graphical Methods of Diagnostic Regression Analysis. New York: Oxford University Press.

[3] Barifouse, R. (2004). O Amendoim sob suspeita. Revista Ciência Hoje 34, No $201,48-49$.

[4] Brownlee, K. A. (1965). Statistical Theory and Methodology in Science and Engineering. $2^{\circ}$ edição. London: John Wiley \& Sons.

[5] Bury, K. (1999). Statistical Distributions in Engineering. New York: Cambridge University Press.

[6] Chatterjee, S. e Hadi, A. S. (1986). Influential observations, high leverage points, and outliers in linear regression (with discussion). Statistical Science 1, $379-416$.

[7] Cook, R. D. (1977). Detection of infuential observations in linear regressions. Technometrics 19, 15-18. 
[8] Cook, R. D. (1979). Influential observation in linear regression. Journal of the American Statistical Association 74, 169-174.

[9] Cook, R. D. e Weisberg, S. (1982). Residuals and Influence in Regression. New York: Chapman and Hall.

[10] Cordeiro, G. (1992). Introduçâao à Teoria de Verossimilhança. Livro texto de minicurso, $10^{\circ}$ Simpósio Nacional de Probabilidade e Estatística, UFRJ, Rio de Janeiro.

[11] Costa Neto, P. L. O. (1977). Estatística. São Paulo: Edgard Blücher.

[12] Cox, D. R. e Snell, E. J. (1968). A general definition of residuals (with discussion). Journal of the Royal Statistical Society B 30, 248-275.

[13] Daniel, C. e Wood, F. S. (1971). Fitting Equations To Data. New York: John Wiley.

[14] Doornik, J. A. (2001). Ox: An Object-oriented Matrix Programming Language. $4^{\circ}$ edição. London: Timberlake Consultants e Oxford: http://www.nuff. ox.ac.uk/Users/Doornik.

[15] Draper, N. R. e Smith, H. (1966). Applied Regression Analysis. New York: Wiley.

[16] Draper, N. R. e Smith, H. (1998). Applied Regression Analysis. $3^{\circ}$ edição. New York: Wiley.

[17] Ferrari, S. L. P. e Cribari-Neto, F. (2004). Beta regression for modeling rates and proportions. Journal of Applied Statistics, a aparecer.

[18] Fonseca, H. (2004). O Amendoim e a Aflatoxina. Disponível em http://www.micotoxinas. com.br/Boletim13.htm.

[19] Galea, M., Paula, G. A. e Uribe-Opazo, M. (2003). On influence diagnostics in univariate elliptical linear regression models. Statistical Papers 44, 23-45. 
[20] Johnson, N. L., Kotz, S. e Balakrishnan, N. (1995). Continuous Univariate Distributions, vol. 2. $2^{\circ}$ edição. New York: Wiley.

[21] McCullagh, P. e Nelder, J. A. (1989). Generalized Linear Models. $2^{\circ}$ edição. London: Chapman and Hall.

[22] Mittlböck, M. e Schemper, M. (1996). Explained Variation for Logistic Regression. Statistics in Medicine 15, 1987-1997.

[23] Nelder, J. A., Wedderburn, R. W. M. (1972). Generalized Linear Models. Journal of the Royal Statistical Society A 135, 370-384.

[24] Neter, J., Kutner, M. H., Nachtsheim, C. J. e Wasserman, W. (1996). Applied Linear Statistical Models. $4^{\circ}$ edição, Chicago: Irwin.

[25] Nocedal, J. e Wright, S. J. (1999). Numerical Optimization. New York: Springer-Verlag.

[26] Paula, G. A. (2003). Modelos de Regressão com Apoio Computacional. São Paulo: IME/USP. Disponivel em http://www.ime.usp.br/ giapaula.

[27] Pearson, K. (1895). Mathematical Contribution to the Theory of Evolution. II. Skew Variation in Homogeneous Material. Philosophical Transactions of the Royal Society of London, A 186, 343-414.

[28] Pearson, E. S. e Johnson, N. L. (1968). Tables of Incomplete Beta Function. Cambridge: Cambridge University Press.

[29] Phan-Gia, T. (1994). Value of the beta prior information. Communications in Statistics - Theory and Methods 23, 2175-2195.

[30] Rao, C. R. (1973). Linear Statistical Inference and Its Applications. $2^{\circ}$ edição. New York: Wiley.

[31] Sen, P. K. e Singer, J. M. (1993). Large Sample Methods in Statistics: An Introduction With Applications. New York: Chapman and Hall. 
[32] Wei, B.-C. Hu, Y.-Q. e Fung, W.-K. (1998). Generalized leverage and its applications. Scandinavian Journal of Statistics 25, 25-37. 